\title{
Multiple motors cooperate to establish and maintain acentrosomal spindle bipolarity in C. elegans oocyte meiosis
}

Gabriel Cavin-Meza, Michelle M. Kwan, and Sarah M. Wignall

Department of Molecular Biosciences, Northwestern University, Evanston, IL 60208

To whom correspondence should be addressed:

Sarah M. Wignall

Department of Molecular Biosciences

Northwestern University

2205 Tech Drive, Hogan 2-100

Evanston, IL 60208-3500

phone: 847-467-0386

fax: $847-491-4970$

s-wignall@northwestern.edu 


\section{ABSTRACT}

While centrosomes organize spindle poles during mitosis, oocyte meiosis can occur in their absence. Spindles in human oocytes frequently fail to maintain bipolarity and consequently undergo chromosome segregation errors, making it important to understand mechanisms that promote acentrosomal spindle stability. To this end, we have optimized the auxin-inducible degron system in C. elegans to remove factors from pre-formed oocyte spindles within minutes and assess effects on spindle structure. This approach revealed that dynein is required to maintain the integrity of acentrosomal poles; removal of dynein from bipolar spindles caused

9 pole splaying, and when coupled with a monopolar spindle induced by depletion of kinesin-12

10 motor KLP-18, dynein depletion led to a complete dissolution of the monopole. Surprisingly, we

11 went on to discover that following monopole disruption, individual chromosomes were able to reorganize local microtubules and re-establish a miniature bipolar spindle that mediated chromosome segregation. This revealed the existence of redundant microtubule sorting forces

14 that are undetectable when KLP-18 and dynein are active. We found that the kinesin-5 family motor BMK-1 provides this force, uncovering the first evidence that kinesin-5 contributes to $C$. elegans meiotic spindle organization. Altogether, our studies have revealed how multiple motors

17 are working synchronously to establish and maintain bipolarity in the absence of centrosomes. 


\section{INTRODUCTION}

Centrosomes serve as prominent microtubule organizing centers (MTOCs) during mitosis and provide a clear structural blueprint for the formation of a bipolar spindle. However, oocytes of many species lack centrosomes. In human oocytes, it has been shown that acentrosomal spindles are highly unstable; poles go through an extended period where they can split apart and then come back together, and spindles that display this instability have a high incidence of chromosome segregation errors (Holubcova et al., 2015). The causes of this instability and the mechanisms by which acentrosomal spindles are stabilized in the absence of centrosomes remain poorly understood.

C. elegans is an ideal model to investigate these questions, given similarities between worm and human oocytes. Specifically, while acentrosomal spindle assembly in some organisms relies on discrete microtubule organizing centers that help organize spindle poles (Schuh and Ellenberg, 2007), C. elegans and human oocytes do not appear to contain these structures (Holubcova et al., 2015; Wolff et al., 2016). In C. elegans, microtubules are instead nucleated in proximity to the disassembling nuclear envelope in a spherical cage-like structure. Microtubule minus ends are then pushed outwards to the periphery of the array by the kinesin12 family motor KLP-18, where they form multiple poles with clearly defined clusters of minus ends. Spindle poles then coalesce, eventually forming a bipolar structure with aligned chromosomes (Wolff et al., 2016; Gigant et al., 2017). Shortly after establishing a metaphase plate, the spindle shortens and rotates perpendicular to the cortex and chromosomes begin to segregate in anaphase (Albertson and Thomson, 1993). The cortical set of homologous chromosomes are discarded as a polar body, and the remaining sister chromatids undergo a second round of meiosis to generate the final set of maternal DNA.

While these stages of acentrosomal spindle assembly have been documented in multiple studies, the mechanisms by which microtubules organize into stable acentrosomal poles in this organism are not well understood. It would stand to reason that mitotic pole 
proteins are ideal candidates for also promoting meiotic pole stability; many proteins that serve important functions in C. elegans mitotic spindle assembly can be found within acentrosomal meiotic spindles (reviewed in (Severson et al., 2016; Mullen et al., 2019)). Various microtubuleassociated proteins (MAPs) and microtubule motors have been demonstrated to concentrate at poles and contribute to acentrosomal spindle assembly, such as ASPM-1 (Connolly et al., 2014), KLP-7 ${ }^{\text {MCAK }}$ (Connolly et al., 2015; Han et al., 2015; Gigant et al., 2017) and MEI-1/2 ${ }^{\text {katanin }}$ (Srayko et al., 2000; McNally et al., 2014). A study using a fast-acting temperature-sensitive mei-1 mutant demonstrated that katanin is essential to maintain spindle structure (McNally et al., 2014), but whether any other pole associated-proteins are required for spindle maintenance is not known.

Another factor that has been implicated in pole function is the minus-end directed microtubule motor dynein. Studies in other systems have shown that dynein is required for mitotic spindle pole focusing (reviewed in (Borgal and Wakefield, 2018)) and promoting poleward flux of newly formed microtubule minus ends (Elting et al., 2014; Hueschen et al., 2017). In C. elegans embryos, cortically-associated dynein is crucial for the proper positioning of both mitotic and acentrosomal meiotic spindles (Ellefson and McNally, 2009; van der Voet et al., 2009; Ellefson and McNally, 2011; Crowder et al., 2015). Moreover, a number of studies have shown that depletion of dynein or its cofactor dynactin causes spindle defects in oocytes, such as longer spindles with pole defects (Yang et al., 2005; Ellefson and McNally, 2009; Ellefson and McNally, 2011; Crowder et al., 2015; Muscat et al., 2015). However, since dynein is essential for development, these prior studies relied on either partial depletion or on temperature-sensitive mutants, which might not be completely null, so the effect of complete dynein inhibition on spindle assembly has not been assessed. Moreover, whether dynein is required to maintain acentrosomal pole integrity has not been tested. Thus, there is a need for an approach that can completely remove dynein function from oocytes in a conditional manner. 
Here, we have addressed this challenge using the auxin-inducible-degron (AID) system

70 (Zhang et al., 2015). We found that this method could efficiently remove dynein from oocytes

71 within minutes, enabling us to remove this protein either prior to spindle assembly or after

72 bipolarity has already been established. This approach revealed that dynein is essential for both

73 the formation and stabilization of acentrosomal spindle poles. Removing dynein from monopolar

74 spindles caused catastrophic breakdown of the monopole, further supporting a role for dynein in

75 pole integrity. Moreover, this experiment also revealed a striking phenotype that led to additional

76 insights into acentrosomal spindle function. Specifically, we found that following disruption of the

77 monopole, individual chromosomes were able to reorganize local microtubule bundles into a

78 bipolar spindle that could mediate anaphase-like segregation. This phenotype provided an

79 opportunity to identify proteins contributing to spindle bipolarity that are masked under normal

80 conditions. Through this assay, we identified kinesin- 5 motor BMK-1 as an outward sorting force

81 on microtubules, providing the first evidence that kinesin-5 contributes to spindle assembly in $C$. elegans. Altogether, these studies have furthered our understanding of the motor forces that contribute to acentrosomal spindle assembly and maintenance during meiosis. 


\section{RESULTS}

\section{Dynein is localized to acentrosomal spindles and is required for pole coalescence}

Since dynein is required for multiple cellular processes in C. elegans, previous studies using RNAi have used partial depletion to avoid impacting worm development (Yang et al., 2005; Ellefson and McNally, 2009; Ellefson and McNally, 2011; Muscat et al., 2015; McNally et al., 2016; Laband et al., 2017). In an attempt to achieve more complete depletion, we utilized a strain in which the heavy chain of dynein, DHC-1, was tagged at the endogenous locus with both GFP and a degron tag. This strain also expressed the ubiquitin ligase TIR1 using the sun-1 promotor to enable auxin-inducible degradation (AID) of DHC-1 specifically in the germ line (Zhang et al., 2015) (Figure 1A; we refer to this strain as "Dynein AID”). With this system, we are able to deplete dynein on a shorter time scale than RNAi, allowing us to assess the effects of full dynein depletion on spindle assembly and stability without affecting prior processes. To validate that this strain worked for efficient DHC-1 depletion, we imaged one-cell stage mitotic embryos using immunofluorescence (IF) and looked for canonical dynein depletion phenotypes, such as failure to separate centrosomes and improper mitotic spindle positioning (Gonczy et al., 1999; Nguyen-Ngoc et al., 2007; Kiyomitsu and Cheeseman, 2013). After a 4-hour incubation of adult Dynein AID worms on auxin-containing plates, we observed clear defects in centrosome separation and spindle positioning along the A-P axis and dynein was undetectable (Figure 1B), confirming strong depletion in embryos under conditions where germline and worm development are not affected.

To further validate the Dynein AID strain, we assessed the localization of tagged dynein throughout oocyte meiosis and found that the pattern was consistent with previous studies

(Figure 1C) (Ellefson and McNally, 2009; van der Voet et al., 2009; Ellefson and McNally, 2011; Crowder et al., 2015). Dynein was weakly associated with forming and bipolar spindles with a slight enrichment at acentrosomal poles labeled by the microtubule minus marker ASPM-1, and 
then became strongly enriched at poles during early anaphase; this enrichment decreased by late anaphase, with a population being retained near the cortex. Live imaging corroborated our fixed imaging (Video 1) and also revealed a population of dynein at kinetochores, as has been shown in other live imaging studies (McNally et al., 2016; Danlasky et al., 2020).

Given this initial validation, we created a Dynein AID strain that also expressed GFP::tubulin and GFP::histone and then assessed the effects of dynein depletion in oocytes by quantifying spindle morphology in intact worms (Figure 1D). The C. elegans germ line is arranged in a production-line fashion. Oocytes are fertilized in the -1 position of the germ line, and then spindles assemble as the newly fertilized embryo moves through the spermatheca and into the +1 position (Wolff et al., 2016). Under control conditions, bipolar spindles with focused poles were prevalent by the time the embryo reached the +1 position (Figure 1D). However, following 4 hours of auxin treatment, spindles mostly had unfocused poles; a large number of spindles displayed microtubule bundles arranged in a relatively parallel array, similar to what was observed following depletion of dynactin component DNC-1 in a previous study (Crowder et al., 2015). Imaging of ASPM-1 confirmed this phenotype; following 4-hour auxin treatment, microtubule minus ends lacked coalesced points between the ends of microtubule bundles (Figure 1E, 1F). Moreover, we also saw defects in spindle rotation in anaphase, consistent with previous studies (Ellefson and McNally, 2009; van der Voet et al., 2009; Ellefson and McNally, 2011; Crowder et al., 2015). These findings confirm the prediction that dynein is required for pole focusing during spindle assembly in oocytes, and validate the use of the Dynein AID strain to study dynein in oocytes and embryos.

\section{Dynein is required throughout meiosis to maintain focused poles}

After corroborating that dynein was required to focus acentrosomal poles during spindle assembly, we next sought to determine whether dynein was required to maintain focused poles. We therefore arrested oocytes in Metaphase I to enrich for pre-formed spindles (by depleting 
anaphase-promoting complex component EMB-30 (Furuta et al., 2000)) and then soaked worms in auxin for 25-30 minutes to acutely deplete dynein. Under these conditions, nearly all spindles had severely splayed poles (Figure 2A, 2B); microtubule bundles ran laterally alongside the bivalents, but the ends of the bundles were not brought together into focused poles. While ASPM-1 was enriched at these poles, it also was more dispersed throughout the spindle, suggesting a decrease in poleward transport of microtubule minus ends that has been reported in dynein-depleted mitotic spindles (Hueschen et al., 2017). Moreover, spindles appeared significantly longer after acute dynein depletion (Figure 2 - figure supplement 1A). We also observed identical spindle phenotypes in the absence of the metaphase arrest (Figure

\section{A, 2B, Figure 2 - figure supplement 1B).}

To confirm these results and to assess the dynamics of pole splaying, we turned to live ex utero imaging of embryos to film acute dynein AID depletion from metaphase-arrested spindles. We generated a Dynein AID strain expressing mCherry::tubulin so that we could visualize the spindle while dynein, tagged with GFP, was depleted. In the absence of auxin, there was no noticeable change in dynein localization or brightness throughout the imaging timeframe (Figure 2C, Video 2). In contrast, when embryos were dissected into auxin, dynein appeared depleted from meiotic spindles within roughly three minutes and acentrosomal poles began to splay (Figure 2C, Video 3). Splaying occurred at both poles (Figure 2C, arrowheads) and spindle length increased over time. To visualize the movement and alignment of chromosomes during this process, we repeated this experiment in our GFP::tubulin and GFP::histone Dynein AID strain (Figure 2D). As before, metaphase-arrested spindles were quickly disrupted upon dissection into auxin solution (Videos 4 and 5); poles began to splay (Figure 2D, arrowheads) and spindles again lengthened. Despite these changes, chromosomes and associated microtubule bundles were able to remain relatively aligned. To verify that the phenotypes were not artifacts of metaphase arrest, we repeated this experiment without emb-30(RNAi) and saw the same pole defects (Figure 2 - figure supplement 2, 
162

163

164

165

166

167

168

169

170

171

172

173

174

175

176

177

178

179

180

181

182

183

184

185

186

187

Videos 6 and 7). These embryos subsequently progressed into anaphase, albeit with defects in spindle positioning, as observed in fixed imaging. Altogether, these data demonstrate that dynein is not only required to focus poles during meiotic spindle assembly, but is also required to maintain the integrity of acentrosomal poles once they have been established.

\section{Dynein acts at spindle poles to promote pole formation and integrity}

Despite the pole defects observed following dynein depletion, spindle microtubules remained aligned along a single axis and bipolarity was not lost, likely because microtubule bundles in the central region remained intact to stabilize the spindle. To test this hypothesis, we sought to deplete dynein from a monopolar spindle, generated by depleting the kinesin-12 motor KLP-18; these structures have a single focused pole and therefore no area of microtubule overlap that could stabilize the structure if the pole is disrupted (Wignall and Villeneuve, 2009). Previously, we found that partial dynein inhibition on a monopolar spindle led to chromosomes moving a substantial distance away from the center of intact monopoles (Muscat et al., 2015); the AID approach now allows us to revisit these results with more complete dynein depletion.

First, we quantified embryos in intact dynein AID worms treated with klp-18(RNAi), and we found that long-term (4 hour) auxin-treated embryos rarely displayed a monopolar spindle (Figure 3A). Instead, chromosomes were dispersed throughout the embryo, retaining association to some microtubule bundles. IF imaging following acute auxin treatment confirmed this result; ASPM-1 labeling revealed that monopoles had begun breaking apart, ejecting chromosomes and associated microtubule bundles into the cytoplasm (Figure 3B). In some cases, the entirety of the monopole disappeared and all chromosomes retained their own laterally-associated microtubule bundles. We observed the same phenotype using live ex utero imaging; when embryos containing monopolar spindles were dissected into auxin solution, we observed rapid breakdown of the monopole on a timescale similar to that required for full dynein depletion (about three minutes) in previous experiments (Figure 4A, 4B, and Video 8). As the 
188

189

190

191

192

193

194

195

196

197

198

199

200

201

202

203

204

205

206

207

208

209

210

211

212

213

monopolar spindle began to fragment, microtubule bundles clearly remained associated with individual chromosomes via lateral associations (Figure 4A, arrowheads), as seen in fixed imaging (Figure 3B). These results demonstrate an essential role for dynein in maintaining pole integrity and suggest that in the case of dynein depletion from bipolar spindles, the intact overlap zone stabilizes the spindle even though poles are completely disrupted.

The fact that dynein localizes to poles and depletion has such a dramatic effect on pole integrity suggests that dynein might act at the pole itself to stabilize this structure. To test this hypothesis, we sought to delocalize dynein from poles and assess effects on pole integrity. The microtubule-associated protein NuMA has been shown to direct cytoplasmic dynein to microtubule minus ends and to promote pole focusing during mitosis and pole-directed transport of newly formed minus ends (Elting et al., 2014; Hueschen et al., 2017). Additionally, the $C$. elegans homolog of NuMA, LIN-5, has been shown to target dynein to spindle poles in oocytes to promote spindle rotation (van der Voet et al., 2009). Therefore, we depleted LIN-5 using RNAi to determine if delocalizing dynein from poles would have the same phenotypes as dynein depletion. LIN-5 depletion from bipolar spindles caused pole defects that were identical to 4hour dynein AID (Figure 3 - figure supplement 1A); labeling with ASPM-1 revealed splayed poles and dispersed minus ends throughout the spindle (Figure 3 - figure supplement 1B). Additionally, depletion of LIN-5 from monopolar spindles caused breakdown of monopoles (Figure 3 - figure supplement 1B). Dynein AID depletion combined with lin-5(RNAi) had similar pole defects as lin-5(RNAi) or Dynein depletion alone (Figure 3 - figure supplement 1B), supporting the view that dynein and LIN-5 operate in the same pathway (van der Voet et al., 2009).

Another protein that acts with LIN-5 to target dynein to poles and promote spindle rotation is ASPM-1. Additionally, depletion of ASPM-1 has been shown to result in longer oocyte spindles with splayed poles (van der Voet et al., 2009; Connolly et al., 2014; Laband et al., 2017) (Figure 3C), similar to the phenotype of dynein AID (Figure 1D-F, Figure 2), which 
suggests a role in pole focusing. Consistent with this, we found that ASPM-1 depletion caused the breakdown of monopolar spindles (Figure $\mathbf{3 C}$ ), again suggesting that proper localization of dynein to poles is required for pole integrity. Notably, monopole breakdown was not as severe following lin-5 or aspm-1 RNAi as that seen in the acute dynein AID depletions; for lin-5 and aspm-1 RNAi conditions, only $\sim 20 \%$ of monopoles completely broke down (15/73 and 14/61, respectively) and roughly half of embryos displayed partial breakdown (44/73 and 29/61, respectively). This might be caused by incomplete depletion due to the variable efficiency of RNAi, or perhaps because dynein is still present in these embryos and could be partially functional even though it is mislocalized. Regardless, our results suggest that dynein localization to spindle poles is required for the efficient focusing and stabilization of these 224 structures.

Individualized chromosomes can undergo an anaphase-like segregation in the absence

\section{of KLP-18 and dynein}

In addition to revealing the dynamics of monopole breakdown, our live imaging of 2015). In auxin-treated embryos, as filming continued past the full breakdown of the monopole, we were surprised to see that each individual chromosome began to segregate (Figure 4A, 4B, Video 8, 10). This segregation was incredibly striking, as it occurred synchronously across the embryo. The fact that we observed these anaphase-like movements in the absence of both

237 KLP-18 and dynein suggests that neither motor is absolutely required for chromosome 238 segregation in oocytes. 
When we compared the segregation distance of chromosomes from these videos to control anaphases (bipolar spindles with no auxin), we found that chromosomes segregated to a similar final distance on the dynein-depleted $k l p-18(R N A i)$ mini-anaphase spindles (Figure 4 -

figure supplement 1). However, mini anaphases did not appear to segregate as far as dyneindepleted bipolar anaphases, where KLP-18 was not depleted. This observation raises the possibility that, even though chromosomes can segregate without KLP-18, this motor may normally contribute to anaphase spindle elongation through microtubule sliding. This contribution has been difficult to test due to the monopolar spindle phenotype that is quickly generated upon removal of KLP-18 (Wolff et al., 2021), which prevents normal anaphase segregation from occurring.

To further characterize these segregations, we performed fixed imaging. This analysis revealed that the mini-anaphases had key hallmarks of normal anaphase spindles; when chromosomes were still close together we observed lateral microtubule bundles running alongside the separating chromosomes (Figure 4C, "early anaphase"), and as chromosomes segregated further and spindle length increased, microtubules were largely localized between chromosomes (Figure 4C, "late" anaphase). This suggests that, like normal anaphase spindles, these mini spindles undergo morphological changes and anaphase-B-like spindle elongation as they drive chromosomes apart.

The fact that we observed chromosome segregation with hallmarks of normal anaphase led us to hypothesize that some local microtubule reorganization must be occurring after monopole breakdown; without a bipolar distribution of microtubule minus ends, it should not be possible for chromosomes to segregate away from each other in the anaphase-like manner we observed. In live imaging, we were able to visualize a brief window between full monopole 
breakdown and the initiation of segregation (Figure 4A, 4B, Video 8, 10). During this window, microtubule bundles shortened around chromosomes, and microtubule ends could be clearly identified on both sides of individual chromosomes prior to the initial segregation (Figure 4B, arrowheads). Subsequently, an anaphase-like spindle could be observed until the completion of segregation. In order to better characterize this possible reorganization, we returned to IF imaging. In a few occasions we were able to capture this transitional period; ASPM-1 labeling could be clearly seen on both sides of these mini spindles, with some enrichment towards areas resembling spindle poles (Figure 5A).

This imaging suggested that microtubules might reorganize into a mini-bipolar spindle that could facilitate chromosome segregation. To test this idea, we assessed the localization of a well-studied spindle protein, the PRC1 homolog SPD-1. SPD-1 localizes to the anaphase spindle midzone in oocytes, marking a region of antiparallel microtubule overlap (Hattersley et al., 2016; Gigant et al., 2017; Mullen and Wignall, 2017), and we found that SPD-1 was also at this location in the absence of dynein (Figure 5B, row 4). In intact and disassembling monopoles, SPD-1 is not localized to microtubules, as it only associates with the spindle in anaphase. However, once chromosomes had been ejected into the cytoplasm and microtubules reorganized around chromosomes, SPD-1 staining could be clearly observed on mini anaphase spindles (Figure 5B, bottom row), demonstrating that they have a region of anti-parallel overlap in the center.

In addition to SPD-1, we also assessed the localization of the ring complex (RC), a collection of proteins that forms a ring around the center of each chromosome in oocytes. RCs aid in chromosome congression (Wignall and Villeneuve, 2009; Muscat et al., 2015; Pelisch et al., 2017; Hollis et al., 2020), and then are released from chromosomes during anaphase; they remain as a ring between segregating chromosomes and then are disassembled as anaphase progresses (Dumont et al., 2010; Muscat et al., 2015; Davis-Roca et al., 2017; Pelisch et al., 
a post-translational modification found in the RC (Pelisch et al., 2017). In control and dynein depletion conditions, SUMO-marked RCs were removed from chromosomes during anaphase and were localized between segregating chromosomes, as expected (Figure $\mathbf{5}$ - figure supplement 1). When introducing monopolar breakdown, SUMO could be seen on the RC as individual chromosomes detached from the dissolving monopole. In mini anaphases, SUMO labeling persisted on what appeared to be a singular disassembling RC between each segregating chromosome pair. Taken together, these findings support the idea that microtubules reorganize into miniature spindles that recapitulate key aspects of normal anaphase.

The kinesin-5 family motor BMK-1 provides an outward sorting force that allows spindle reorganization in the absence of KLP-18 and dynein either oocytes or embryos (Bishop et al., 2005) and only minor effects on chromosome segregation rates (Saunders et al., 2007; Laband et al., 2017). We therefore hypothesized that BMK-1 could be providing a supplementary outward sorting force that is normally masked by the contributions of KLP-18. 
To probe this hypothesis, we first sought to confirm the localization of BMK-1 on meiotic spindles and to test if this motor was localized to microtubules under the monopole breakdown conditions. BMK-1 was broadly associated with bipolar metaphase and anaphase spindles, in both control and dynein-depletion conditions (Figure 6A). In intact monopolar spindles, BMK-1 also localized to microtubules. Importantly, when we depleted dynein and induced monopole breakdown, BMK-1 was still localized to microtubules (Figure 6A, zooms), placing it in a location where it could contribute to microtubule reorganization.

To test if BMK-1 was necessary for the formation of miniature anaphases, we utilized two bmk-1 mutants: 1) a previously-characterized allele, bmk-1(ok391), that introduces a premature stop codon in the motor domain (Bishop et al., 2005), and 2) a new deletion of the entire bmk-1 locus generated using CRISPR-Cas9 (bmk-1(syb3914)). To validate these deletions, we utilized IF imaging with an a-BMK-1 antibody and confirmed that BMK-1 was no longer present on the meiotic spindle in either mutant (Figure 6 - figure supplement 1A, 1B). We then generated monopolar spindles, performed acute dynein AID, and performed IF in the bmk-1(ok391) background; in this bmk-1 mutant we were unable to observe any mini anaphase spindles, even though monopole breakdown still occurred (Figure 6B, 6C); this suggested that removal of BMK-1 function abolished microtubule reorganization and prevented chromosome segregation. To confirm this result, we crossed the bmk-1 CRISPR deletion mutant into our Dynein AID live imaging strain (expressing GFP::tubulin and GFP.::histone to visualize the spindle) and performed ex utero imaging to determine if mini anaphases could still form (Figure 6D). In klp-18(RNAi), bmk-1(syb3914) worms in the absence of auxin, monopolar spindles formed and then chromosomes moved back towards the pole in a manner indistinguishable from normal monopolar anaphase (Video 11). When auxin was added to deplete dynein, monopolar spindles broke down as expected and individual chromosomes remained associated 341 with microtubule bundles. However, as time elapsed, there were no signs of segregation, 342 microtubule density decreased, and chromosomes remained in the cytoplasm with no 
343 discernable anaphase spindle forming (Video 12). These data support the hypothesis that BMK-

3441 provides outward sorting force on microtubules during oocyte meiosis, redundant to the forces

345 produced by KLP-18. 


\section{DISCUSSION}

\section{Dynein is essential for acentrosomal pole focusing throughout meiosis}

Collectively, these data have contributed to a more complete model for how acentrosomal spindles are assembled and stabilized during oocyte meiosis (Figure 7). Dynein activity is required throughout the meiotic divisions to establish and maintain focused poles, and its removal via acute AID generated spindle defects within minutes. Dynein depletion increased the length of bipolar oocyte spindles and led to increased dispersion of microtubule minus ends across the spindle. When performing these depletions on a monopolar spindle, the monopole completely broke apart and microtubule bundles were ejected into the cytoplasm, demonstrating that dynein is required to stitch minus ends together into a pole structure. Remarkably, we also found that after the monopole disassembled, microtubules were able to reorganize into a "miniature" spindle capable of facilitating an anaphase-like chromosome segregation. This provides additional evidence that dynein is not essential for chromosome segregation, as has been suggested by previous studies that used partial dynein depletion and/or temperaturesensitive mutants (Muscat et al., 2015; McNally et al., 2016; Laband et al., 2017). In our dynein AID depletions, unarrested bipolar spindles progress through anaphase and mini spindles also undergo anaphase-like segregation, reaching comparable segregation distances to that of normal anaphase spindles. This corroborates a recent study that also used auxin-inducible degradation to assess chromosome segregation (Danlasky et al., 2020); those dynein depletion experiments demonstrate the same segregation distance trends shown here. Moreover, our studies also show that anaphase-B-like spindle elongation can occur in the absence of KLP-18. Although it is possible that KLP-18 may contribute to outward sliding of microtubules during wild type anaphase, our work shows that this motor is not absolutely required for anaphase spindle elongation and demonstrates that there must be other factors that can perform this function. 


\section{Multiple motors cooperate in C. elegans oocyte meiosis to effectively form and stabilize}

\section{an acentrosomal bipolar spindle}

Our work also accentuates the importance of balanced forces within a bipolar meiotic spindle; we found that loss of dynein activity had strong phenotypes that manifested rapidly, within two minutes of auxin treatment. Notably, acute dynein depletion caused spindle lengthening, suggesting that dynein may normally provide an inward force on the spindle, as has been demonstrated in previous studies of mitosis (Ferenz et al., 2009; van Heesbeen et al., 2014). Recently, our lab also demonstrated that inactivation of KLP-18 in stable, bipolar spindles caused spindle shortening followed by collapse of microtubule minus ends into a monopolar spindle (Wolff et al., 2021). This collapse is due to a loss of the outward force provided by KLP-18, theoretically enabling the inward force provided by dynein to dominate and highlighting how quickly an imbalance of these motor forces can lead to gross defects in spindle bipolarity. In the current study, removing dynein from oocytes that already lacked KLP-18 resulted in a catastrophic breakdown of the spindle, releasing individual chromosomes into the cytoplasm. Going forward, it would be interesting to acutely remove these proteins at the same time to see if oocyte spindles are able to maintain bipolarity and directly test whether these motors antagonize each other in $C$. elegans.

While KLP-18/kinesin-12 provides the major outward force in C. elegans oocyte meiosis, this critical function is performed by kinesin-5 in mouse oocytes (Schuh and Ellenberg, 2007) and in mitosis in many organisms (reviewed in (Mann and Wadsworth, 2019)). Previous studies have shown that dynein and kinesin-5 antagonize each other in these organisms; inhibition of both motors simultaneously enables bipolar spindle formation (Mitchison et al., 2005;

Tanenbaum et al., 2008; Ferenz et al., 2009; van Heesbeen et al., 2014). Interestingly, it has been shown that Kif15/kinesin-12 is capable of providing a supplemental outward force that can support bipolarity when kinesin-5 is inhibited (Tanenbaum et al., 2009; Vanneste et al., 2009; Raaijmakers et al., 2012; Sturgill and Ohi, 2013; Sturgill et al., 2016). Here, we have provided 
evidence that these roles have been reversed in $C$. elegans oocyte meiosis; BMK-1/kinesin-5 appears to be providing a redundant outward sorting force that was only detectable once we had removed KLP-18 and dynein from the meiotic spindle. In the future, it would be fascinating to perform KLP-18/DHC-1 double depletions in a strain lacking BMK-1 function to further probe the relationship between these three motors during acentrosomal spindle formation in $C$. elegans.

While this work has expanded our understanding of the role BMK-1 plays in C. elegans oocyte meiosis, further experimentation will be valuable for understanding the exact mechanism of BMK-1 function in the context of a normal, bipolar meiotic spindle. Biophysical assays to determine motor walking speed and force generation would help frame how much BMK-1 contributes in comparison to other meiotic motors such as KLP-18. Also, since kinesin-5 activity is known to be regulated in other systems by kinases and protein-protein interactions (reviewed in (Mann and Wadsworth, 2019)), it would be beneficial to determine if BMK-1 has any interacting partners that provide some regulation of function. Aurora B kinase (AIR-2) has been shown to be required for BMK-1 spindle localization and AIR-2 can phosphorylate BMK-1 in vitro (Bishop et al., 2005) implicating AIR-2 in BMK-1 regulation. Inhibition of AIR-2's kinase activity also leads to collapsed oocyte spindles (Divekar et al., 2021a), consistent with a role for AIR-2 in regulating spindle force generation. However, it is likely that other factors also regulate BMK-1. Finally, a broader understanding of why the relationship of kinesin- 5 and kinesin-12 in oocyte meiosis has been seemingly switched between different organisms could provide some valuable context to the optimization of acentrosomal spindles over evolutionary time.

\section{Different acentrosomal pole proteins have distinct roles in pole coalescence and stability}

Our analysis of ASPM-1 and LIN-5 provides further evidence that these proteins are directly influencing acentrosomal pole organization and stability by directing cytoplasmic dynein localization. In either aspm-1 or lin-5 RNAi, spindle phenotypes were nearly identical to those 
423

424

425

426

427

428

429

430

431

432

433

434

435

436

437

438

439

440

441

442

443

444

445

446

447

448

seen in Dynein AID experimentation. Additionally, when introducing double depletions with $k / p$ 18(RNAi), partial breakdown of monopolar spindles could be observed. Neither double depletion condition was as extreme as Dynein AID phenotypes, but this could be attributed to either inconsistent RNAi efficiency (more common with double depletions) or to partial dynein activity despite it being unable to properly localize to poles. The shared phenotype across depletions of these proteins suggests that these pole proteins function in a single pathway; this provides some credence to the concept that acentrosomal poles could contain a cross-linked network of interacting pole proteins that provide cohesion and stability. However, recent unpublished work from our lab has made it clear that not all pole protein depletions yield the same defects in spindle bipolarity. Acute depletion of ZYG-9, a homolog of the microtubule polymerase XMAP215, disrupts spindle integrity in a manner that does not resemble dynein AID depletions. While dynein depletion splays spindle poles, microtubule bundles appear to remain stable and unperturbed, generating a rectangular spindle that can still progress through meiosis (albeit with spindle rotation defects). However, acute ZYG-9 depletion leads to a disorganized multipolar spindle that is highly prone to segregation errors, suggesting a broader role of ZYG-9 not just restricted to poles.

From these recent studies, it is clear that multiple groups of proteins are required to establish and maintain acentrosomal poles. However, despite having similar or nearly identical localizations in the meiotic spindle, pole proteins have separable functions that impact pole stability in unique ways. Rather than all pole proteins interacting as a cohesive, cross-linked network around microtubule minus ends, it seems more plausible that different groups of proteins work in parallel to form and stabilize acentrosomal poles. These degron-based depletions with essential pole proteins have allowed for further mechanistic understanding of how each pole protein is contributing to spindle stability. There remain numerous pole proteins that could be subjected to rapid depletion via the AID system; the ability to probe the roles of essential proteins in both spindle assembly and maintenance is vital to building a more robust 
bioRxiv preprint doi: https://doi org/10.1101/2021.09.09.459640; this version posted September 9, 2021. The copyright holder for this preprint (which was not certified by peer review) is the author/funder, who has granted bioRxiv a license to display the preprint in perpetuity. It is made available under aCC-BY 4.0 International license.

449 model of how meiotic spindles are able to achieve bipolarity in the absence of centrosomes to 450 faithfully congress and segregate chromosomes. 
MATERIALS AND METHODS

452

C. elegans strains used

\begin{tabular}{|c|c|c|}
\hline Name & Description & Genotype \\
\hline CA1215 & $\begin{array}{l}\text { dhc-1::degron::GFP } \\
P_{\text {sun- } 1:: T I R 1:: m R u b y}\end{array}$ & dhc-1(ie28[dhc-1::degron::GFP]) I; ieSi38 IV \\
\hline PHX3914 & $\begin{array}{l}\text { dhc-1::degron::GFP } \\
P_{\text {sun-1::TIR } 1:: m R u b y} \\
\text { GFP::H2B } \\
\text { GFP::tubulin } \\
\Delta b m k-1\end{array}$ & $\begin{array}{l}\text { dhc-1(ie28[dhc-1::degron::GFP]) I; unc- } \\
\text { 119(ed3) III; ruls32[unc-119(+) pie- } \\
\text { 1::gfp::h2b] III; ruls57[unc-119(+) pie- } \\
\text { 1::GFP::tubulin]; ieSi38 IV; bmk-1(syb3914) } \\
\text { V }\end{array}$ \\
\hline SMW22 & $\begin{array}{l}P_{\text {sun- } 1:: T I R 1:: m R u b y} \\
\text { mCherry::tubulin }\end{array}$ & $\begin{array}{l}\text { unc-119(ed3) III; wels21 [pJA138 (pie- } \\
1:: m \text { Cherry::tubulin::pie-1)]; ieSi38 IV }\end{array}$ \\
\hline SMW31 & $\begin{array}{l}P_{\text {sun- } 1:: T I R 1:: m R u b y} \\
\text { GFP::H2B } \\
\text { GFP::tubulin }\end{array}$ & $\begin{array}{l}\text { unc-119(ed3) III; ruls32[unc-119(+) pie- } \\
\text { 1::gfp::h2b] III; ruls57[unc-119(+) pie- } \\
\text { 1::GFP::tubulin]; ieSi38 IV }\end{array}$ \\
\hline SMW46 & $\begin{array}{l}\text { dhc-1::degron::GFP } \\
P_{\text {sun- } 1:: T I R 1:: m R u b y} \\
\text { GFP::H2B } \\
\text { GFP::tubulin }\end{array}$ & $\begin{array}{l}\text { dhc-1(ie28[dhc-1::degron::GFP]) I; unc- } \\
\text { 119(ed3) III; ruls32[unc-119(+) pie- } \\
\text { 1::gfp::h2b] III; ruls57[unc-119(+) pie- } \\
1:: G F P:: t u b u l i n] ; ~ i e S i 38 ~ I V\end{array}$ \\
\hline SMW47 & 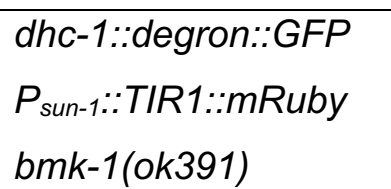 & $\begin{array}{l}\text { dhc-1(ie28[dhc-1::degron::GFP]) I; ieSi38 IV; } \\
\text { bmk-1(ok391) V }\end{array}$ \\
\hline SMW48 & $\begin{array}{l}\text { dhc-1::degron::GFP } \\
P_{\text {sun-1::TIR1::mRuby }} \\
\text { mCherry::tubulin }\end{array}$ & $\begin{array}{l}\text { dhc-1(ie28[dhc-1::degron::GFP]) I; wels21 } \\
\text { [pJA138 (pie-1::mCherry::tub::pie-1)]; ieSi38 } \\
\text { IV }\end{array}$ \\
\hline SV1005 & $b m k-1(o k 391)$ & bmk-1(ok391) V \\
\hline
\end{tabular}




\section{Generation of C. elegans strains}

CA1215 was generated via CRISPR/Cas9 editing of the endogenous $d h c-1$ locus (Zhang et al., 2015).

PHX3914 was generated via CRISPR/Cas9 editing of the endogenous bmk-1 locus by

SunyBiotech.

SV1005 was a knockout strain generated by the C. elegans Deletion Mutant Consortium

SMW46-SMW48 were generated via crossing of males and hermaphrodites of two strains and screening multiple generations of progeny to ensure homozygosity of all desired traits.

SMW46: Crossed males of SMW31 with CA1215 hermaphrodites

SMW47: Crossed males of CA1215 with SV1005 hermaphrodites

SMW48: Crossed males of CA1215 with SMW22 hermaphrodites

\section{RNAi Feeding}

From a RNAi library (Kamath et al., 2003), individual RNAi clones were picked and for 5-6 days. In experiments utilizing lin-5(RNAi), L1 worms were plated on EV (empty vector) control RNAi plates for 3 days, then transferred to lin-5(RNAi) plates 72 hours prior to fixation.

\section{Immunofluorescence and antibodies}

Adult worms, grown on either EV (empty vector) control RNAi or experimental RNAi, 
Lysine-coated glass slide. Worms were dissected, then fixed via freeze cracking and plunging into $-20^{\circ} \mathrm{C} \mathrm{MeOH}$ as described in (Oegema et al., 2001). Embryos were fixed for 40-45 minutes, rehydrated in PBS, and blocked in AbDil (PBS with 4\% BSA, 0.1\% Triton-X-100, 0.02\% NaN $\mathrm{N}_{3}$ ) overnight at $4^{\circ} \mathrm{C}$. Primary antibodies were diluted in AbDil and incubated with sample overnight at $4^{\circ} \mathrm{C}$. The following day, samples were moved to room temperature and rinsed three times in PBST (PBS with $0.1 \%$ Triton-X-100), then incubated with secondary antibodies (diluted in PBST) for 2 hours. Next, samples were washed three times in PBST again, and incubated with mouse anti- $\alpha$-Tubulin-FITC (diluted in PBST) for 2 hours. Again, samples were washed three times in PBST, then incubated with Hoëscht (1:1000 in PBST) for 15 minutes. Finally, samples were washed two times in PBST, mounted in 0.5\% p-phenylenediamine, $20 \mathrm{mM}$ Tris- $\mathrm{Cl}, \mathrm{pH} 8.8$, $90 \%$ glycerol, then sealed with nail polish and stored at $4^{\circ} \mathrm{C}$.

Primary antibodies used in this study: rabbit-a-ASPM-1 (1:5000, gift from Arshad Desai), rabbit- $\alpha-B M K-1$ (1:250, gift from Jill Schumacher), mouse- $\alpha-T u b u l i n-F I T C ~(1: 500$, DM1 $\alpha$, Sigma), mouse- $\alpha-G F P$ (1:250, 3E6, Invitrogen), mouse- $\alpha-S U M O$ (1:500, gift from Federico Pelisch), and rabbit-a-SPD-1 (Mullen and Wignall, 2017). All rabbit and mouse Alexa-fluor secondary antibodies (Invitrogen) were used at 1:500.

\section{Ex utero live imaging}

Fifteen adult worms, grown on either control RNAi or experimental RNAi, were picked

499 into a $10 \mu \mathrm{L}$ drop of Meiosis Media in the center of a custom-made apparatus for live imaging 500 (Laband et al., 2018; Divekar et al., 2021b). All worms were quickly dissected, and an eyelash 501 pick was used to push remaining worm bodies to the outside of the drop, leaving only the 502 embryos in the center to avoid disruption from worm movement during the imaging process.

503 Vaseline was laid in a ring around the drop through a syringe, and a $18 \times 18 \mathrm{~mm} \# 1$ coverslip was 504 laid on top of the Vaseline ring, sealing the drop. This sealed slide was moved immediately to 
505

506

507

508

509

510

511

512

513

514

515

516

517

518

519

520

521

522

523

524

525

526

527

528

529

530

the Spinning Disk stage and inverted, allowing embryos to float down to the surface of the coverslip and be subsequently imaged.

\section{Microscopy}

All fixed imaging was performed on a DeltaVision Core deconvolution microscope with a 100x objective (NA = 1.4) (Applied Precision). This microscope is housed in the Northwestern University Biological Imaging Facility supported by the NU Office for Research. Image stacks were obtained at $0.2 \mu \mathrm{m}$ z-steps and deconvolved using SoftWoRx (Applied Precision). All immunofluorescence images in this study were deconvolved and displayed as full maximum intensity projections of data stacks encompassing the entire spindle structure (typically $\sim 4-6 \mu \mathrm{m}$ ).

All live imaging was performed using a spinning disk confocal microscope with a $63 \mathrm{x} \mathrm{HC}$ PL APO 1.40 NA objective lens. A spinning disk confocal unit (CSU-X1; Yokogawa Electric Corporation) attached to an inverted microscope (Leica DMI6000 SD) and a Spectral Applied Imaging laser merge ILE3030 and a back-thinned electron-multiplying charge-coupled device (EMCCD) camera (Photometrics Evolve 521 Delta) were used for image acquisition. The microscope and attached devices were controlled using Metamorph Image Series Environment software (Molecular Devices). Typically, ten to fifteen z-stacks at $1 \mu \mathrm{m}$ increments were taken every 15-30 seconds at room temperature. Images were processed using ImageJ; images are shown as maximum intensity projections of the entire spindle structure. The spinning disk microscope is housed in the Northwestern University Biological Imaging Facility supported by the NU Office for Research.

\section{Acute auxin treatment of $C$. elegans}

Acute auxin treatments utilized in immunofluorescence experiments were performed as in (Divekar et al., 2021a). Briefly, a Meiosis Media solution containing $1 \mathrm{mM}$ auxin was prepared using a $200 \mathrm{mM}$ stock of auxin dissolved in $100 \% \mathrm{EtOH}$ and kept on ice. Worms were picked 
into $10 \mu \mathrm{L}$ drops of Media+auxin, and then the slides were placed inside a custom-made humidity chamber to avoid evaporation of the drop. Following incubation for desired time (30-45 minutes), worms were immediately dissected and subjected to standard IF protocol described above.

For acute auxin treatments utilized in ex utero live imaging, a Meiosis Media solution containing $100 \mu \mathrm{M}$ auxin was made from a $200 \mathrm{mM}$ stock of auxin dissolved in $100 \% \mathrm{EtOH}$. Worms were picked into $10 \mu \mathrm{L}$ drops of Media+auxin, and dissected on a custom-made live imaging slide apparatus described above. Slides were moved quickly to the Spinning Disk, and acquisition was started as soon as an embryo could be found in order to begin imaging prior to full protein depletion. More detailed auxin treatment protocols are described in (Divekar et al., 2021b).

\section{Ethanol Fixation for Germline Counting}

SMW46 worms were subjected to EV Control RNAi, utilizing methods described above, and were grown to adulthood over 5 days. Four hours prior to fixation, worms were either transferred to EV Control RNAi (with $1 \mathrm{mM}$ auxin) or left on their original RNAi plates. For each biological replicate, $\sim 40$ adults were picked off their respective plates into a $10 \mu \mathrm{L}$ drop of M9 on a standard glass slide, and a small piece of Whatman paper was used to absorb excess M9, with care being used to avoid pulling worms onto filter paper. Once worms had formed a tight cluster with little residual $\mathrm{M} 9$, a $10 \mu \mathrm{L}$ drop of $100 \% \mathrm{EtOH}$ was quickly pipetted onto the worms. Within seconds, worms became rigid and straight; once the first EtOH drop had dried, another $10 \mu \mathrm{L}$ drop was applied and allowed to completely dry. After the third drop was added and dried, a $10 \mu \mathrm{L}$ drop of $50 \%$ Vectashield Mounting Media (Vector Laboratories $\mathrm{H}-1000$ ) and 50\% M9 was placed onto the worms, and a $18 \times 18 \mathrm{~mm}$ coverslip was gently placed on top. Excess media was aspirated away, slides were sealed with nail polish, and stored at $4^{\circ} \mathrm{C}$ (typically imaged within a week of fixation). 


\section{Data analysis}

558 Figure 1D: Quantifications were made by viewing whole Dynein AID worms expressing GFP::tubulin and GFP::histone and observing three positions in each worm (-1 Oocyte, Spermatheca, +1 Embryo) in both gonad arms. When spindle morphology was clear enough to confidently categorize, embryos (already sorted based on location) were then counted into one of four categories (Microtubule Cage, Unfocused, Focused, Anaphase). Number of embryos counted across all slides $(n)$ are placed above each respective location and condition. Each bar comprises embryo counts from at least 4 biological replicates.

565 Figure 1E and 1F: Categorization of acentrosomal poles into either focused or unfocused/splayed was done by eye, looking at both microtubule and ASPM-1 channels. In any case where microtubule bundles were clearly separated from one another at an acentrosomal pole, with multiple ASPM-1 foci, that spindle was considered unfocused/splayed. Quantifications of acentrosomal pole splaying were made by categorizing multiple fixed images of embryos; numbers of embryos counted across all slides (n) are placed above each respective condition.

571 Each bar comprises embryos imaged across 3 biological replicates.

572 Figure 2A and 2B: Categorization of acentrosomal poles into either focused or

573 unfocused/splayed was done by eye, looking at both microtubule and ASPM-1 channels. In any case where microtubule bundles were clearly separated from one another at an acentrosomal pole, with multiple ASPM-1 foci, that spindle was considered unfocused/splayed. Quantifications of acentrosomal pole splaying were made by categorizing multiple fixed images of embryos; numbers of embryos counted across all slides ( $n$ ) are placed above each respective condition.

578 Each bar comprises embryos imaged across at least 3 biological replicates.

579 Figure 2 - figure supplement 1A and 1B: Utilizing FIJI, a rectangular ROI (12 $\mu \mathrm{m}$ or $14 \mu \mathrm{m}$ 580 wide by $4 \mu \mathrm{m}$ tall) was used on all images of a single condition to measure the average pixel 581 intensity of ASPM-1 across the whole width of the ROI via the Plot Profile Tool. These values 582 were then averaged from all images in that condition to produce a single value for ASPM-1 
intensity at each pixel across the standardized $12 \mu \mathrm{m} / 14 \mu \mathrm{m}$ length. This was plotted as a solid line for each condition, with shaded areas above and below the line representing the SEM. All images were taken with the same exposures for all channels. Spindles were sum projected over 20 slices $(0.2 \mu \mathrm{m}$ step size $)$ for each image prior to measurement. Each condition was averaged using images from at least 3 biological replicates. Spindle length measurements were done in Imaris. Poles were projected into 3D volumes using the Surfaces Tool (based off of fluorescence intensity), and the center of each volume was determined. The exact micron distance between the designated center of the two volumes was measured, and this distance was averaged across images of the same condition. Quantifications were arranged as boxplots, and statistical significance was determined via a two-tailed t test. Each condition was averaged using images from at least 3 biological replicates.

Figure 3A: Quantifications were made by viewing whole Dynein AID worms expressing GFP::tubulin and GFP::histone and observing all +1 embryos. When spindle morphology was clear enough to confidently categorize, embryos were then counted as either monopolar (a single structure with chromosomes fanned away from center) or undergoing breakdown (noticeable separation of microtubules and chromosomes). Number of embryos counted across all slides $(n)$ are placed above each respective location and condition. Each bar comprises embryo counts from 3 biological replicates.

601 Figure 3 - figure supplement 1A: Quantifications were made by viewing whole Dynein AID worms expressing GFP::tubulin and GFP::histone and observing two positions in each worm (Spermatheca and +1 Embryo) in both gonad arms. When spindle morphology was clear enough to confidently categorize, embryos (already sorted based on location) were then counted into one of three categories (Unfocused, Focused, Anaphase). Number of embryos counted across all slides $(n)$ are placed above each respective location and condition. Each bar 607 comprises embryo counts from at least 3 biological replicates. 
608 Figure 4 - figure supplement 1: Timepoints from live imaging videos were analyzed utilizing

609 Imaris. Chromosomes were rendered into 3D surfaces using the "Surfaces" tool, and the center

610 of each volume was determined. For each chromosome that was within the z-stack throughout

611 the entirety of the timelapse, measurements of the distance between the center of segregating

612 chromosomes were taken. These distances were averaged at each 15 second timepoint, and

613 each timelapse was standardized to the same starting point (onset of Anaphase A) to allow for

614 further averaging between separate videos. The average segregation distance across each

615 condition was plotted (number of videos in each condition represented by $n$ ), and shaded area

616 represents SEM of each average.

617 Figure 6B and 6C: Classification of monopolar spindles into one of three categories

618 (Breakdown, Mini Anaphases, No Segregation) was done by eye, looking at DNA, microtubule,

619 and ASPM-1 channels. In any case where some number of bivalents were clearly separated

620 from the monopolar spindle, yet a distinct ASPM-1 monopole remained with some number of

621 attached bivalents, was classified as "Breakdown". Whenever all six bivalents could be seen

622 dispersed in the cytoplasm with no remaining ASPM-1 monopole, and appeared to have some

623 segregation between chromosomes, that embryo was considered "Mini Anaphases". Any

624 oocytes containing dispersed chromosomes that were observed without any indication of

625 segregation or an anaphase spindle were classified as "No Segregation". Numbers of embryos

626 counted across all slides ( $\mathrm{n}$ ) are placed above each respective condition. Each bar comprises

627 embryos imaged across 5 biological replicates. 
Table 1 (corresponds to Figure 1D)

\begin{tabular}{|c|c|c|c|c|c|}
\hline Location & Treatment & MT Cage & Unfocused & Focused & Anaphase \\
\hline \multirow[t]{2}{*}{-1 oocyte } & No Auxin & $60 / 70(85.7 \%)$ & $10 / 70(14.3 \%)$ & N/A & $\mathrm{N} / \mathrm{A}$ \\
\hline & With Auxin & $47 / 54(87.0 \%)$ & $7 / 54$ (13.0\%) & N/A & N/A \\
\hline \multirow[t]{2}{*}{ Spermatheca } & No Auxin & N/A & $54 / 60(90.0 \%)$ & $6 / 60(10.0 \%)$ & N/A \\
\hline & With Auxin & N/A & $63 / 66(95.5 \%)$ & $3 / 66(4.5 \%)$ & N/A \\
\hline \multirow[t]{2}{*}{ +1 embryo } & No Auxin & N/A & $\begin{array}{l}8 / 160 \\
(5.0 \%)\end{array}$ & $\begin{array}{l}97 / 160 \\
(60.6 \%)\end{array}$ & $\begin{array}{l}55 / 160 \\
(34.4 \%)\end{array}$ \\
\hline & With Auxin & $\mathrm{N} / \mathrm{A}$ & $\begin{array}{l}84 / 121 \\
(69.4 \%)\end{array}$ & $\begin{array}{l}8 / 121 \\
(6.6 \%)\end{array}$ & $\begin{array}{l}29 / 181 \\
(24.0 \%)\end{array}$ \\
\hline
\end{tabular}

Table 2 (corresponds to Figure 1F)

\begin{tabular}{|c|c|c|c|}
\hline Treatment & Focused Poles & Unfocused Poles & Anaphases \\
\hline No Auxin & $35 / 60(58.3 \%)$ & $3 / 60(5.0 \%)$ & $22 / 60(36.7 \%)$ \\
\hline With Auxin & $3 / 54(5.6 \%)$ & $39 / 54(72.2 \%)$ & $12 / 54(22.2 \%)$ \\
\hline
\end{tabular}

Table 3 (corresponds to Figure 2B)

\begin{tabular}{|c|c|c|c|}
\hline Condition & Treatment & Focused Poles & Unfocused Poles \\
\hline \multirow{2}{*}{$\begin{array}{c}\text { Unarrested } \\
\text { control (RNAi)) }\end{array}$} & No Auxin & $102 / 114(89.5 \%)$ & $12 / 114(10.5 \%)$ \\
\cline { 2 - 4 } & With Auxin & $10 / 139(7.2 \%)$ & $129 / 139(92.8 \%)$ \\
\hline Arrested & No Auxin & $63 / 68(92.6 \%)$ & $5 / 68(7.4 \%)$ \\
\cline { 2 - 4 }$(e m b-30(R N A i))$ & With Auxin & $7 / 75(9.3 \%)$ & $68 / 75(90.7 \%)$ \\
\hline
\end{tabular}


Table 4 (corresponds to Figure 3A)

\begin{tabular}{|c|c|c|c|}
\hline Condition & Treatment & Monopolar & Breakdown \\
\hline \multirow{2}{*}{$k / p-18(R N A i)$} & No Auxin & $119 / 119(100.0 \%)$ & $0 / 119(0.0 \%)$ \\
\cline { 2 - 4 } & With Auxin & $9 / 125(7.2 \%)$ & $116 / 125(92.8 \%)$ \\
\hline
\end{tabular}

$\underline{\text { Table } 5 \text { (corresponds to Figure 3C) }}$

\begin{tabular}{|c|c|c|c|}
\hline Condition & $\underline{\text { Intact Monopole }}$ & Partial Breakdown & Full Breakdown \\
\hline aspm-1 (RNAi); & $18 / 61(29.5 \%)$ & $29 / 61(47.5 \%)$ & $14 / 61(23.0 \%)$ \\
$k l p-18(R N A i)$ & & & \\
\hline
\end{tabular}

Table 6 (corresponds to Figure 3 - figure supplement $1 \mathrm{~A}$ )

\begin{tabular}{|c|c|c|c|c|}
\hline Location & Condition & Focused Poles & Unfocused Poles & Anaphases \\
\hline \multirow{2}{*}{ Spermatheca } & control (RNAi) & $5 / 71(7.0 \%)$ & $66 / 71(93.0 \%)$ & N/A \\
\cline { 2 - 5 } & lin-5 (RNAi) & $4 / 83(4.8 \%)$ & $79 / 83(95.2 \%)$ & N/A \\
\hline +1 embryo & control (RNAi) & $72 / 115(62.6 \%)$ & $9 / 115(7.8 \%)$ & $34 / 115(29.6 \%)$ \\
\cline { 2 - 5 } & lin-5 (RNAi) & $10 / 188(5.3 \%)$ & $120 / 188(63.8 \%)$ & $58 / 188(30.9 \%)$ \\
\hline
\end{tabular}

Table 7 (corresponds to Figure 3 - figure supplement 1B)

\begin{tabular}{|c|c|c|c|}
\hline Condition & $\underline{\text { Intact Monopole }}$ & Partial Breakdown & Full Breakdown \\
\hline lin-5 (RNAi); & $14 / 73(19.2 \%)$ & $44 / 73(60.3 \%)$ & $15 / 73(20.5 \%)$ \\
klp-18 (RNAi) & & & \\
\hline
\end{tabular}


$\underline{\text { Table } 8 \text { (corresponds to Figure } 6 \mathrm{C} \text { ) }}$

\begin{tabular}{|c|c|c|c|c|}
\hline Condition & Treatment & Breakdown & Mini Anaphases & No Segregation \\
\hline \multirow{2}{*}{$k l p-18(R N A i)$} & $b m k-1(W T)$ & $82 / 130(63.1 \%)$ & $43 / 130(33.1 \%)$ & $5 / 130(3.8 \%)$ \\
\cline { 2 - 5 } & $b m k-1($ ok391) & $111 / 164(67.7 \%)$ & $0 / 164(0.0 \%)$ & $53 / 164(32.3 \%)$ \\
\hline
\end{tabular}

\section{ACKNOWLEDGMENTS}

629 We thank all members of the Wignall lab for support and discussion, and Emily Czajkowski,

630 Nikita Divekar, Hannah Horton, Juhi Narula, and lan Wolff for critical reading of the manuscript.

631 Additionally, we specifically thank graduate student Karlin Compton for their help in piloting

632 certain experimental conditions. We thank SunyBiotech for their service in generating PHX3914

633 for live imaging experiments. Finally, we thank the Dernburg Lab for providing CA1215, the

634 original dhc-1::degron::GFP worm strain, and Arshad Desai, Federico Pelisch, and Jill

635 Schumacher for antibodies. Some strains were provided by the Caenorhabditis Genetics Center

636 (CGC), which is funded by NIH Office of Research Infrastructure Programs (P40 OD010440).

637 This work was funded by NIH R01GM124354 (to SMW) and by NIH/NCI training grant T32 
bioRxiv preprint doi: https://doi.org/10.1101/2021.09.09.459640; this version posted September 9, 2021. The copyright holder for this preprint

(which was not certified by peer review) is the author/funder, who has granted bioRxiv a license to display the preprint in perpetuity. It is made available under aCC-BY 4.0 International license.

\section{FIGURE 1}

A

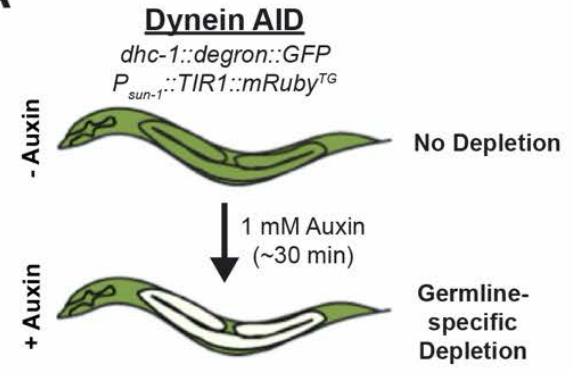

C

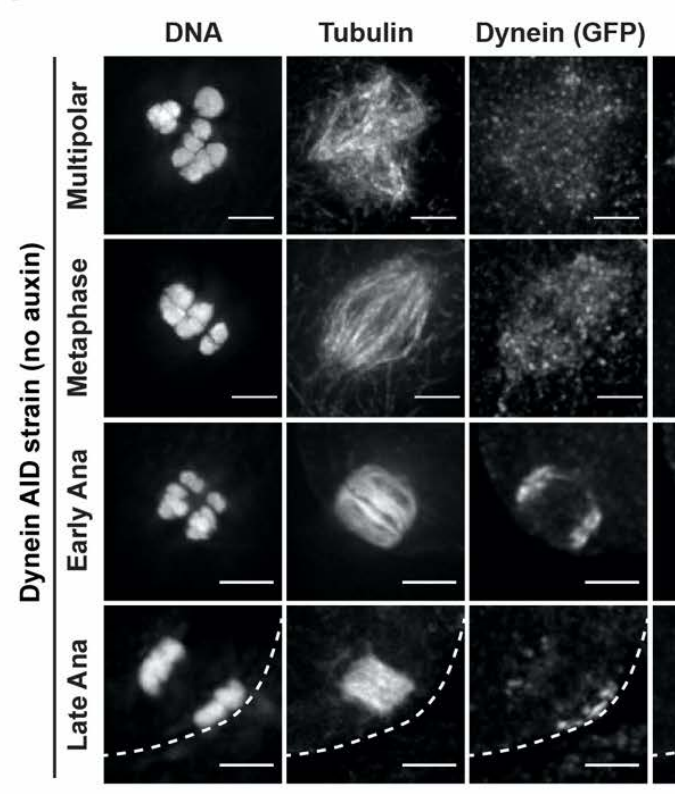

E

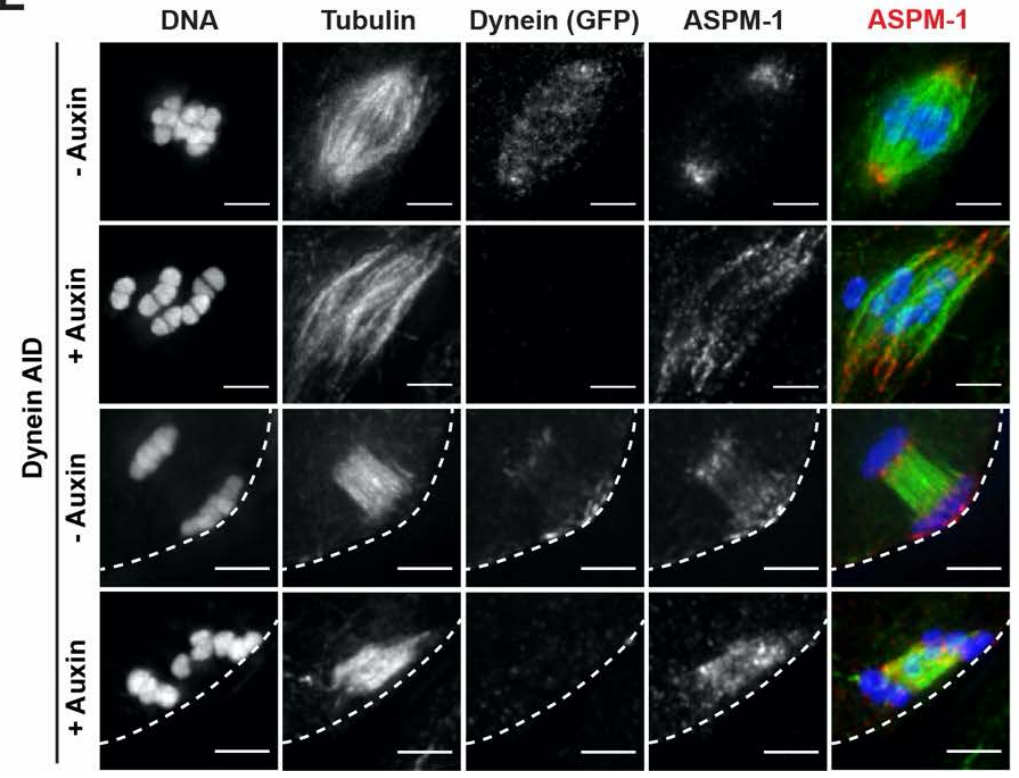

Dynein AID strain

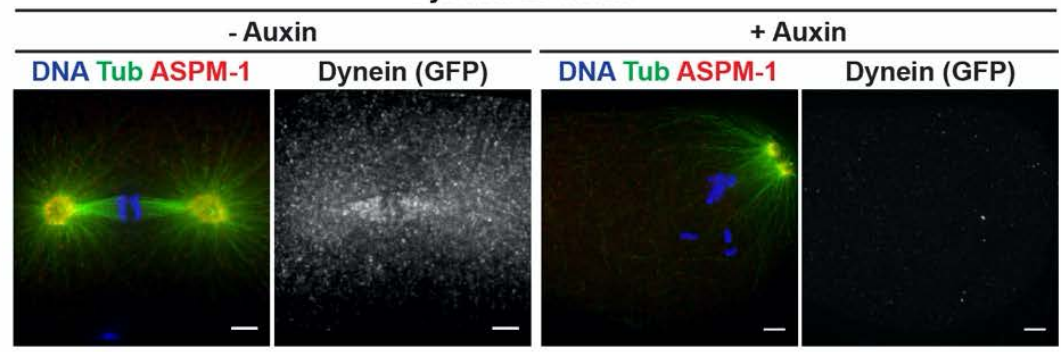

D
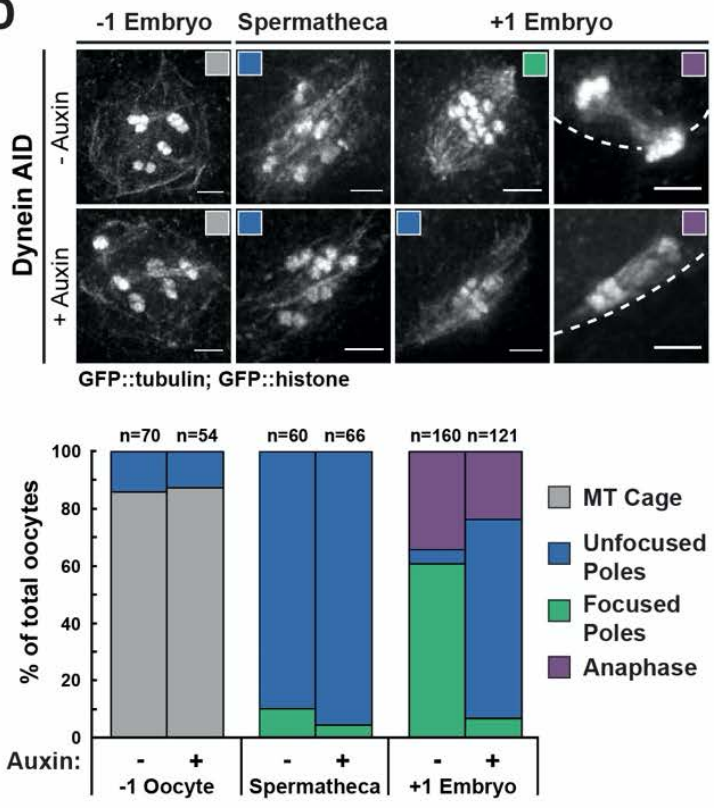

$\mathbf{F}$

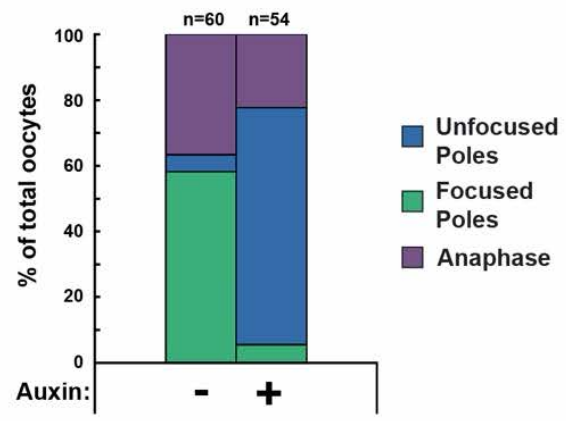


639 Figure 1. Dynein is required for acentrosomal pole focusing.

640 (A) Schematic representation of the Dynein AID system for DHC-1 depletion in the C. elegans

641 germ line. (B) IF imaging of one-cell mitotically-dividing embryos shows that auxin treatment

642 causes efficient dynein depletion and canonical mitotic spindle defects. Shown are tubulin

643 (green), DNA (blue), ASPM-1 (red) and dynein (not shown in merge). (C) IF imaging of oocyte

644 spindles in the Dynein AID strain shows that dynein is localized to the spindle, with increasing

645 enrichment at acentrosomal poles at the anaphase transition; shown are tubulin (green), DNA

646 (blue), dynein (red) and ASPM-1 (not shown in merge). Cortex is represented by dashed line.

647 (D) Representative images of oocyte spindles (GFP::tubulin and GFP::histone) in germline

648 counting and corresponding quantifications; auxin treatment leads to splayed poles and spindle

649 rotation defects. Cortex is represented by dashed line. (E) IF imaging of Dynein AID conditions

650 showing effects of 4-hour auxin treatment on metaphase (second row) and anaphase (bottom

651 row); shown are tubulin (green), DNA (blue), ASPM-1 (red) and dynein (not shown in merge).

652 ASPM-1 labeling supports initial observations of splayed poles seen in germline counting. (F)

653 Quantifications of IF imaging shown in (E); meiotic spindles have significantly splayed poles

654 upon auxin treatment. All scale bars $=2.5 \mu \mathrm{m}$. 
bioRxiv preprint doi: https://doi.org/10.1101/2021.09.09.459640; this version posted September 9, 2021. The copyright holder for this preprint

(which was not certified by peer review) is the author/funder, who has granted bioRxiv a license to display the preprint in perpetuity. It is made available under aCC-BY 4.0 International license.

\section{FIGURE 2}

A

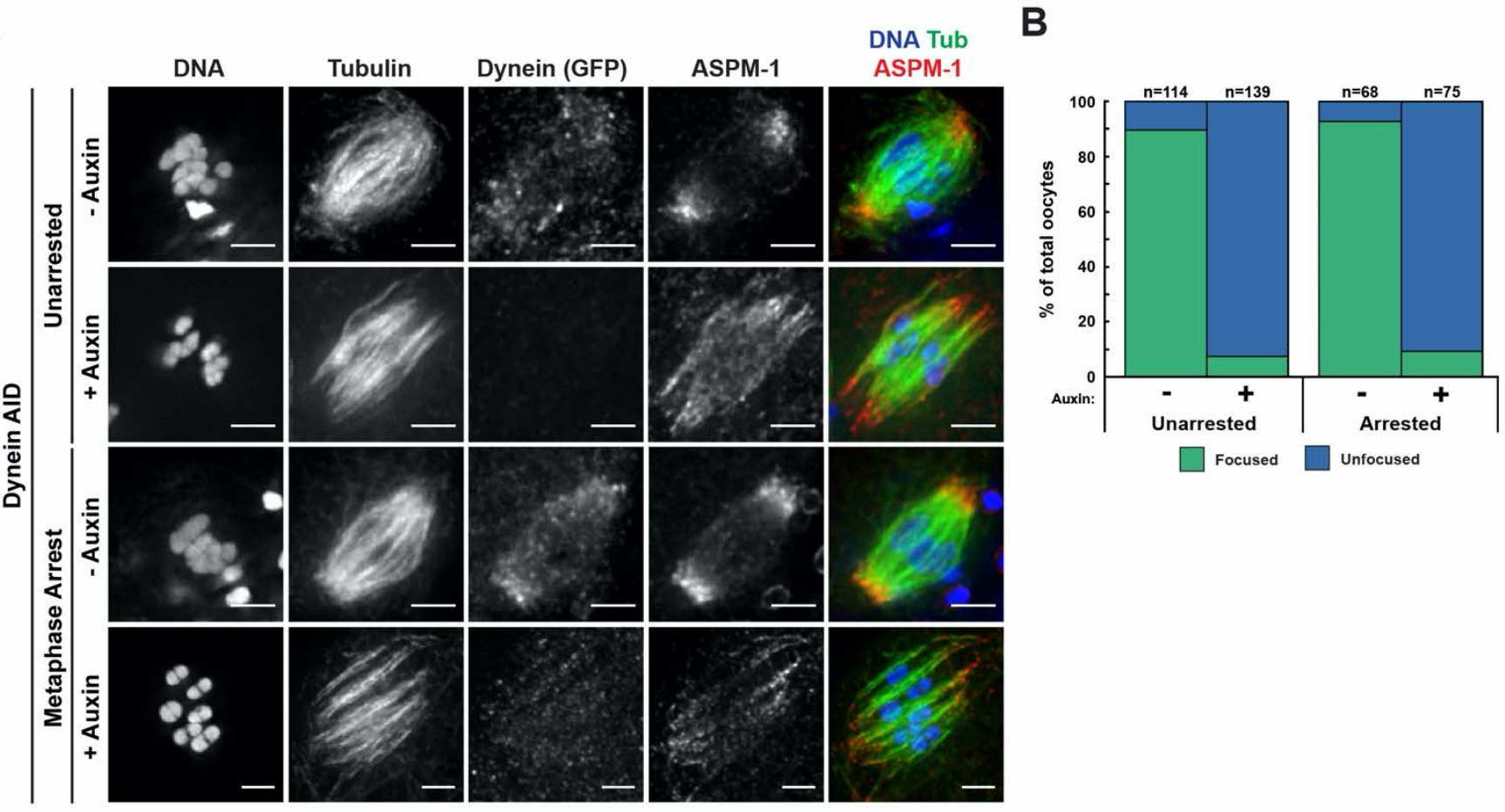

C
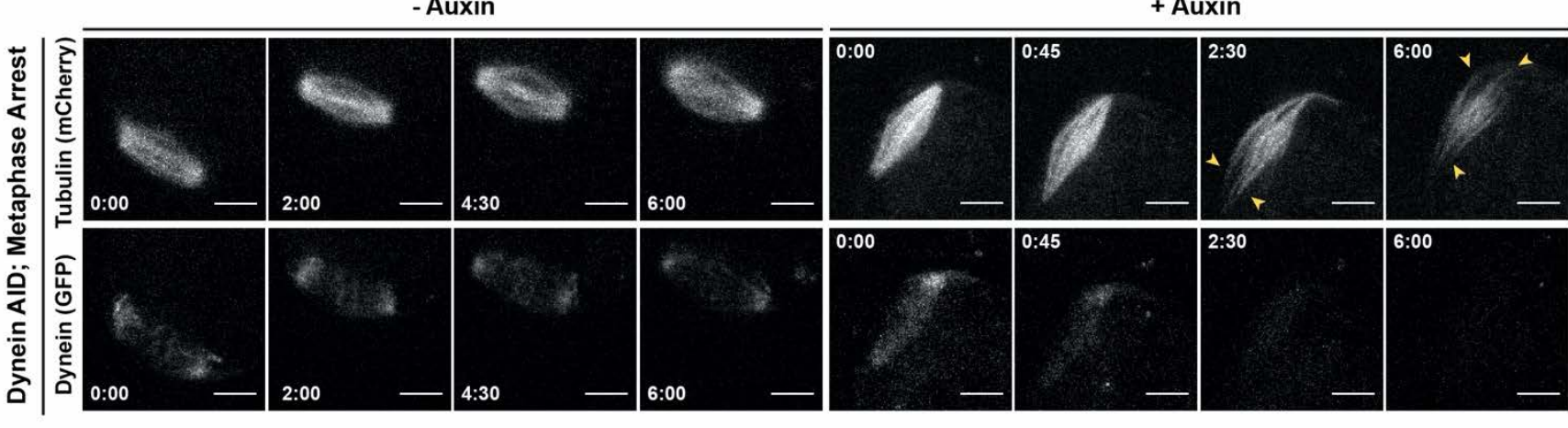

D

GFP::tubulin; GFP::histone

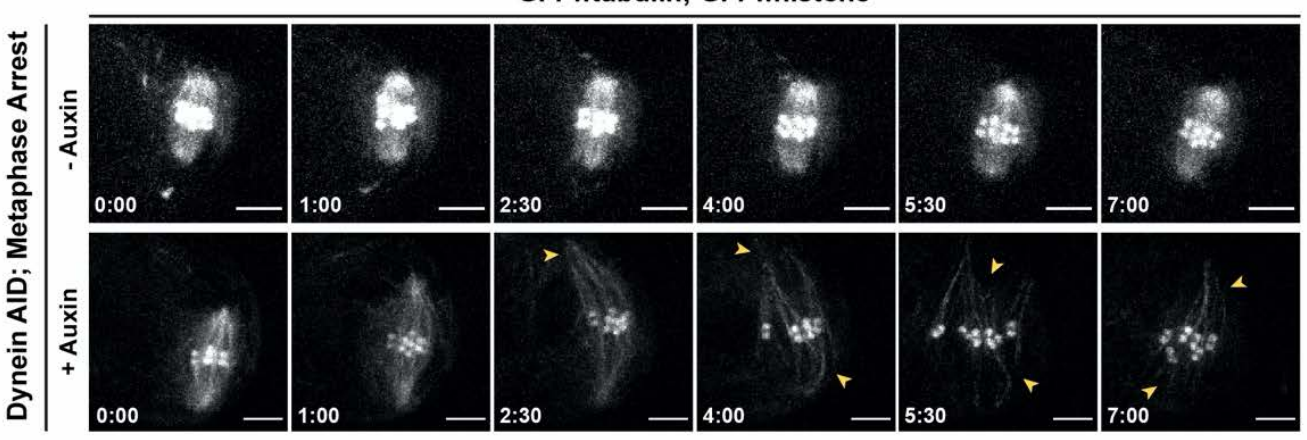


655 Figure 2. Dynein is required to maintain focused spindle poles.

656 (A) IF imaging of oocyte spindles in control or metaphase-arrest (emb-30(RNAi)) conditions;

657 shown are tubulin (green), DNA (blue), ASPM-1 (red) and dynein (not shown in merge).

658 Acentrosomal poles become unfocused upon acute dynein depletion. Scale bars $=2.5 \mu \mathrm{m}$. $(B)$

659 Quantifications of bipolar spindles imaged in $(A)$ shows that addition of auxin causes nearly all

660 spindle poles to unfocus and splay, both with and without the metaphase arrest. Further

661 quantification of changes in spindle length and microtubule minus end distribution upon auxin

662 treatment can be seen in Figure Supplement 1A and 1B. (C) ex utero live imaging of

663 metaphase-arrested spindles; vehicle-treated control oocytes are shown on the left, auxin-

664 treated oocytes are shown on the right. Addition of auxin causes rapid depletion of dynein

665 (labeled with GFP, bottom row) and dynamic splaying of both spindle poles (shown using

666 mCherry::tubulin, top row; arrowheads). Time elapsed shown in (min):(sec). Scale bars $=5 \mu \mathrm{m}$.

667 (D) ex utero live imaging of metaphase-arrested spindles, shown using GFP::tubulin and

668 GFP::histone; addition of auxin (bottom row) causes dynamic splaying of poles (arrowheads)

669 but does not grossly disrupt chromosome alignment; vehicle-treated control oocytes are shown

670 on the top row. Time elapsed shown in $(\mathrm{min}):(\mathrm{sec})$. Scale bars $=5 \mu \mathrm{m}$. Additional ex utero

671 imaging of unarrested embryos treated with auxin can be seen in Figure Supplement 2. 
bioRxiv preprint doi: https://doi.org/10.1101/2021.09.09.459640; this version posted September 9, 2021. The copyright holder for this preprint (which was not certified by peer review) is the author/funder, who has granted bioRxiv a license to display the preprint in perpetuity. It is made available under aCC-BY 4.0 International license.

\section{FIGURE 2 - figure supplement 1}

A
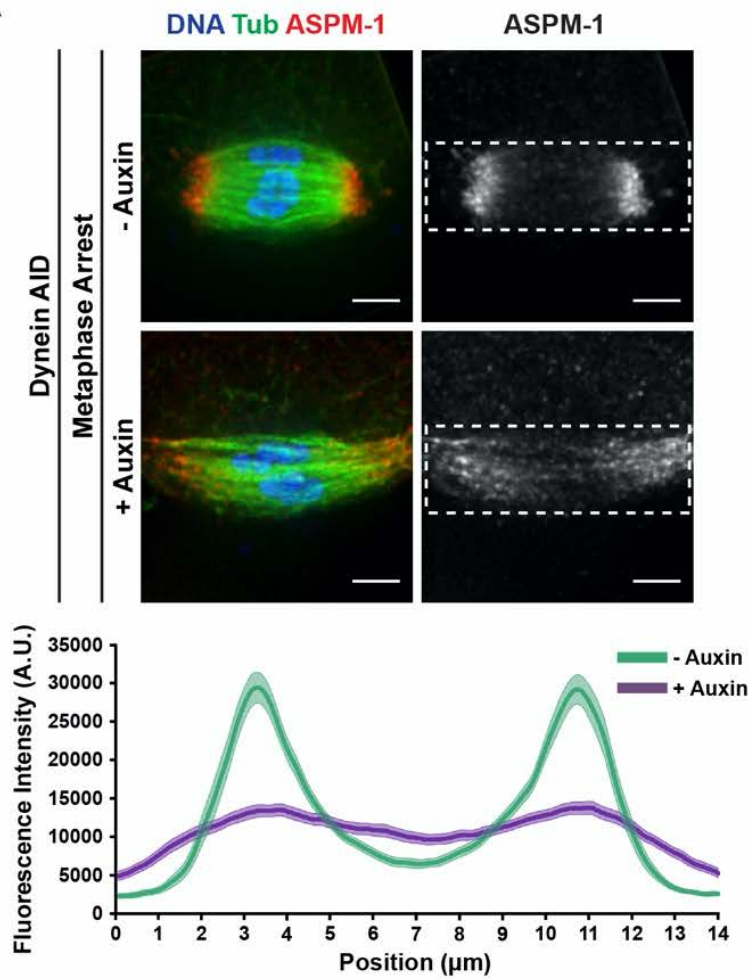

Arrested Spindle Length After Auxin Treatment

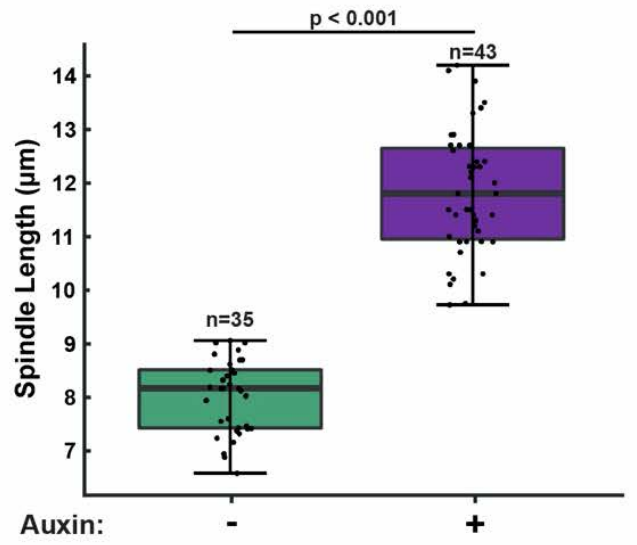

B
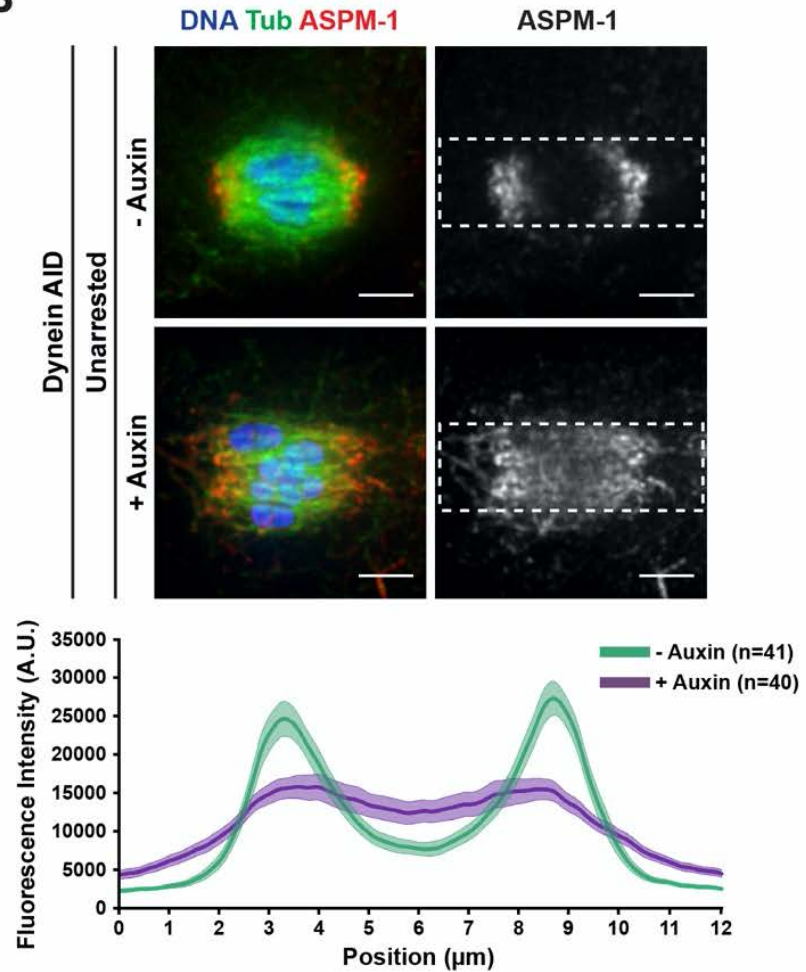

Unarrested Spindle Length After Auxin Treatment

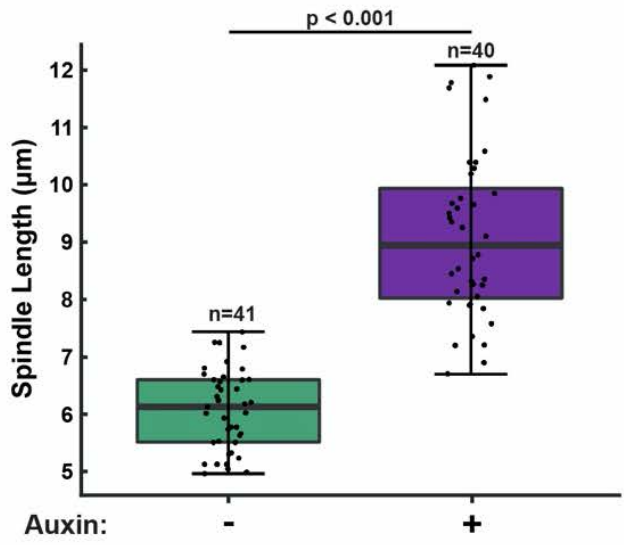


672 Figure 2 - figure supplement 1. Acute dynein depletion leads to dispersion of ASPM-1

673 across the spindle and increased pole-to-pole spindle length.

674 Representative IF images showing the phenotype of acute dynein depletion from metaphase-

675 arrested (emb-30(RNAi)) spindles (A) or control spindles (B) in Dynein AID worms; shown are

676 microtubules (green), DNA (blue), and ASPM-1 (red). Dashed rectangles represent ROI used

677 for linescan measurements ( $4 \mu \mathrm{m}$ tall by 12 or $14 \mu \mathrm{m}$ wide). Quantifications of fluorescence

678 intensity across the length of the spindle demonstrate that dynein depletion leads to

679 considerably more uniform and dispersed ASPM-1 localization. All images used for linescan

680 analysis were also measured for pole-to-pole spindle length, and these measurements were

681 plotted in corresponding boxplots. Upon addition of auxin, the spindle is significantly longer. All

682 scale bars $=2.5 \mu \mathrm{m}$. Statistical significance between means of spindle length was determined

683 via a two-tailed $t$ test for unarrested and arrested conditions, with a $p$ value of $p<2.2 \times 10^{-16}$ for

684 both. 
FIGURE 2 - figure supplement 2

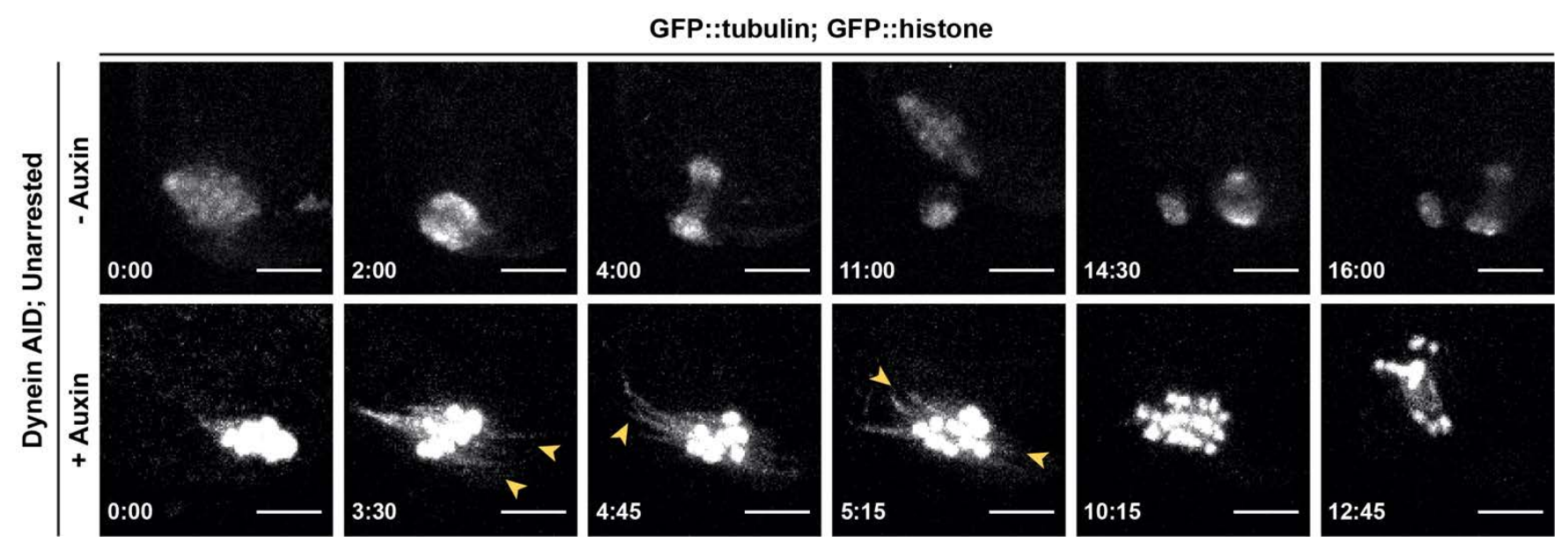

685 Figure 2 - figure supplement 2. Phenotypes seen in dynein depletion are not an artifact

686 of metaphase arrest.

687 Repeat of ex utero live imaging in Figure 2D, but with unarrested embryos. Splaying of spindle

688 poles (arrowheads) is identical to splaying observed in metaphase-arrested spindles (Figure

689 2C, 2D). We also observed spindle rotation defects in anaphase, consistent with prior studies.

690 Time elapsed shown in $(\mathrm{min}):(\mathrm{sec})$. Scale bars $=5 \mu \mathrm{m}$. 


\section{FIGURE 3}

A

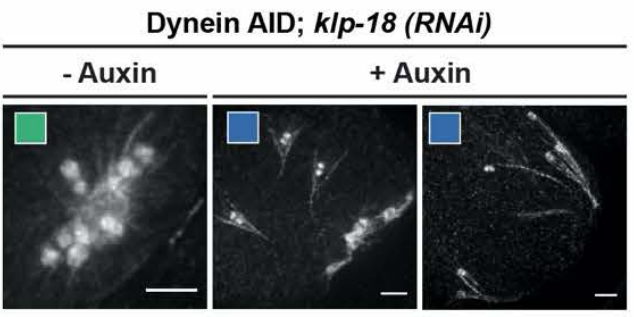

GFP::tubulin; GFP::histone

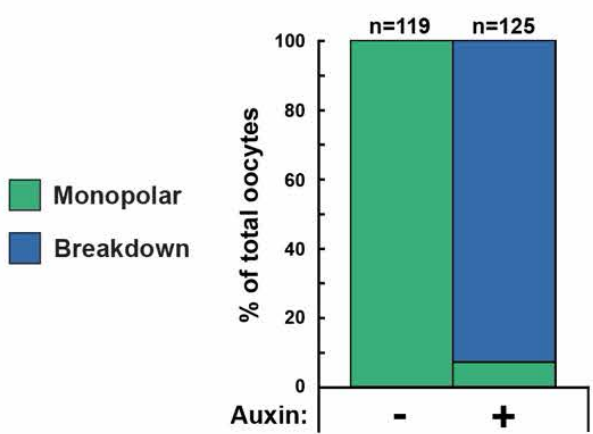

B

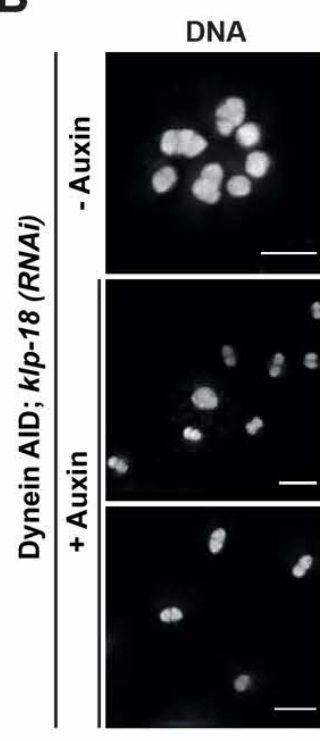

C
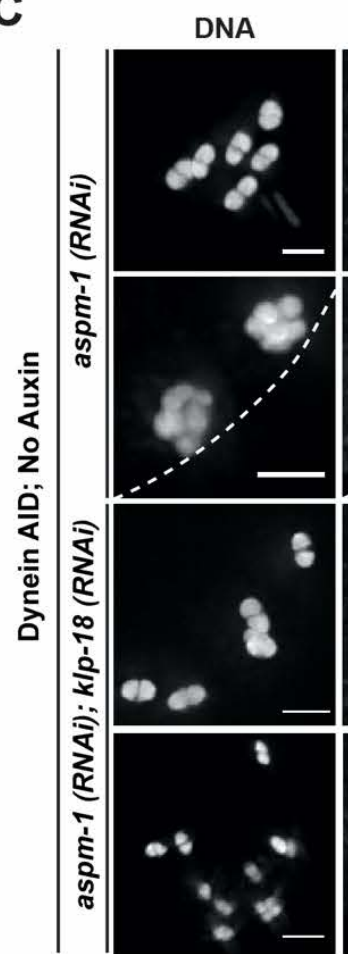

Tubulin

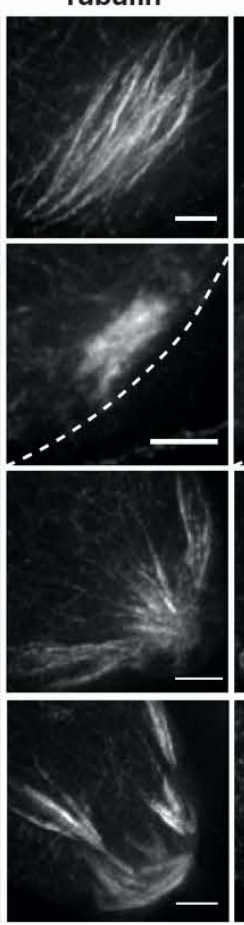

Dynein (GFP)

ASPM-1

DNA Tub

ASPM-1

DNA Tub ASPM-1
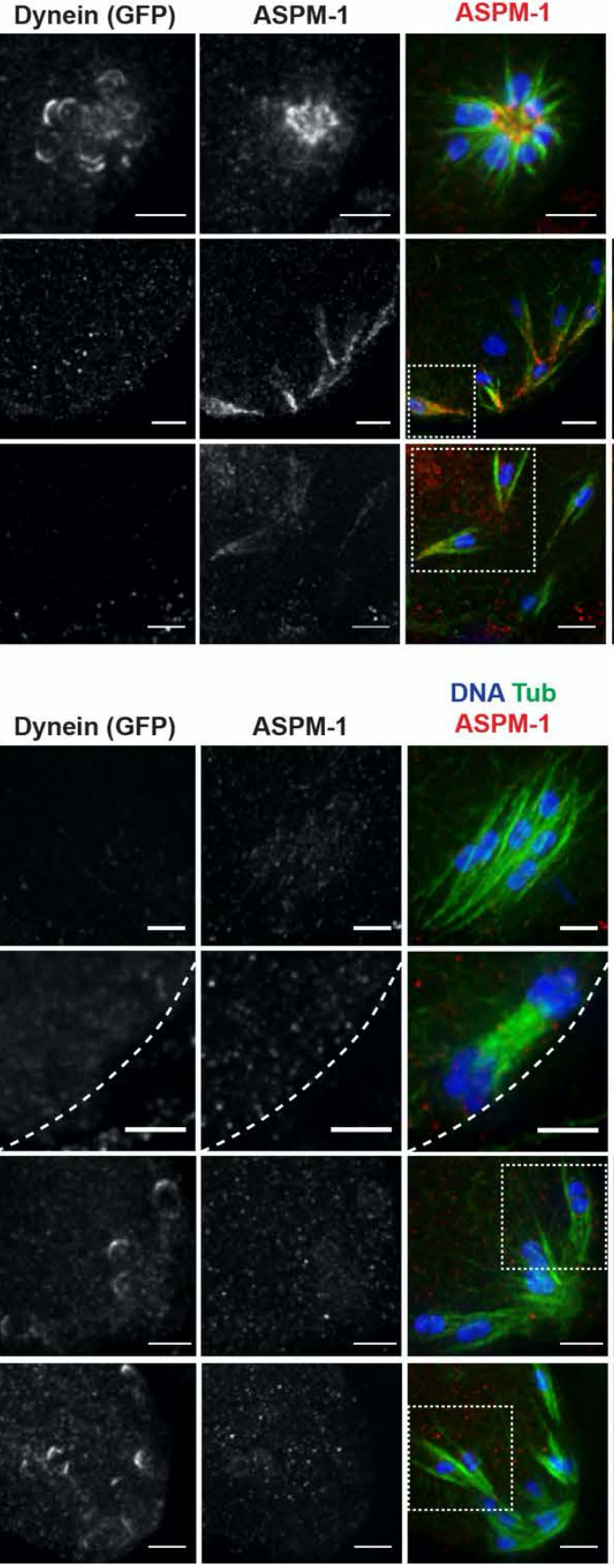

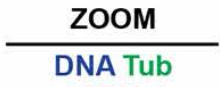
ASPM-1
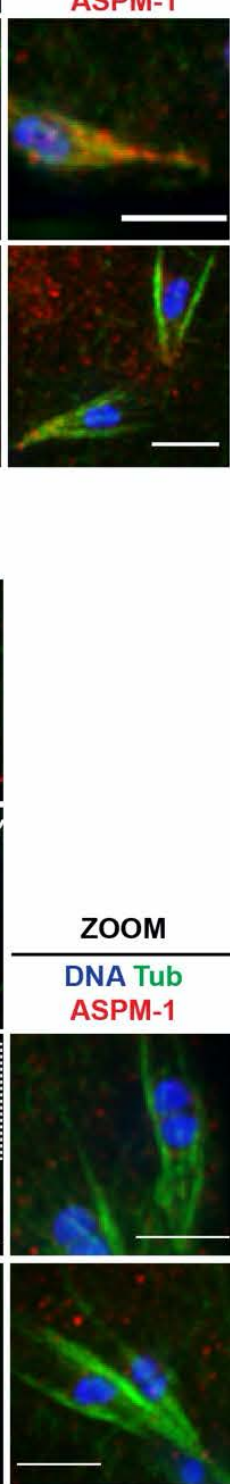
691 Figure 3. Monopolar spindles breakdown upon dynein depletion.

692 (A) Representative images of klp-18(RNAi) oocyte spindles (GFP::tubulin and GFP::histone) in

693 germline counting and corresponding quantifications; dynein depletion leads to dissolution of

694 monopole, releasing chromosomes with associated microtubule bundles into the cytoplasm. (B)

695 IF imaging of monopole breakdown after dynein depletion; shown are tubulin (green), DNA

696 (blue), ASPM-1 (red) and dynein (not shown in merge). Chromosomes released into the

697 cytoplasm retain lateral microtubule associations (zooms). (C) IF imaging of Dynein AID

698 embryos following either aspm-1 single RNAi or aspm-1; klp-18 double RNAi; shown are tubulin

699 (green), DNA (blue), ASPM-1 (red) and dynein (not shown in merge). Bipolar spindles exhibit

700 significant splaying of spindle poles and spindle rotation defects in anaphase (top two rows).

701 Double RNAi causes partial breakdown of monopolar spindles (bottom two rows). Cortex

702 represented by dashed line. All scale bars $=2.5 \mu \mathrm{m}$. Additional conditions involving lin-5(RNAi)

703 can be seen in Figure Supplement 1A and 1B. 
FIGURE 3 - figure supplement 1

A

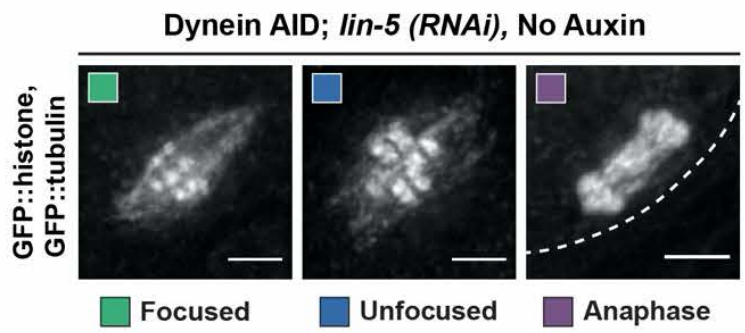

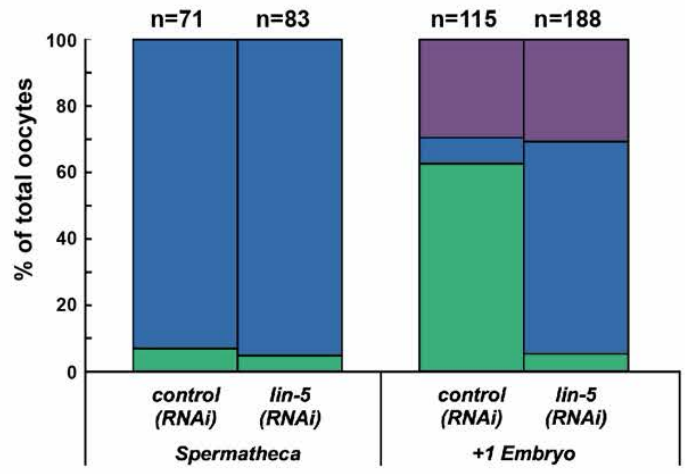

B

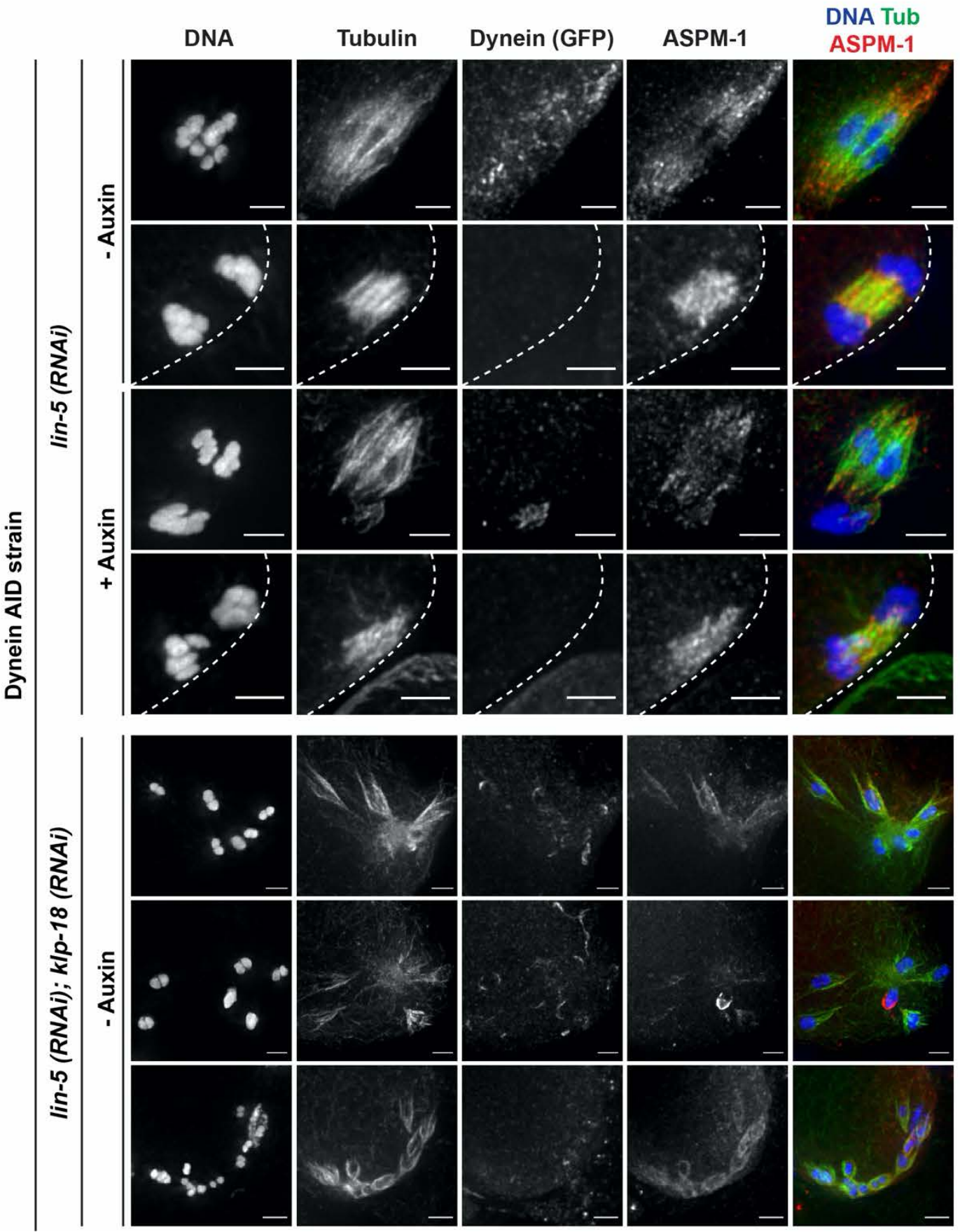


704 Figure 3 - figure supplement 1. Meiotic spindles following LIN-5 depletion are 705 morphologically similar to Dynein AID conditions.

706 A) Representative images of spindles counted in germline imaging from the Dynein AID strain 707 expressing GFP::tubulin and GFP::histone. In lin-5(RNAi) conditions, splaying of acentrosomal 708 poles can be observed in the +1 position. We also observed improper spindle rotation during 709 anaphase, consistent with prior studies. Quantifications demonstrated similar ratios of 710 unfocused poles as those seen in Dynein AID germline counting. Cortex represented by dashed 711 line. B) IF imaging of embryos from Dynein AID worms in lin-5 RNAi conditions; shown are 712 tubulin (green), DNA (blue), ASPM-1 (red) and dynein (not shown in merge). ASPM-1 labeling

713 further supports splaying of acentrosomal poles and improper spindle rotation during anaphase

714 can also be seen. Concurrent acute dynein depletion (rows 3, 4) does not seem to exacerbate

715 the spindle defects observed in lin-5(RNAi) alone. Double RNAi depletions with klp-18(RNAi)

716 cause similar breakdown phenotypes to Dynein AID depletions on monopoles (rows 5, 6, 7).

717 Cortex represented by dashed line. All scale bars $=2.5 \mu \mathrm{m}$. 


\section{FIGURE 4}

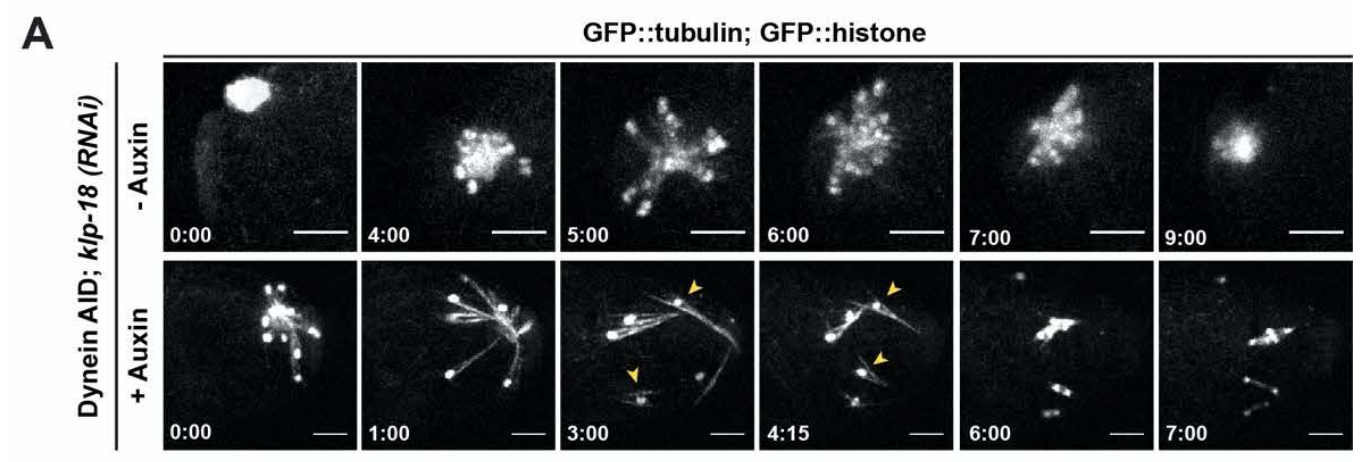

B

GFP::tubulin; GFP::histone

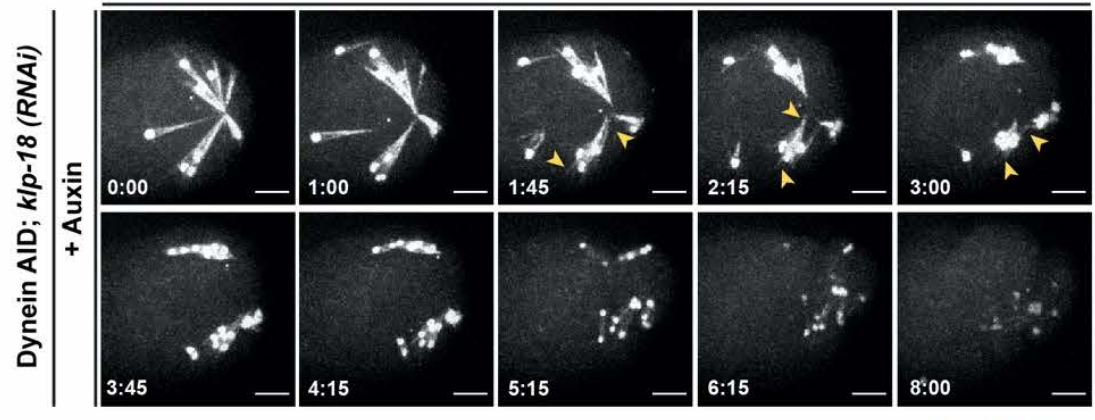

C

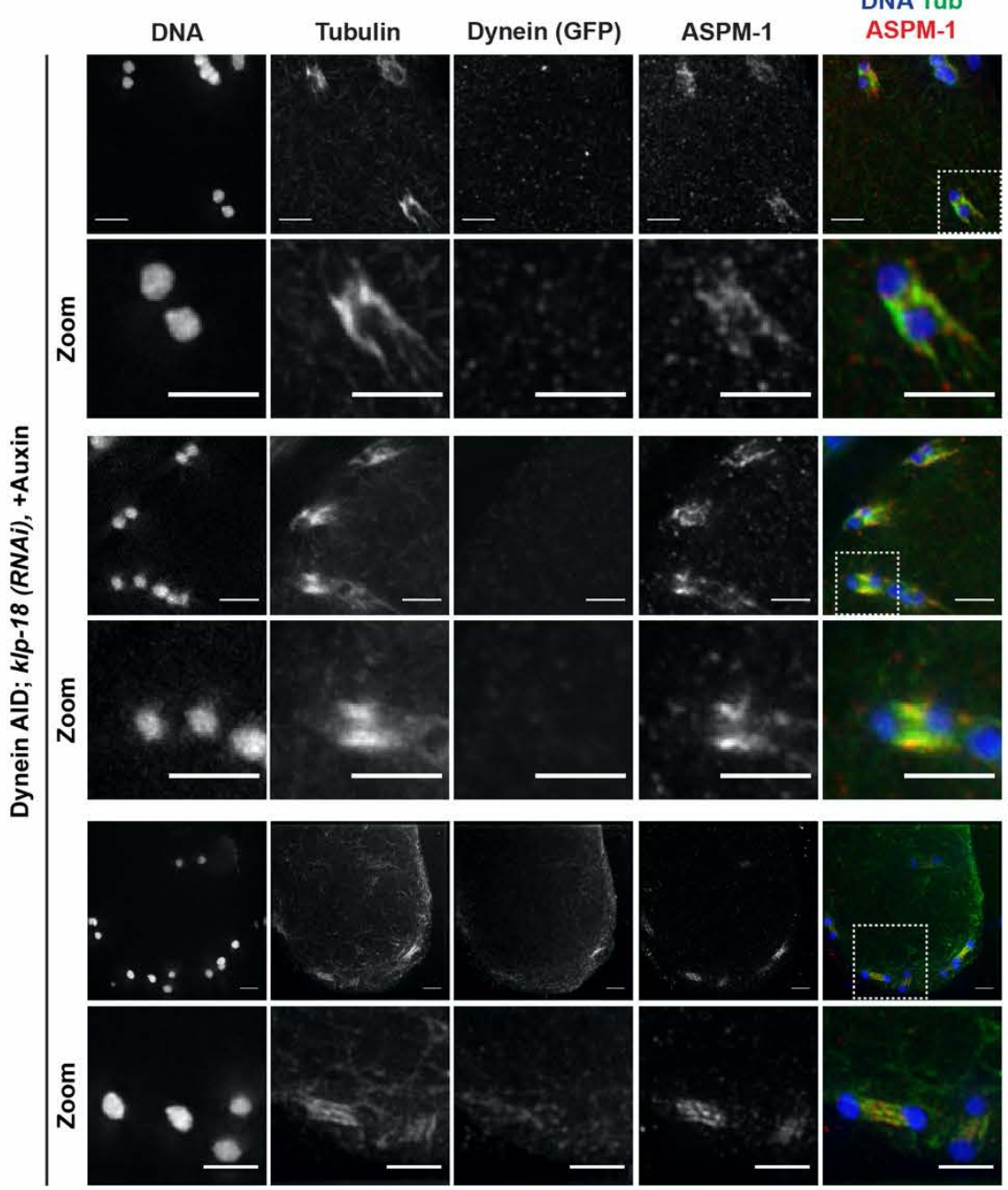

Early

Anaphase

Mid

Anaphase

Late Anaphase 
718 Figure 4. Individualized chromosomes undergo anaphase-like segregation in the absence 719 of KLP-18 and dynein.

720 (A) ex utero live imaging of GFP::tubulin and GFP::histone in control and klp-18(RNAi)

721 conditions. In the control (top row), a monopolar spindle forms and then chromosomes move

722 back towards the monopole in anaphase, as previously described (Muscat et al., 2015). Addition

723 of auxin to acutely deplete dynein (bottom row) leads to rapid breakdown of monopolar spindles.

724 Microtubule bundles remain laterally associated with chromosomes (arrowheads) prior to

725 anaphase-like segregation. Time elapsed shown in ( $\min )$ :(sec). Scale bars $=5 \mu \mathrm{m}$. (B) Another

726 example of acute auxin treatment, to remove dynein, in klp-18(RNAi) conditions; after

727 breakdown of the monopole and reorganization of microtubules (arrowheads), individual

728 bivalents are able to undergo synchronized anaphase-like segregation. Time elapsed shown in

729 ( $\mathrm{min})$ :(sec). Scale bars $=5 \mu \mathrm{m}$. (C) IF imaging of miniature anaphases at multiple chromosome

730 segregation distances, representing various stages of anaphase; shown are tubulin (green),

731 DNA (blue), ASPM-1 (red) and dynein (not shown in merge). Segregation events closely

732 resemble different stages of anaphase $A$ and anaphase B. Scale bars $=2.5 \mu \mathrm{m}$. Quantifications

733 of miniature anaphase segregation distances and rates can be seen in Figure Supplement 1. 


\section{FIGURE 4 - figure supplement 1}

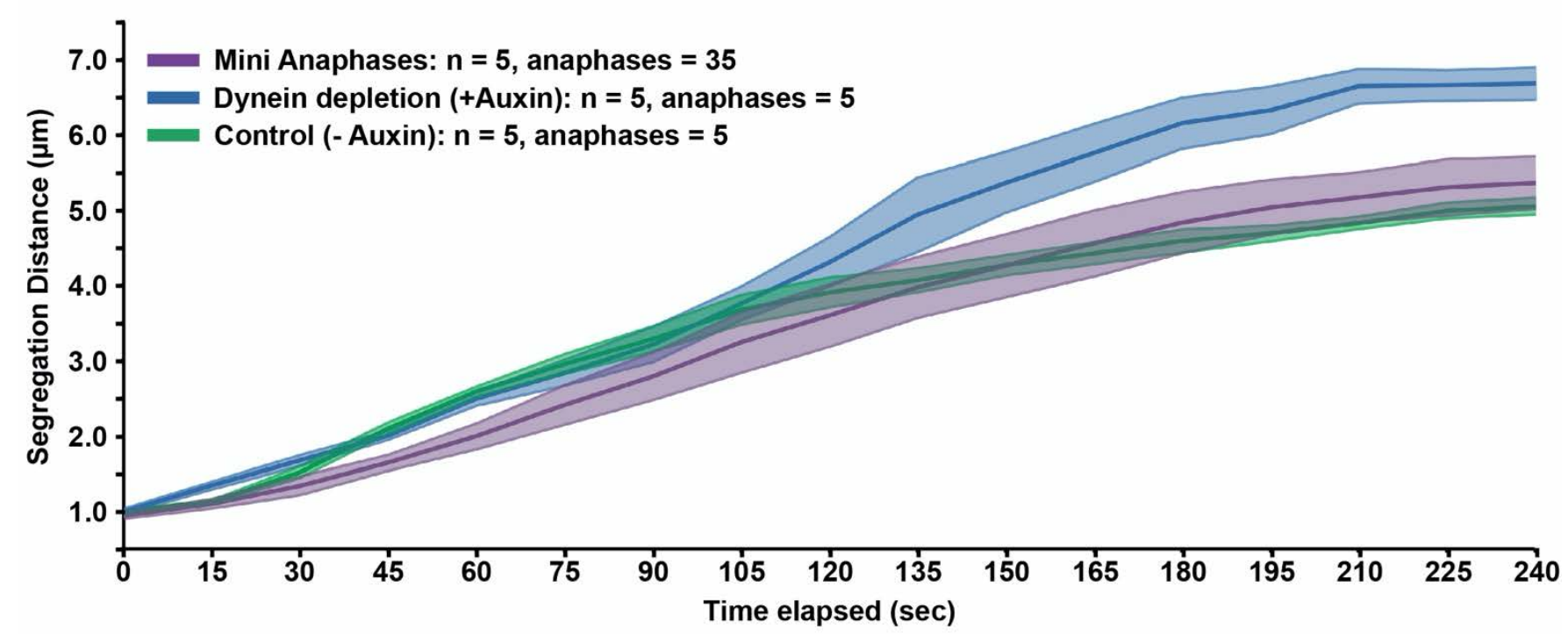

Figure 4 - figure supplement 1. Miniature anaphases segregate chromosomes at distances and rates comparable to normal anaphase spindles.

736 Quantification of timelapses from control anaphases (the Dynein AID strain without auxin),

737 anaphases in the absence of dynein (the Dynein AID strain with auxin), and miniature

738 anaphases. Each timelapse was synchronized to initiation of anaphase A, and the distance

739 between the centers of each chromosome was measured at each 15 second timepoint. For

740 each condition, the number of timelapses used is represented by $n$, and the shaded area

741 around each average line represents SEM for each condition. Note that for the mini anaphase

742 condition, though we used 5 movies, we measured multiple mini anaphases in each (total

743 anaphases $=35$ ). Miniature anaphases do not exhibit significant differences in segregation rates

744 or distances to wild type anaphase spindles, but do not reach distances of dynein depletions

745 alone. 


\section{FIGURE 5}

A

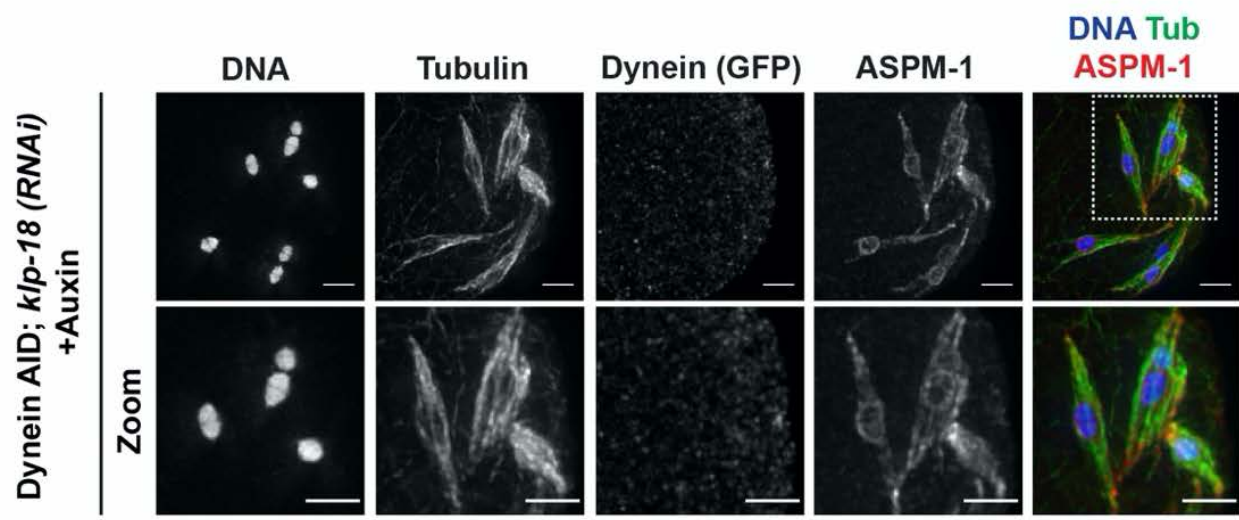

B

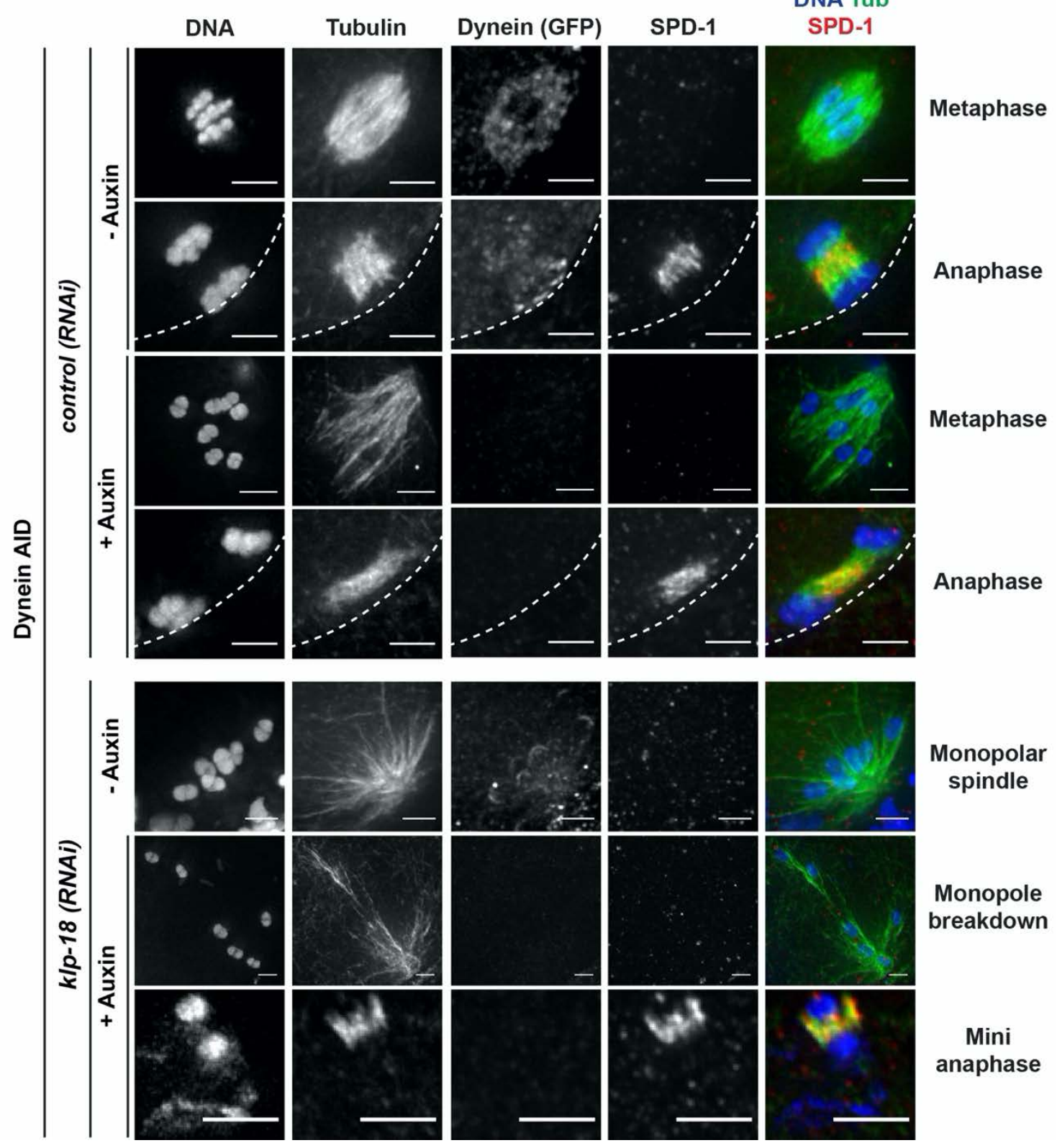


746 Figure 5. Microtubules reorganize into miniature bipolar spindles that can segregate

747 chromosomes.

748 (A) IF imaging of microtubules (green), DNA (blue), ASPM-1 (red) and dynein (not shown in

749 merge) in monopolar spindle breakdown conditions (klp-18(RNAi) + acute dynein AID).

750 Microtubule bundles appear to reorganize around individual chromosomes, seen through

751 ASPM-1 flanking either side of the chromosome; note that in these images ASPM-1 also

752 appears to be on chromosomes, but that is background staining that sometimes occurs with this

753 antibody (Wignall and Villeneuve, 2009) and is not real signal. (B) IF imaging of SPD-1

754 localization in the Dynein AID strain in control RNAi conditions (rows 1-4) or following klp-

755 18(RNAi) (rows 5-7); shown are tubulin (green), DNA (blue), SPD-1 (red) and dynein (not shown

756 in merge). SPD-1 does not localize to spindles in metaphase (rows 1, 3), but localizes to

757 overlapping microtubules in anaphase spindles in the presence or absence of dynein (rows 2 ,

758 4). SPD-1 is not localized to monopolar spindles either before or after monopole breakdown

759 (rows 5,6 ), but can clearly be seen localized to miniature anaphases (row 7). Cortex is

760 represented by dashed line. All scale bars $=2.5 \mu \mathrm{m}$. Additional observations of SUMO

761 localization can be seen in Figure Supplement 1. 


\section{FIGURE 5 - figure supplement 1}

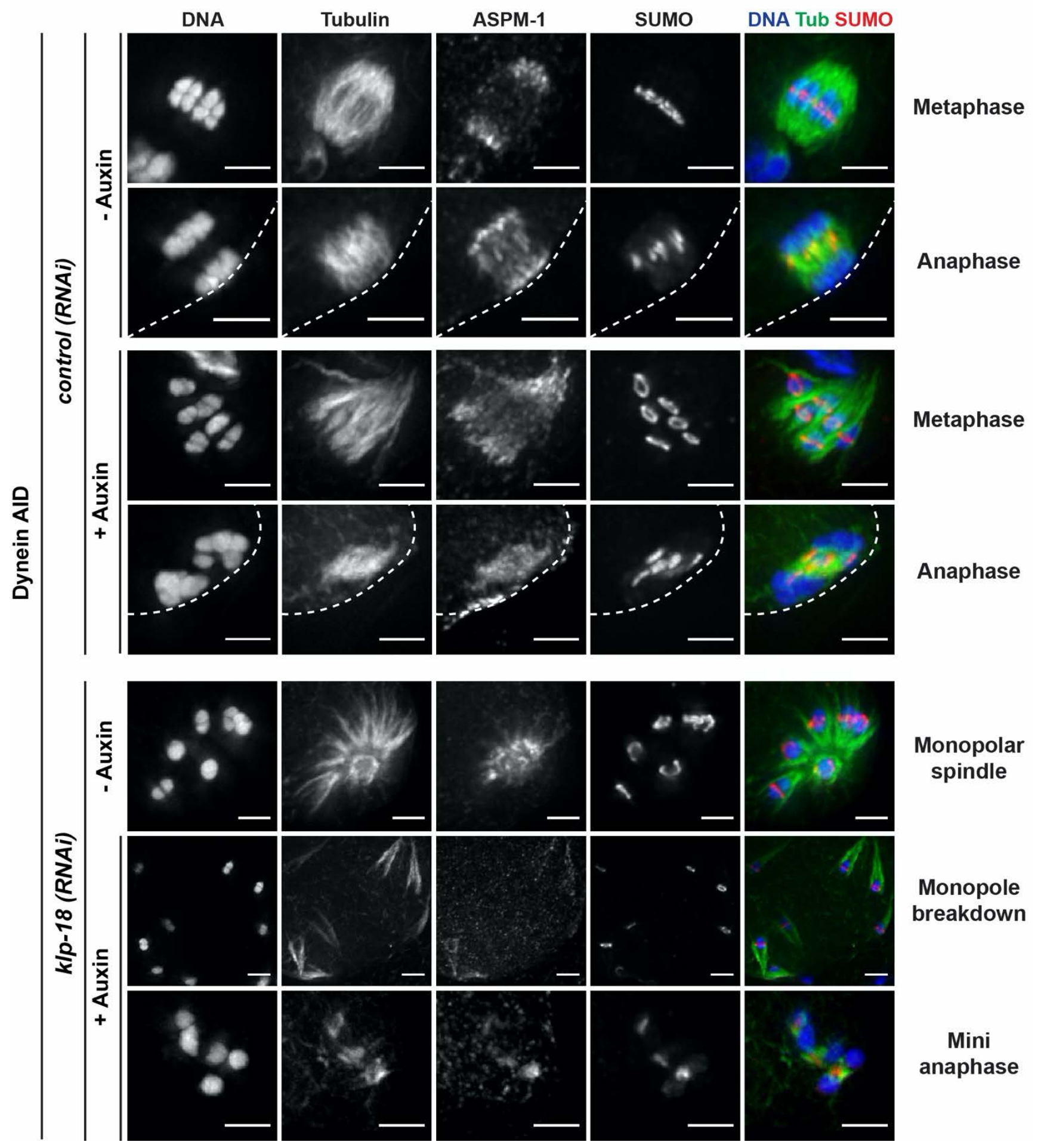


762 Figure 5 - figure supplement 1. SUMO is localized normally in all dynein depletion

763 conditions.

764 IF imaging of control and klp-18(RNAi) conditions; shown are tubulin (green), DNA (blue),

765 SUMO (red) and ASPM-1 (not shown in merge). SUMO is localized to a ring complex that forms

766 around chromosomes and then is released from chromosomes in anaphase in the presence or

767 absence of dynein (rows 1-4). SUMO remains localized to the ring complex before and after

768 monopole breakdown (rows 5,6), and can clearly be seen localized between separating

769 chromosomes in miniature anaphases (row 7). Cortex is represented by dashed line. Scale bars

$770=2.5 \mu \mathrm{m}$. 
bioRxiv preprint doi: https://doi.org/10.1101/2021.09.09.459640; this version posted September 9, 2021. The copyright holder for this preprint

(which was not certified by peer review) is the author/funder, who has granted bioRxiv a license to display the preprint in perpetuity. It is made available under aCC-BY 4.0 International license.

\section{FIGURE 6}
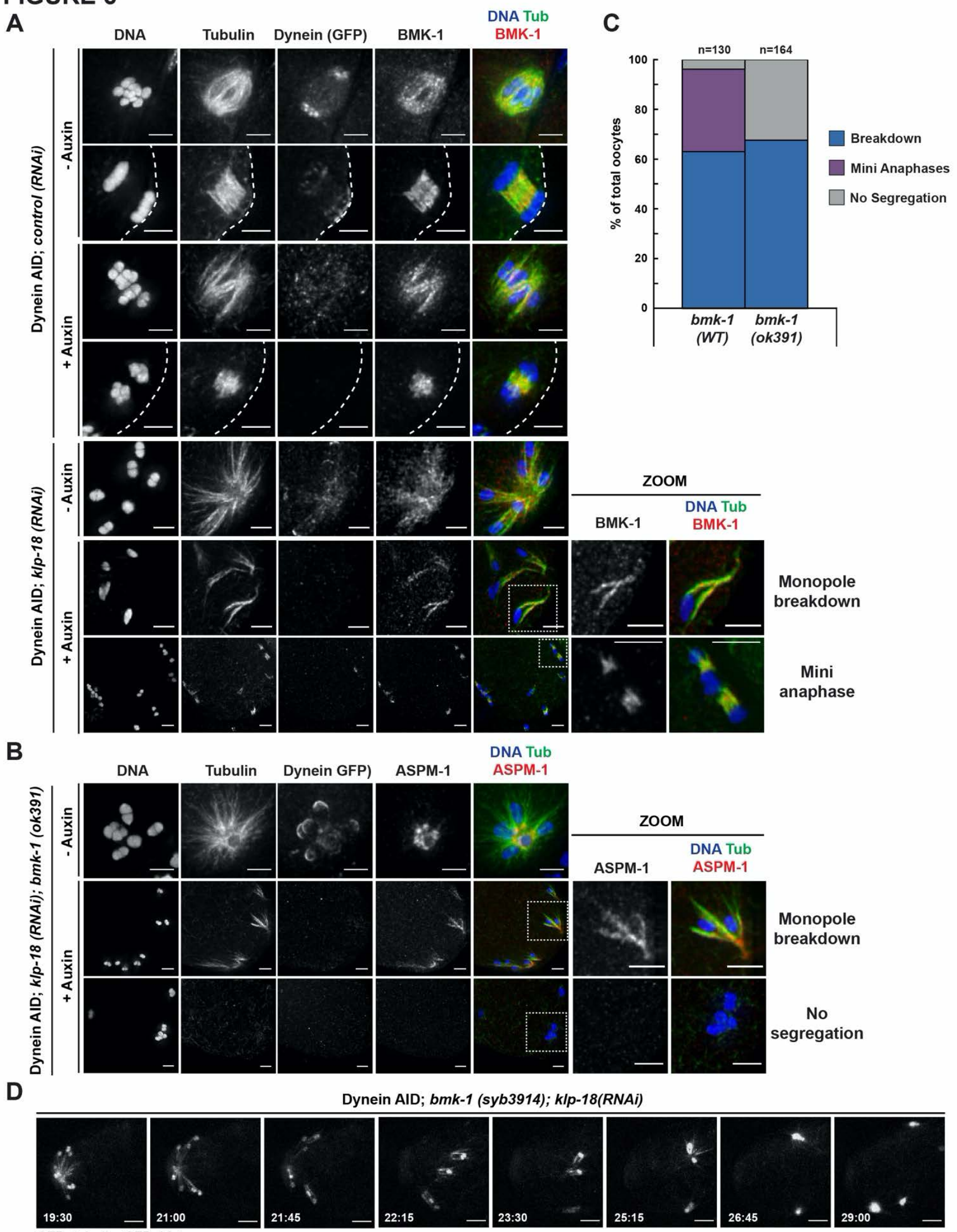
771 Figure 6. BMK-1 localizes to the meiotic spindle and is required for microtubule 772 reorganization and the formation of miniature anaphases.

773 (A) IF imaging of embryos in either control or klp-18(RNAi) conditions in the Dynein AID strain in

774 the presence and absence of acute auxin treatment; shown are tubulin (green), DNA (blue),

775 BMK-1 (red) and dynein (not shown in merge). BMK-1 is localized to spindle microtubules in all

776 conditions, including following monopolar spindle breakdown and in mini anaphases (zoomed

777 images). Cortex is represented by dashed line. Scale bars $=2.5 \mu \mathrm{m}$. (B) IF imaging of embryos

778 following klp-18(RNAi) in the Dynein AID strain lacking functional BMK-1 (bmk-1(ok391)); shown

779 are tubulin (green), DNA (blue), ASPM-1 (red) and dynein (not shown in merge). Following

780 monopolar spindle breakdown in the presence of auxin (rows 2, 3), embryos do not contain

781 miniature anaphases and lack chromosome segregation. Scale bars $=2.5 \mu \mathrm{m}$. (C)

782 Quantifications of images shown in (B) compared to wild type (WT) embryos; monopolar

783 spindles still break down but no miniature anaphases are observed in embryos lacking BMK-1

784 function. (D) ex utero live imaging of GFP::tubulin and GFP::histone following acute auxin

785 treatment to remove dynein in klp-18(RNAi); bmk-1(syb3914) conditions; miniature anaphases

786 do not form in the absence of BMK-1. Time elapsed shown in $(\mathrm{min})$ :(sec). Scale bars $=5 \mu \mathrm{m}$.

787 Validation of $b m k-1$ mutants via IF imaging can be seen in Figure Supplement 1. 
FIGURE 6 - figure supplement 1

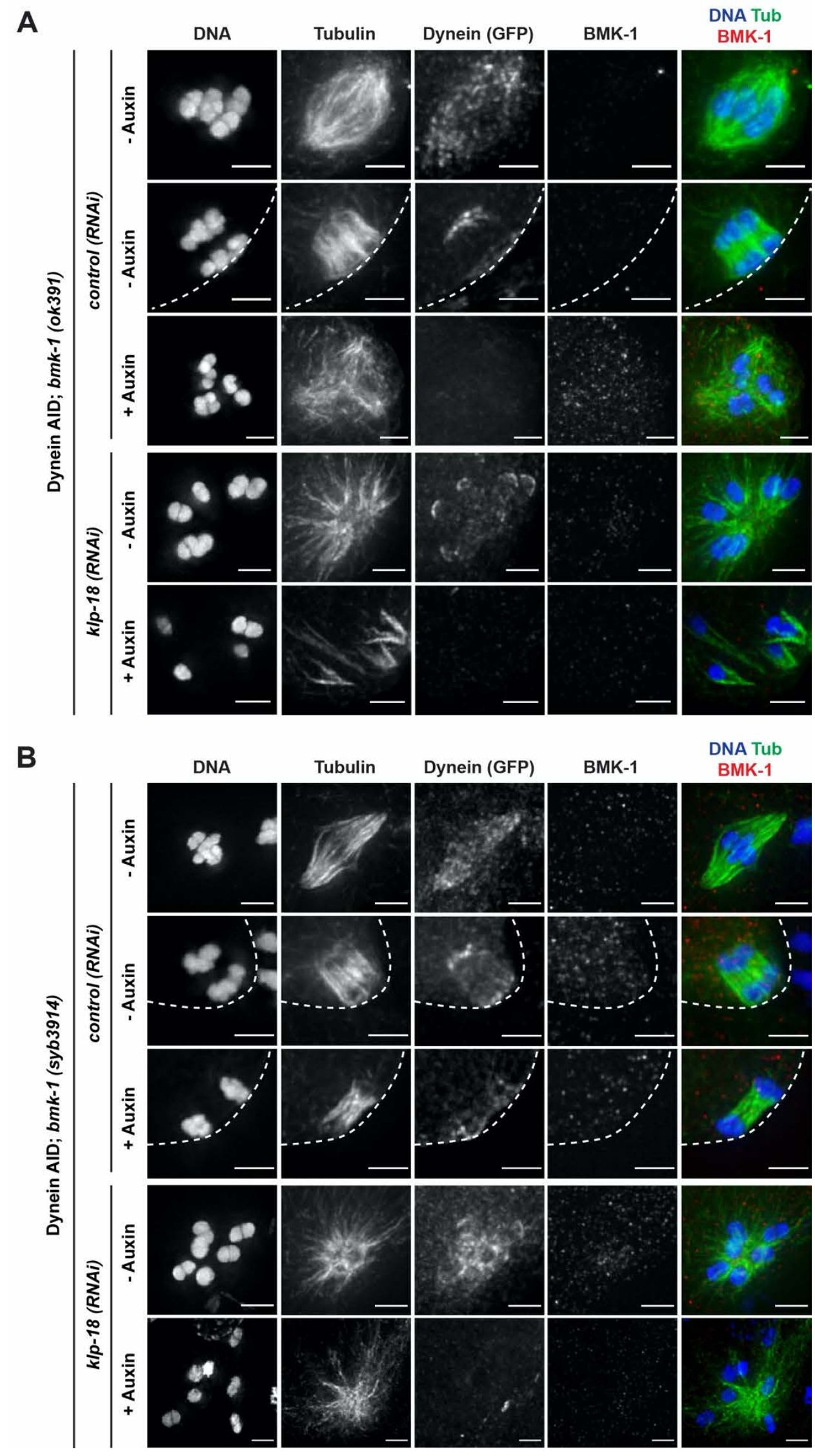


788 Figure 6 - figure supplement 1. IF imaging validation of BMK-1 deletion in worm strains.

789 IF imaging of embryos containing either the bmk-1(ok391) deletion allele (A) or the bmk-

790 1(syb3914) deletion allele (B); shown are tubulin (green), DNA (blue), BMK-1 (red) and dynein

791 (not shown in merge). No BMK-1 localization can be observed in either mutant in control(RNAi)

792 or klp-18(RNAi) conditions in the presence or absence of auxin. Cortex represented by dashed

793 line. Scale bars $=2.5 \mu \mathrm{m}$. 


\section{FIGURE 7}

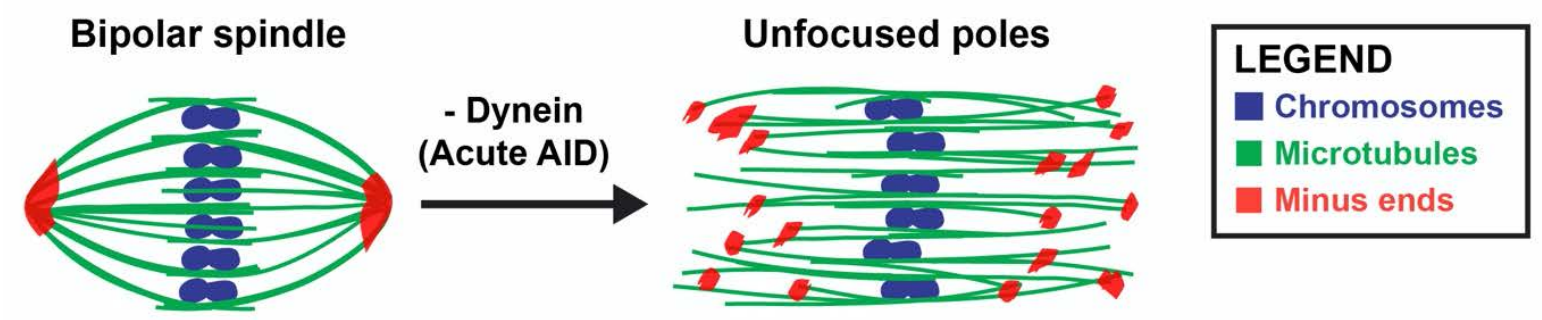

\section{Monopolar spindle (KLP-18 depletion)}

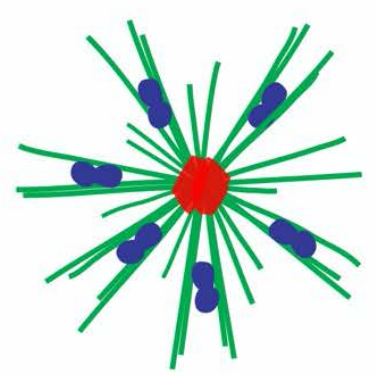

\section{Monopole breakdown}

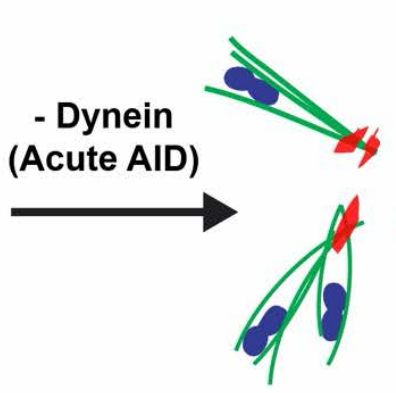

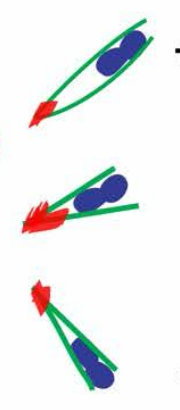

+ Kinesin-5/ BMK-1

\section{MT reorganization,} chromosome segregation

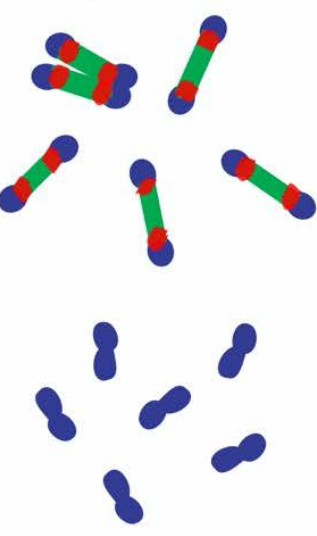

No segregation Figure 7. Dynein, KLP-18, and BMK-1 work in concert to establish and maintain spindle bipolarity in C. elegans oocyte meiosis.

796 Chromosomes (blue), microtubules (green), and microtubule minus ends (red). Dynein is

797 required throughout the meiotic divisions to maintain focused acentrosomal poles. If removed

798 from stable bipolar spindles (top), poles rapidly unfocus and splay, leading to the same

799 phenotype as long-term depletions. Dynein depletion from monopolar spindles (bottom) ejects

800 individual chromosomes and associated microtubule bundles into the cytoplasm. BMK-1 is able

801 to provide an outward sorting force in the absence of KLP-18 and dynein, enabling

802 reorganization of microtubules into a miniature anaphase spindle, promoting chromosome

803 segregation. In the absence of BMK-1, these miniature anaphases cannot form and anaphase-

804 like segregations no longer occur. 


\section{SUPPLEMENTAL VIDEOS}

\section{Video 1. Localization of dynein during oocyte meiosis.}

Shows an embryo expressing mCherry::tubulin, dissected into a control Meiosis Media solution. DHC-1::degron::GFP localization can be seen at spindle poles during metaphase, alongside faint localization to kinetochores. Localization is consistent across all embryos filmed $(n=2)$. Time elapsed shown in $(\mathrm{min}):(\mathrm{sec})$. Scale bar $=5 \mu \mathrm{m}$.

\section{Video 2. Dynein localizes to spindle poles in metaphase-arrested spindles.}

Shows a metaphase-arrested embryo expressing mCherry::tubulin (top), dissected into a control Meiosis Media solution. DHC-1::degron::GFP (bottom) is clearly localized to spindle poles, and faintly seen at kinetochores. Corresponds to Figure 2C. Localization is consistent across all embryos filmed $(n=3)$. Time elapsed shown in $(\min ):(\mathrm{sec})$. Scale bar $=5 \mu \mathrm{m}$.

\section{Video 3. Auxin treatment rapidly depletes dynein and unfocuses poles.}

Shows a metaphase-arrested embryo expressing mCherry::tubulin (top), dissected into Meiosis Media containing $100 \mu \mathrm{M}$ auxin to deplete dynein. Once dissected into auxin solution, rapid depletion of DHC-1::degron::GFP (bottom) is evident within 3 minutes, at which point spindle poles begin to unfocus and splay apart. Corresponds to Figure 2C. Phenotypes are consistent across all embryos filmed $(n=4)$. Time elapsed shown in (min):(sec). Scale bar $=5 \mu \mathrm{m}$.

\section{Video 4. Metaphase-arrested spindles remain stable and maintain chromosome} alignment.

Shows a metaphase-arrested embryo expressing GFP::tubulin and GFP::histone, dissected into a control Meiosis Media solution. No major changes in spindle length or shape occur and chromosomes are stably aligned in the spindle center. Corresponds to Figure 2D. Phenotypes are consistent across all embryos filmed $(n=5)$. Time elapsed shown in $(\min ):(\sec )$. Scale bar $=$ $5 \mu \mathrm{m}$.

\section{Video 5. Auxin treatment unfocuses poles, but spindle and chromosomes largely retain} alignment along a single axis.

Shows a metaphase-arrested embryo expressing GFP::tubulin and GFP::histone, dissected into Meiosis Media containing 100 $\mathrm{M}$ auxin to deplete dynein. Upon dissection into auxin solution, rapid and dynamic splaying of acentrosomal poles occurs alongside a notable increase in spindle length, but microtubule bundles and chromosomes stay somewhat aligned along a 
single axis. Corresponds to Figure 2D. Phenotypes are consistent across all embryos filmed $(n=6)$. Time elapsed shown in $(\min )$ :(sec). Scale bar $=5 \mu \mathrm{m}$.

\section{Video 6. Normal embryos undergo two rounds of bipolar spindle formation and anaphase} segregation.

Shows an unarrested embryo expressing GFP::tubulin and GFP::histone, dissected into a control Meiosis Media solution. After one round of segregation, a second bipolar spindle is formed, rotates perpendicular to the cortex, and successfully extrudes a second polar body. Corresponds to Figure 2 - figure supplement 2. Phenotypes are consistent across all embryos filmed $(n=5)$. Time elapsed shown in $(\min ):(\mathrm{sec})$. Scale bar $=5 \mu \mathrm{m}$.

\section{Video 7. Splaying of acentrosomal poles occurs in unarrested embryos.}

Shows an unarrested embryo expressing GFP::tubulin and GFP::histone, dissected into Meiosis Media containing $100 \mu \mathrm{M}$ auxin to deplete dynein. A spindle tries to form in the absence of dynein, leading to dynamic and unfocused poles. Anaphase segregation still occurs, albeit with a failure in spindle rotation. Corresponds to Figure 2 - figure supplement 2. Phenotypes are consistent across all embryos filmed $(n=7)$. Time elapsed shown in $(\min ):(\mathrm{sec})$. Scale bar $=$ $5 \mu \mathrm{m}$.

\section{Video 8. Complete dissolution of monopoles occurs upon auxin addition.}

Shows an embryo expressing GFP::tubulin and GFP::histone following klp-18(RNAi), dissected into Meiosis Media containing $100 \mu \mathrm{M}$ auxin to deplete dynein. Upon dissection into auxin solution, the monopole quickly dissolves, leading to ejection of individual chromosomes (with laterally-associated microtubule bundles) into the cytoplasm, leading to an anaphase-like segregation event. Corresponds to Figure 4A. Phenotypes are consistent across all embryos filmed $(n=5)$. Time elapsed shown in $(\min ):(\mathrm{sec})$. Scale bar $=5 \mu \mathrm{m}$.

\section{Video 9. Dynamics of normal monopolar spindle.}

Shows an embryo expressing GFP::tubulin and GFP::histone following klp-18(RNAi), dissected into a Meiosis Media control solution. Chromosomes extend outward towards microtubule plus ends forming a monopolar spindle during metaphase, but retract towards the minus ends during anaphase. Corresponds to Figure 4A. Phenotypes are consistent across all embryos filmed $(n=6)$. Time elapsed shown in $(\mathrm{min})$ :(sec). Scale bar $=5 \mu \mathrm{m}$.

\section{Video 10. Microtubule reorganization leads to anaphase-like segregation for individual} chromosomes. 
875 Shows an embryo expressing GFP::tubulin and GFP::histone following klp-18(RNAi), dissected

876 into Meiosis Media containing $100 \mu \mathrm{M}$ auxin to deplete dynein. After breakdown of the

877 monopolar spindle, reorganization of local microtubule bundles around individual chromosomes

878 can be observed. Shortly after reorganization of these miniature spindles, an anaphase-like

879 segregation occurs synchronously across all chromosomes. Corresponds to Figure 4B.

880 Phenotypes are consistent across all embryos filmed ( $n=5)$. Time elapsed shown in (min):(sec).

881 Scale bar $=5 \mu \mathrm{m}$.

882

883 Video 11. Monopolar spindle dynamics are normal in the absence of BMK-1.

884 Shows a bmk-1(syb3914) embryo expressing GFP::tubulin and GFP::histone following klp-

885 18(RNAi) dissected into a control Meiosis Media solution. Without functional BMK-1 or KLP-18,

886 monopolar spindles can still form and monopolar anaphase still occurs. Phenotypes are

887 consistent across all embryos filmed $(n=4)$. Time elapsed shown in (min):(sec). Scale bar =

$8885 \mu \mathrm{m}$.

889

890 Video 12. Loss of BMK-1 function prevents the formation of miniature anaphases.

891 Shows a bmk-1(syb3914) embryo expressing GFP::tubulin and GFP::histone following klp-

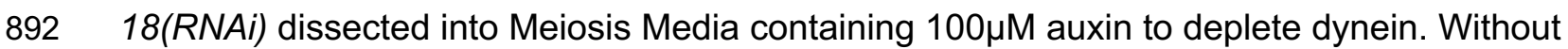

893 functional BMK-1, formation of miniature bipolar anaphases do not occur after the breakdown of

894 the monopolar spindle. Corresponds to Figure 6D. Phenotypes are consistent across all

895 embryos filmed $(n=3)$. Time elapsed shown in $(\min ):(\mathrm{sec})$. Scale bar $=5 \mu \mathrm{m}$. 


\section{REFERENCES}

Albertson, D.G., and J.N. Thomson. 1993. Segregation of holocentric chromosomes at meiosis in the nematode, Caenorhabditis elegans. Chromosome Res. 1:15-26.

Bishop, J.D., Z. Han, and J.M. Schumacher. 2005. The Caenorhabditis elegans Aurora B kinase AIR-2 phosphorylates and is required for the localization of a BimC kinesin to meiotic and mitotic spindles. Mol Biol Cell. 16:742-756.

Borgal, L., and J.G. Wakefield. 2018. Context-dependent spindle pole focusing. Essays Biochem. 62:803-813.

Connolly, A.A., V. Osterberg, S. Christensen, M. Price, C. Lu, K. Chicas-Cruz, S. Lockery, P.E. Mains, and B. Bowerman. 2014. Caenorhabditis elegans oocyte meiotic spindle pole assembly requires microtubule severing and the calponin homology domain protein ASPM-1. Mol Biol Cell. 25:1298-1311.

Connolly, A.A., K. Sugioka, C.H. Chuang, J.B. Lowry, and B. Bowerman. 2015. KLP-7 acts through the Ndc80 complex to limit pole number in $\mathrm{C}$. elegans oocyte meiotic spindle assembly. J Cell Biol. 210:917-932.

Crowder, M.E., J.R. Flynn, K.P. McNally, D.B. Cortes, K.L. Price, P.A. Kuehnert, M.T. Panzica, A. Andaya, J.A. Leary, and F.J. McNally. 2015. Dynactin-dependent cortical dynein and spherical spindle shape correlate temporally with meiotic spindle rotation in Caenorhabditis elegans. Mol Biol Cell. 26:3030-3046.

Danlasky, B.M., M.T. Panzica, K.P. McNally, E. Vargas, C. Bailey, W. Li, T. Gong, E.S. Fishman, X. Jiang, and F.J. McNally. 2020. Evidence for anaphase pulling forces during C. elegans meiosis. J Cell Biol. 219.

Davis-Roca, A.C., N.S. Divekar, R.K. Ng, and S.M. Wignall. 2018. Dynamic SUMO remodeling drives a series of critical events during the meiotic divisions in Caenorhabditis elegans. PLoS Genet. 14:e1007626.

Davis-Roca, A.C., C.C. Muscat, and S.M. Wignall. 2017. Caenorhabditis elegans oocytes detect meiotic errors in the absence of canonical end-on kinetochore attachments. $J$ Cell Biol. 216:1243-1253.

Divekar, N.S., A.C. Davis-Roca, L. Zhang, A.F. Dernburg, and S.M. Wignall. 2021a. A degronbased strategy reveals new insights into Aurora B function in C. elegans. PLoS Genet. $17: \mathrm{e} 1009567$.

Divekar, N.S., H.E. Horton, and S.M. Wignall. 2021b. Methods for Rapid Protein Depletion in C. elegans using Auxin-Inducible Degradation. Curr Protoc. 1:e16.

Dumont, J., K. Oegema, and A. Desai. 2010. A kinetochore-independent mechanism drives anaphase chromosome separation during acentrosomal meiosis. Nature cell biology. 12:894-901.

Ellefson, M.L., and F.J. McNally. 2009. Kinesin-1 and cytoplasmic dynein act sequentially to move the meiotic spindle to the oocyte cortex in Caenorhabditis elegans. Mol Biol Cell. 20:2722-2730.

Ellefson, M.L., and F.J. McNally. 2011. CDK-1 inhibits meiotic spindle shortening and dyneindependent spindle rotation in C. elegans. The Journal of cell biology. 193:1229-1244. 
937

938

939

940

941

942

943

944

945

946

947

948

949

950

951

952

953

954

955

956

957

958

959

960

961

962

963

964

965

966

967

968

969

970

971

972

973

974

975

976

977

Elting, M.W., C.L. Hueschen, D.B. Udy, and S. Dumont. 2014. Force on spindle microtubule minus ends moves chromosomes. J Cell Biol. 206:245-256.

Ferenz, N.P., R. Paul, C. Fagerstrom, A. Mogilner, and P. Wadsworth. 2009. Dynein antagonizes eg5 by crosslinking and sliding antiparallel microtubules. Curr Biol. 19:18331838.

Furuta, T., S. Tuck, J. Kirchner, B. Koch, R. Auty, R. Kitagawa, A.M. Rose, and D. Greenstein. 2000. EMB-30: an APC4 homologue required for metaphase-to-anaphase transitions during meiosis and mitosis in Caenorhabditis elegans. Mol Biol Cell. 11:1401-1419.

Gigant, E., M. Stefanutti, K. Laband, A. Gluszek-Kustusz, F. Edwards, B. Lacroix, G. Maton, J.C. Canman, J.P. Welburn, and J. Dumont. 2017. Inhibition of ectopic microtubule assembly by the kinesin-13 KLP-7 prevents chromosome segregation and cytokinesis defects in oocytes. Development. 144:1674-1686.

Gonczy, P., S. Pichler, M. Kirkham, and A.A. Hyman. 1999. Cytoplasmic dynein is required for distinct aspects of MTOC positioning, including centrosome separation, in the one cell stage Caenorhabditis elegans embryo. J Cell Biol. 147:135-150.

Han, X., K. Adames, E.M. Sykes, and M. Srayko. 2015. The KLP-7 Residue S546 Is a Putative Aurora Kinase Site Required for Microtubule Regulation at the Centrosome in C. elegans. PLoS One. 10:e0132593.

Hattersley, N., D.K. Cheerambathur, M.W. Moyle, M. Stefanutti, A. Richardson, K. Lee, J. Dumont, K. Oegema, and A. Desai. 2016. A Nucleoporin Docks Protein Phosphatase 1 to Direct Meiotic Chromosome Segregation and Nuclear Assembly. Dev Cell. 38:463477.

Hollis, J.A., M.L. Glover, A.J. Schlientz, C.K. Cahoon, B. Bowerman, S.M. Wignall, and D.E. Libuda. 2020. Excess crossovers impede faithful meiotic chromosome segregation in $\mathrm{C}$. elegans. PLoS Genet. 16:e1009001.

Holubcova, Z., M. Blayney, K. Elder, and M. Schuh. 2015. Human oocytes. Error-prone chromosome-mediated spindle assembly favors chromosome segregation defects in human oocytes. Science. 348:1143-1147.

Hueschen, C.L., S.J. Kenny, K. Xu, and S. Dumont. 2017. NuMA recruits dynein activity to microtubule minus-ends at mitosis. eLife. 6.

Kamath, R.S., A.G. Fraser, Y. Dong, G. Poulin, R. Durbin, M. Gotta, A. Kanapin, N. Le Bot, S. Moreno, M. Sohrmann, D.P. Welchman, P. Zipperlen, and J. Ahringer. 2003. Systematic functional analysis of the Caenorhabditis elegans genome using RNAi. Nature. 421:231237.

Kiyomitsu, T., and I.M. Cheeseman. 2013. Cortical dynein and asymmetric membrane elongation coordinately position the spindle in anaphase. Cell. 154:391-402.

Laband, K., B. Lacroix, F. Edwards, J.C. Canman, and J. Dumont. 2018. Live imaging of C. elegans oocytes and early embryos. Methods Cell Biol. 145:217-236.

Laband, K., R. Le Borgne, F. Edwards, M. Stefanutti, J.C. Canman, J.M. Verbavatz, and J. Dumont. 2017. Chromosome segregation occurs by microtubule pushing in oocytes. Nat Commun. 8:1499. 
978

979

980

981

982

983

984

985

986

987

988

989

990

991

992

993

994

995

996

997

998

999

1000

1001

1002

1003

1004

1005

1006

1007

1008

1009

1010

1011

1012

1013

1014

1015
Mann, B.J., and P. Wadsworth. 2019. Kinesin-5 Regulation and Function in Mitosis. Trends Cell Biol. 29:66-79.

McNally, K., E. Berg, D.B. Cortes, V. Hernandez, P.E. Mains, and F.J. McNally. 2014. Katanin maintains meiotic metaphase chromosome alignment and spindle structure in vivo and has multiple effects on microtubules in vitro. Mol Biol Cell. 25:1037-1049.

McNally, K.P., M.T. Panzica, T. Kim, D.B. Cortes, and F.J. McNally. 2016. A novel chromosome segregation mechanism during female meiosis. Mol Biol Cell. 27:2576-2589.

Mitchison, T.J., P. Maddox, J. Gaetz, A. Groen, M. Shirasu, A. Desai, E.D. Salmon, and T.M. Kapoor. 2005. Roles of polymerization dynamics, opposed motors, and a tensile element in governing the length of Xenopus extract meiotic spindles. Mol Biol Cell. 16:3064-3076.

Mullen, T.J., A.C. Davis-Roca, and S.M. Wignall. 2019. Spindle assembly and chromosome dynamics during oocyte meiosis. Curr Opin Cell Biol. 60:53-59.

Mullen, T.J., and S.M. Wignall. 2017. Interplay between microtubule bundling and sorting factors ensures acentriolar spindle stability during C. elegans oocyte meiosis. PLoS Genet. 13:e1006986.

Muscat, C.C., K.M. Torre-Santiago, M.V. Tran, J.A. Powers, and S.M. Wignall. 2015. Kinetochore-independent chromosome segregation driven by lateral microtubule bundles. eLife. 4:e06462.

Nguyen-Ngoc, T., K. Afshar, and P. Gonczy. 2007. Coupling of cortical dynein and G alpha proteins mediates spindle positioning in Caenorhabditis elegans. Nat Cell Biol. 9:12941302.

Oegema, K., A. Desai, S. Rybina, M. Kirkham, and A.A. Hyman. 2001. Functional analysis of kinetochore assembly in Caenorhabditis elegans. J Cell Biol. 153:1209-1226.

Pelisch, F., L. Bel Borja, E.G. Jaffray, and R.T. Hay. 2019. Sumoylation regulates protein dynamics during meiotic chromosome segregation in C. elegans oocytes. J Cell Sci.

Pelisch, F., T. Tammsalu, B. Wang, E.G. Jaffray, A. Gartner, and R.T. Hay. 2017. A SUMODependent Protein Network Regulates Chromosome Congression during Oocyte Meiosis. Mol Cell. 65:66-77.

Raaijmakers, J.A., R.G. van Heesbeen, J.L. Meaders, E.F. Geers, B. Fernandez-Garcia, R.H. Medema, and M.E. Tanenbaum. 2012. Nuclear envelope-associated dynein drives prophase centrosome separation and enables Eg5-independent bipolar spindle formation. Embo J. 31:4179-4190.

Saunders, A.M., J. Powers, S. Strome, and W.M. Saxton. 2007. Kinesin-5 acts as a brake in anaphase spindle elongation. Curr Biol. 17:R453-454.

Schuh, M., and J. Ellenberg. 2007. Self-organization of MTOCs replaces centrosome function during acentrosomal spindle assembly in live mouse oocytes. Cell. 130:484-498.

Severson, A.F., G. von Dassow, and B. Bowerman. 2016. Oocyte Meiotic Spindle Assembly and Function. Curr Top Dev Biol. 116:65-98. 
1016

1017

1018

1019

1020

1021

1022

1023

1024

1025

1026

1027

1028

1029

1030

1031

1032

1033

1034

1035

1036

1037

1038

1039

1040

1041

1042

1043

1044

1045

1046

1047

1048

1049

1050
Srayko, M., D.W. Buster, O.A. Bazirgan, F.J. McNally, and P.E. Mains. 2000. MEI-1/MEI-2 katanin-like microtubule severing activity is required for Caenorhabditis elegans meiosis. Genes Dev. 14:1072-1084.

Sturgill, E.G., S.R. Norris, Y. Guo, and R. Ohi. 2016. Kinesin-5 inhibitor resistance is driven by kinesin-12. J Cell Biol. 213:213-227.

Sturgill, E.G., and R. Ohi. 2013. Kinesin-12 differentially affects spindle assembly depending on its microtubule substrate. Curr Biol. 23:1280-1290.

Tanenbaum, M.E., L. Macurek, N. Galjart, and R.H. Medema. 2008. Dynein, Lis1 and CLIP-170 counteract Eg5-dependent centrosome separation during bipolar spindle assembly. Embo J. 27:3235-3245.

Tanenbaum, M.E., L. Macurek, A. Janssen, E.F. Geers, M. Alvarez-Fernandez, and R.H. Medema. 2009. Kif15 cooperates with eg5 to promote bipolar spindle assembly. Curr Biol. 19:1703-1711.

van der Voet, M., C.W. Berends, A. Perreault, T. Nguyen-Ngoc, P. Gonczy, M. Vidal, M. Boxem, and S. van den Heuvel. 2009. NuMA-related LIN-5, ASPM-1, calmodulin and dynein promote meiotic spindle rotation independently of cortical LIN-5/GPR/Galpha. Nature cell biology. 11:269-277.

van Heesbeen, R.G., M.E. Tanenbaum, and R.H. Medema. 2014. Balanced activity of three mitotic motors is required for bipolar spindle assembly and chromosome segregation. Cell Rep. 8:948-956.

Vanneste, D., M. Takagi, N. Imamoto, and I. Vernos. 2009. The role of Hklp2 in the stabilization and maintenance of spindle bipolarity. Curr Biol. 19:1712-1717.

Wignall, S.M., and A.M. Villeneuve. 2009. Lateral microtubule bundles promote chromosome alignment during acentrosomal oocyte meiosis. Nature cell biology. 11:839-844.

Wolff, I.D., J.A. Hollis, and S.M. Wignall. 2021. Acentrosomal spindle assembly and stability in $<$ em>C. elegans</em> oocytes requires a kinesin-12 non-motor microtubule interaction domain. bioRxiv:2021.2006.2017.448874.

Wolff, I.D., M.V. Tran, T.J. Mullen, A.M. Villeneuve, and S.M. Wignall. 2016. Assembly of C. elegans acentrosomal spindles occurs without evident MTOCs and requires microtubule sorting by KLP-18/kinesin-12 and MESP-1. Mol Biol Cell. 27:3122-3131.

Yang, H.Y., P.E. Mains, and F.J. McNally. 2005. Kinesin-1 mediates translocation of the meiotic spindle to the oocyte cortex through KCA-1, a novel cargo adapter. J Cell Biol. 169:447457.

Zhang, L., J.D. Ward, Z. Cheng, and A.F. Dernburg. 2015. The auxin-inducible degradation (AID) system enables versatile conditional protein depletion in C. elegans. Development. 
A

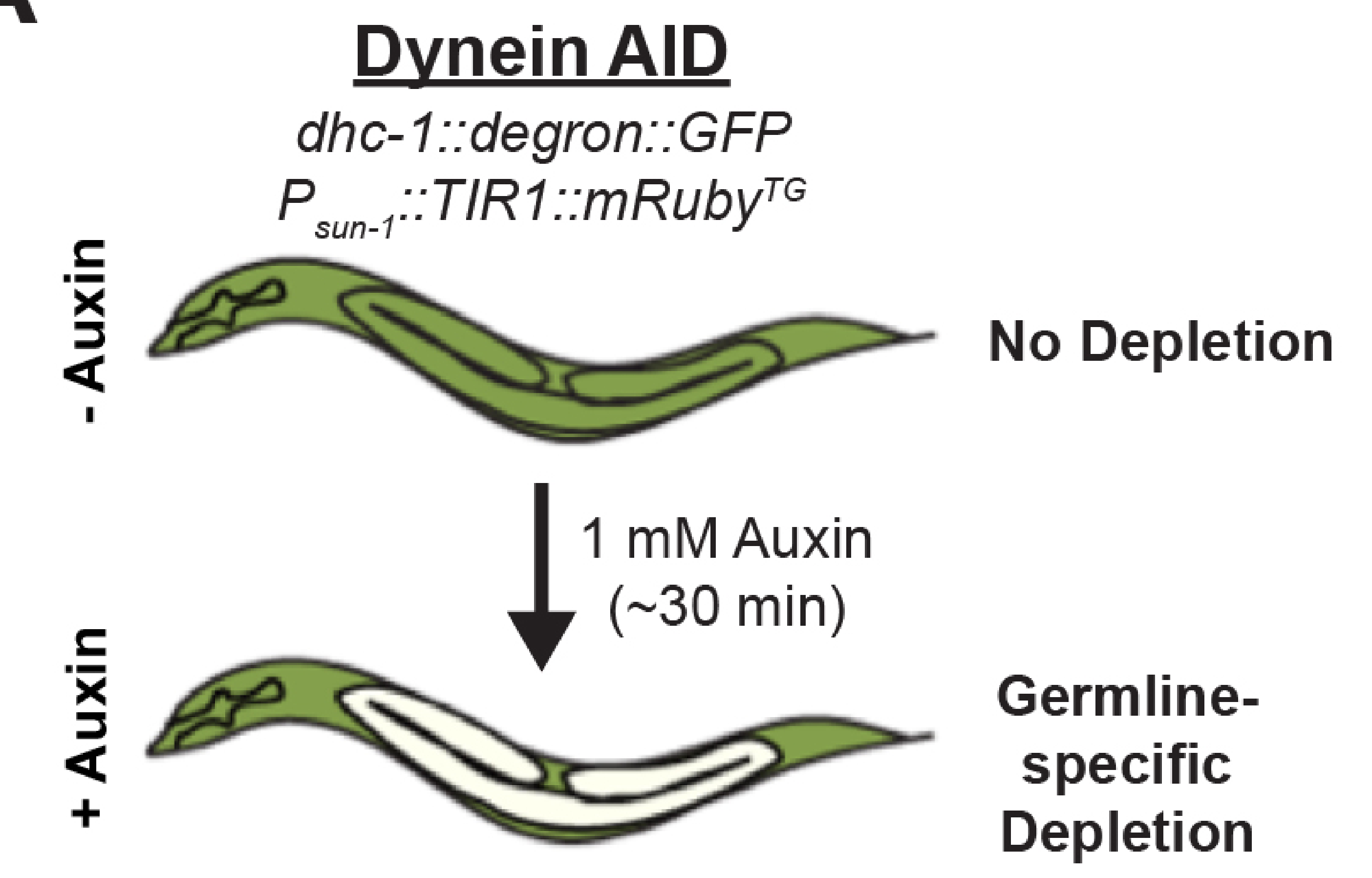

B

\section{Dynein AID strain}

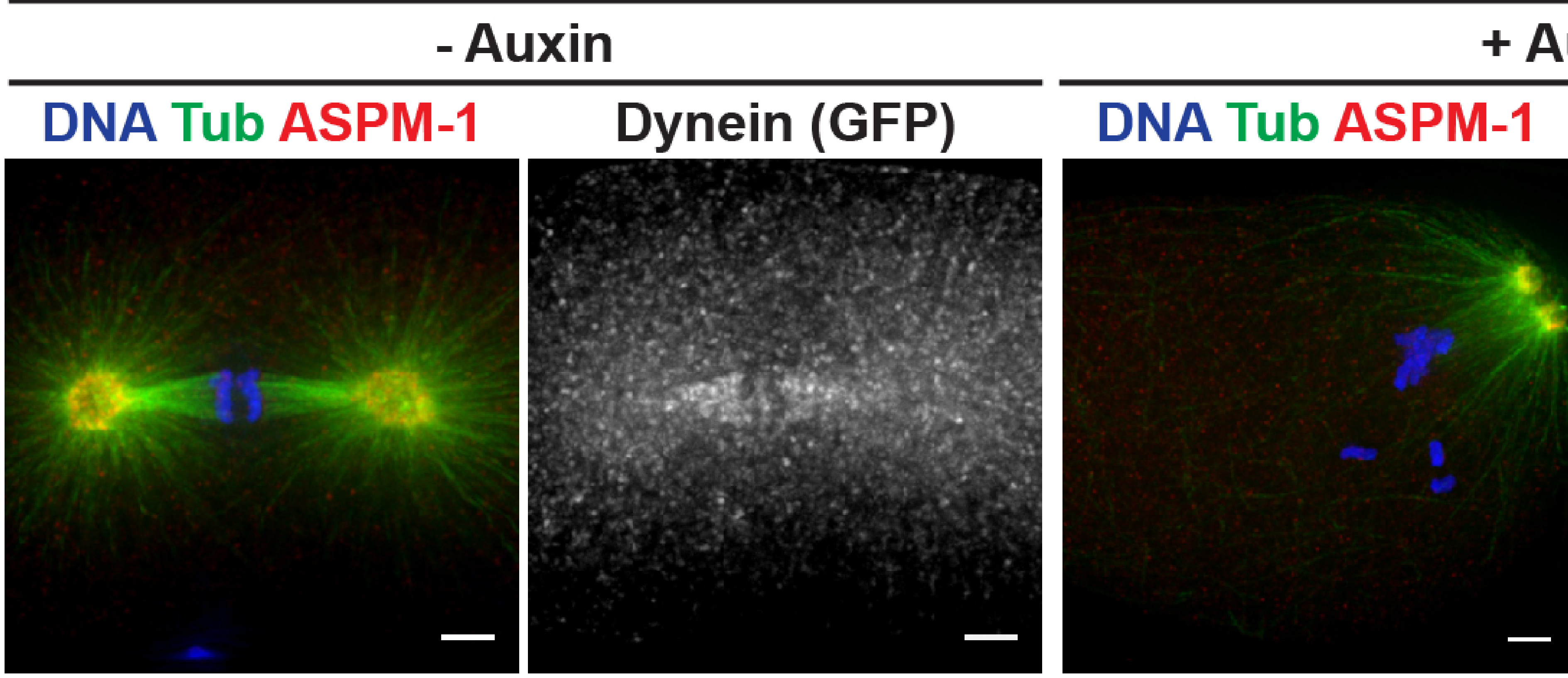

C

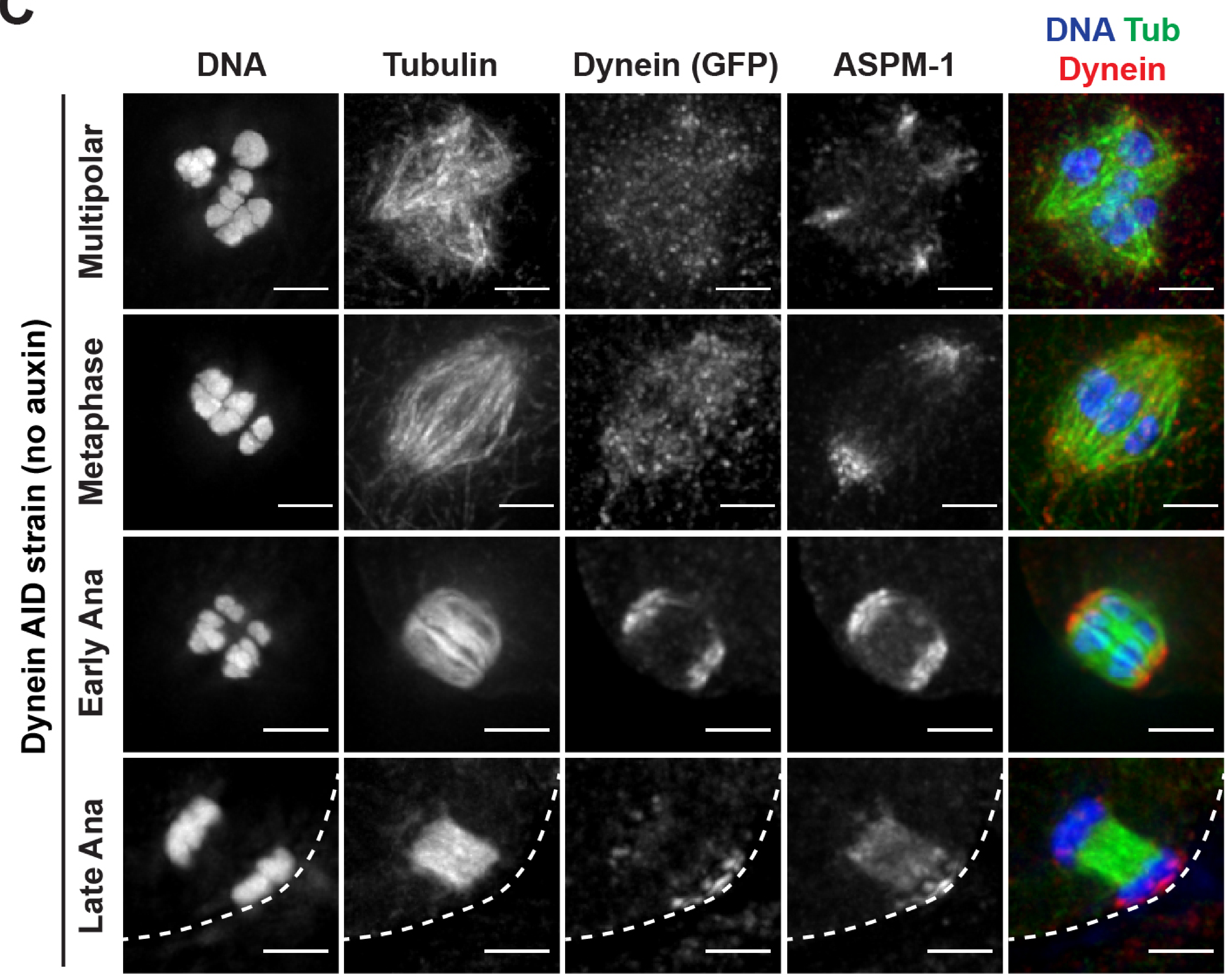

E

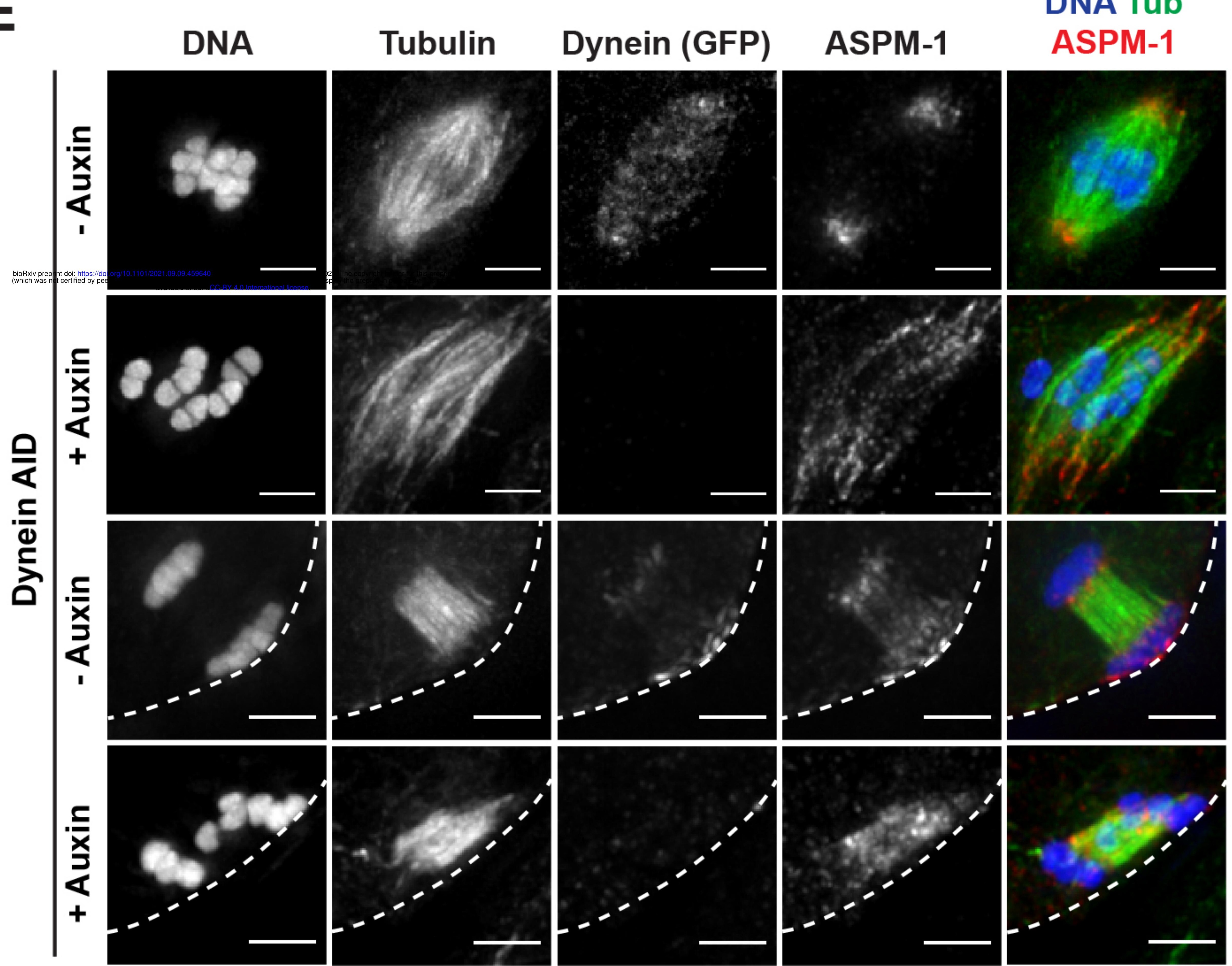

D
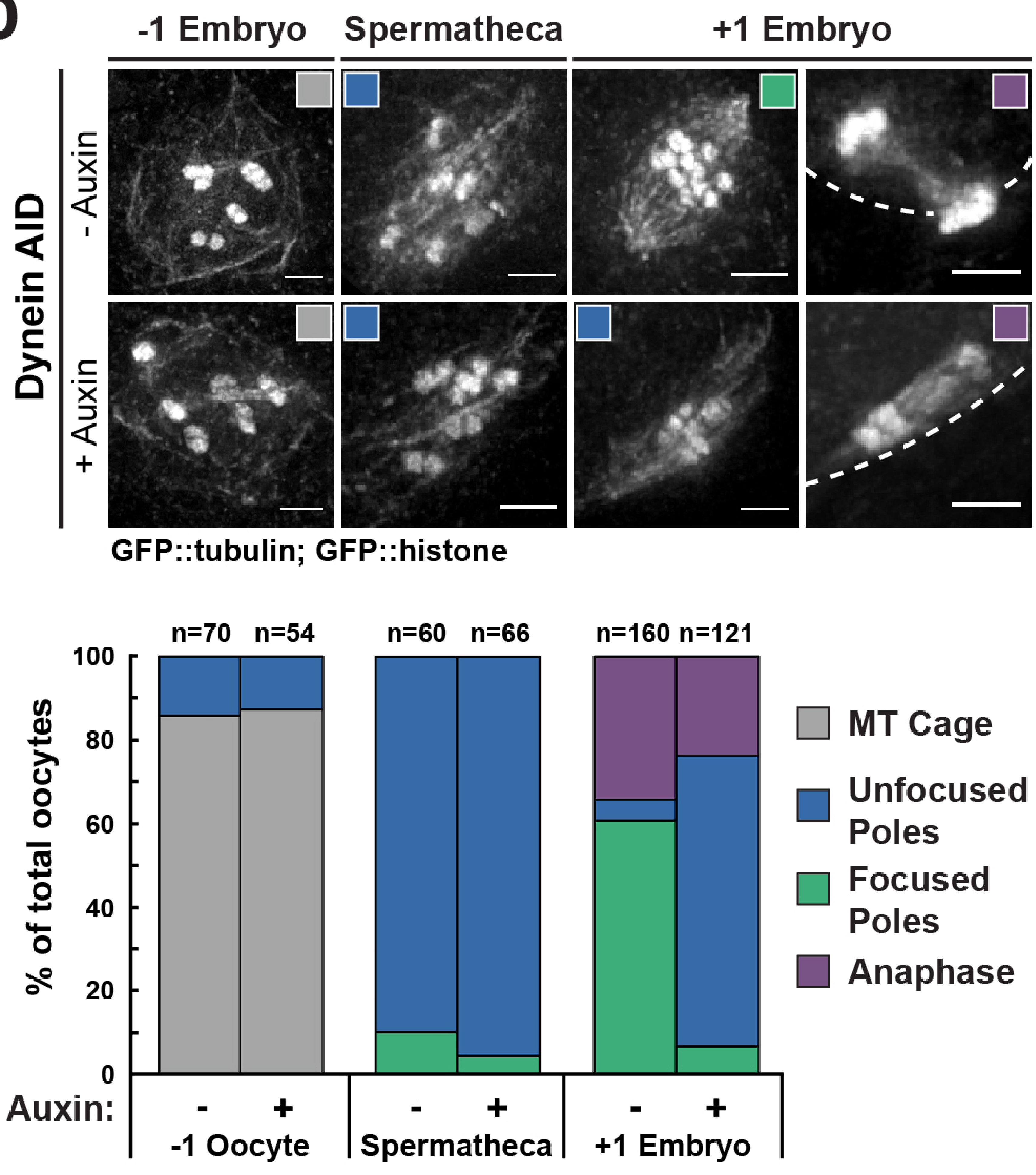

$\square$ MT Cage

Unfocused Poles

Focused Poles

Anaphase

F

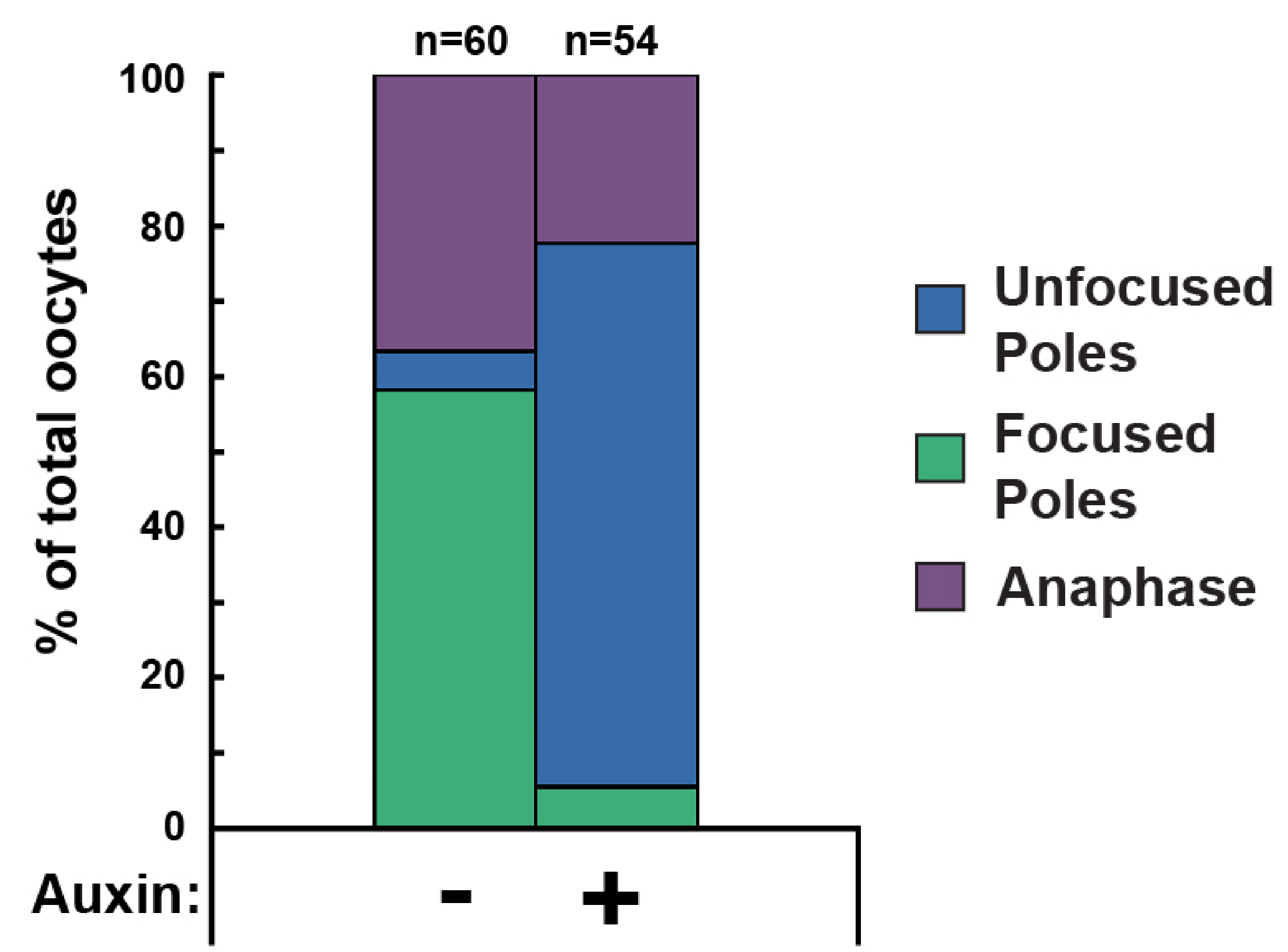


A

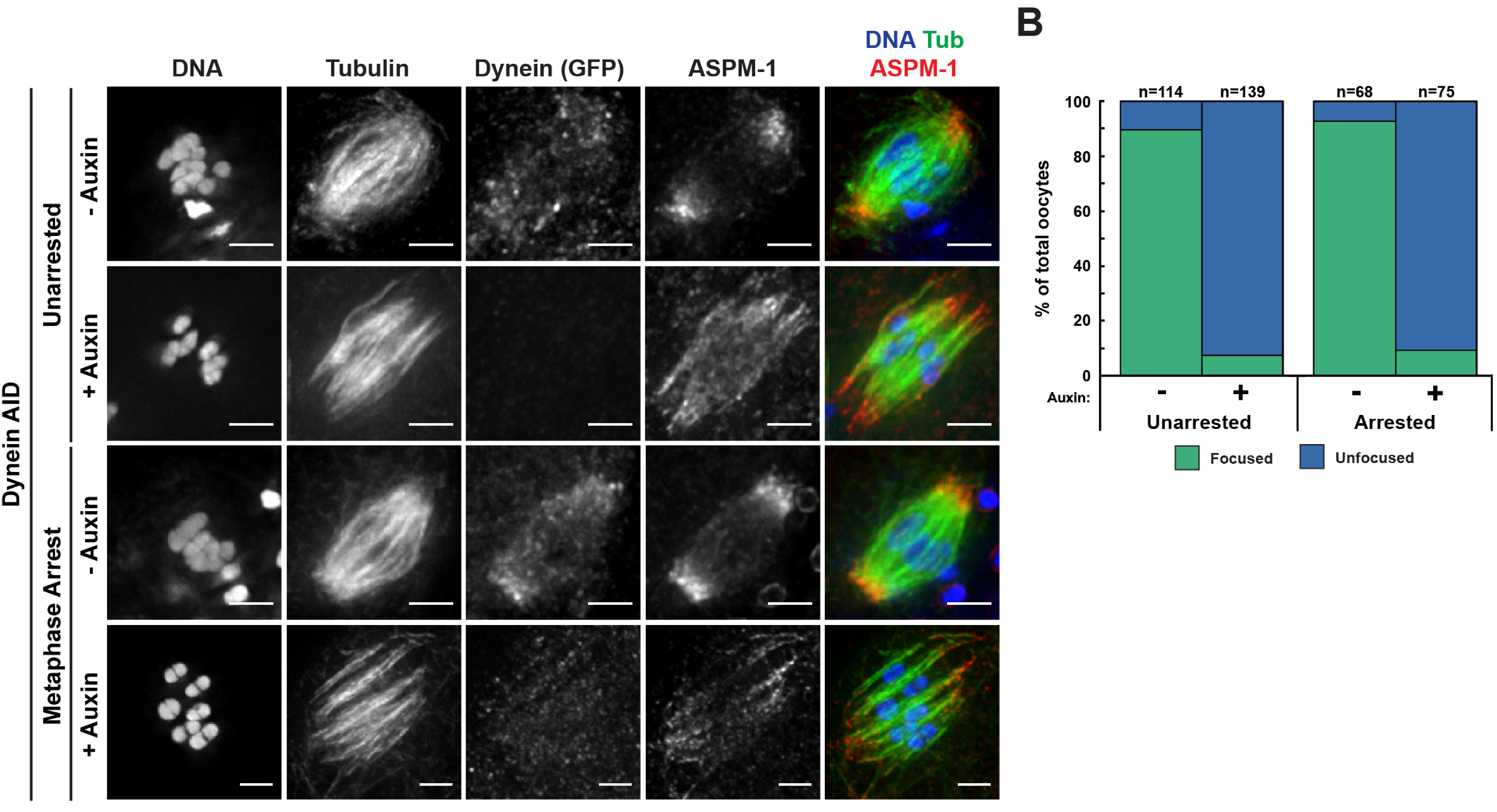

C

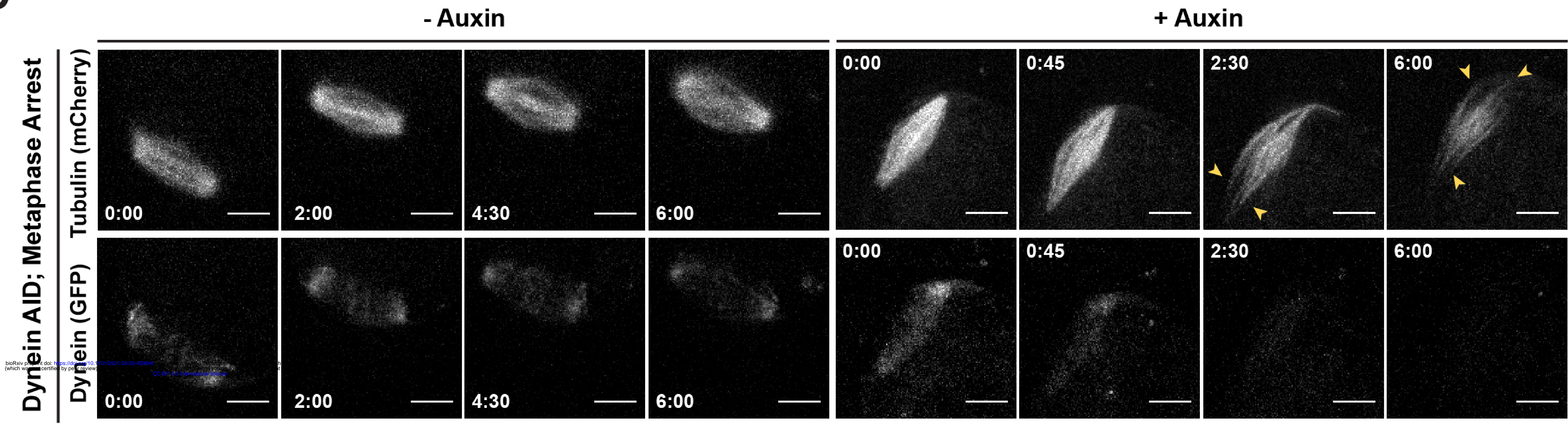

D

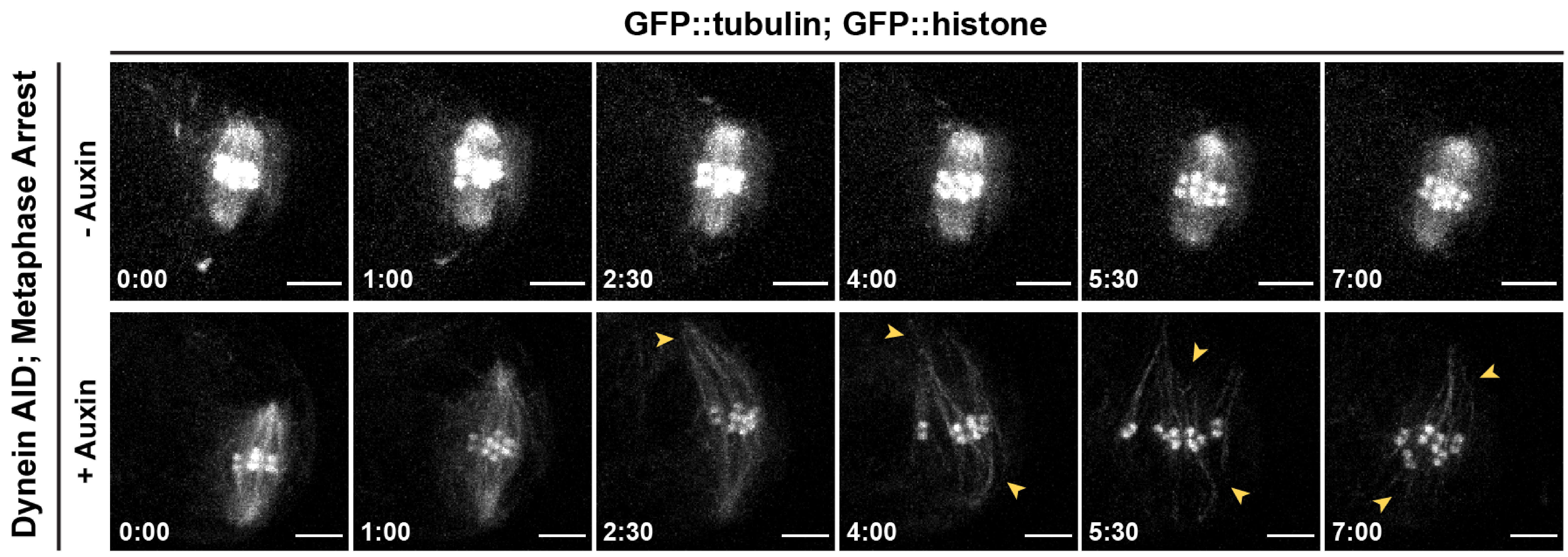




\section{FIGURE 3}

A
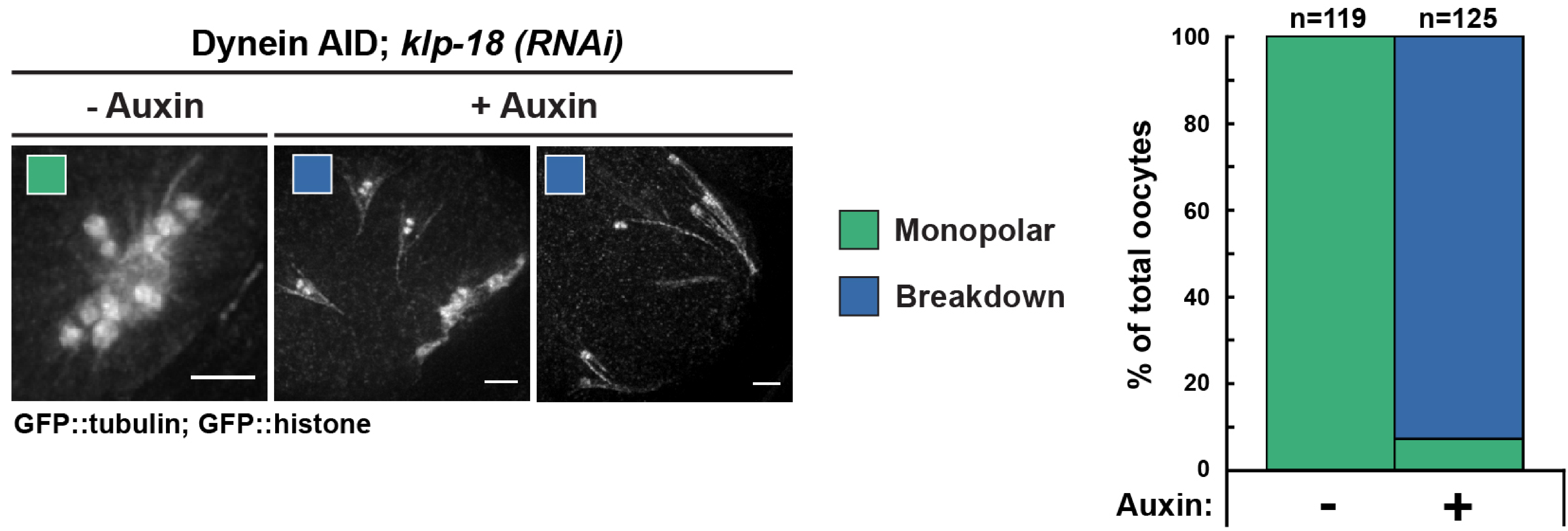

B

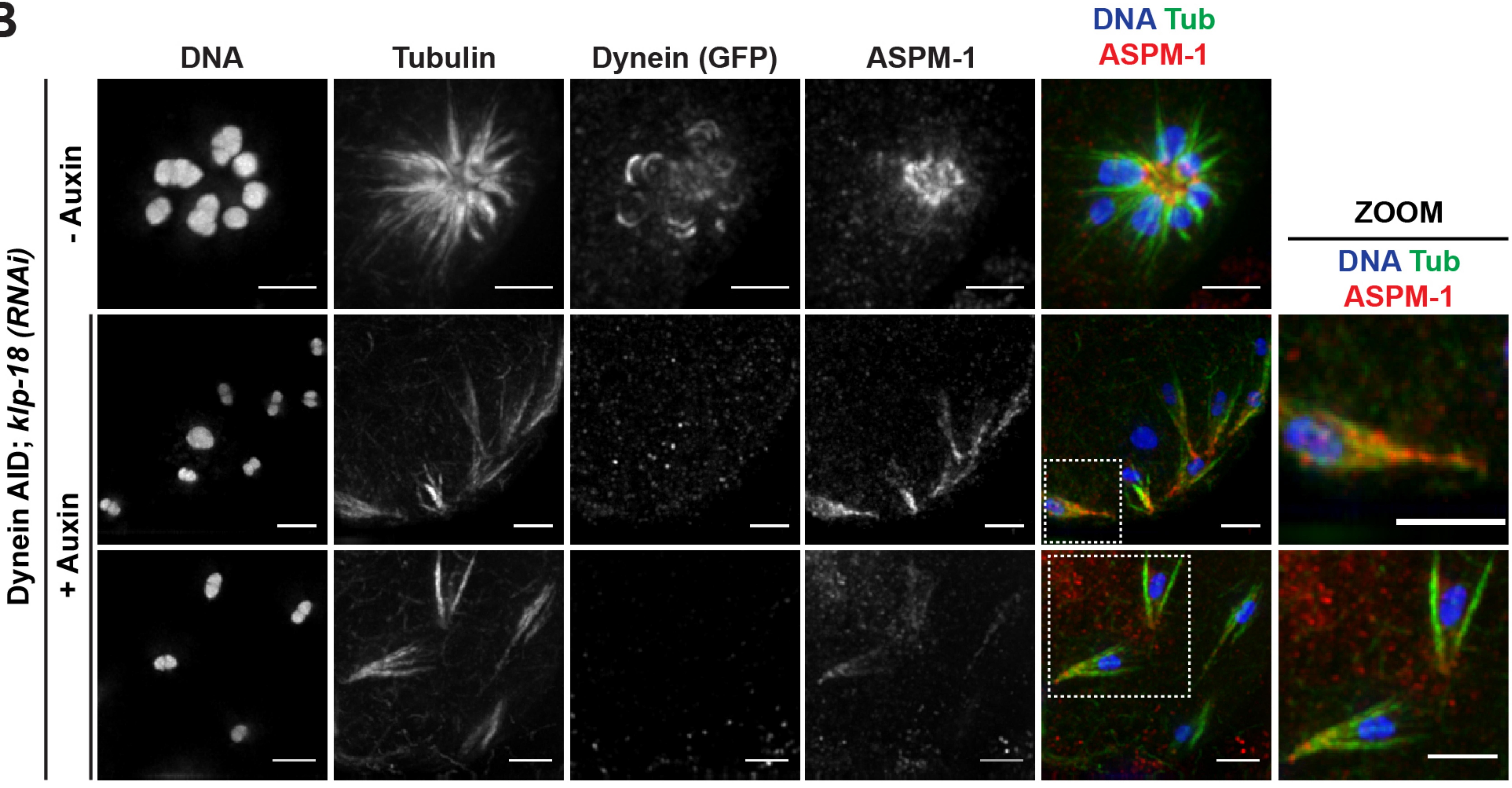

C

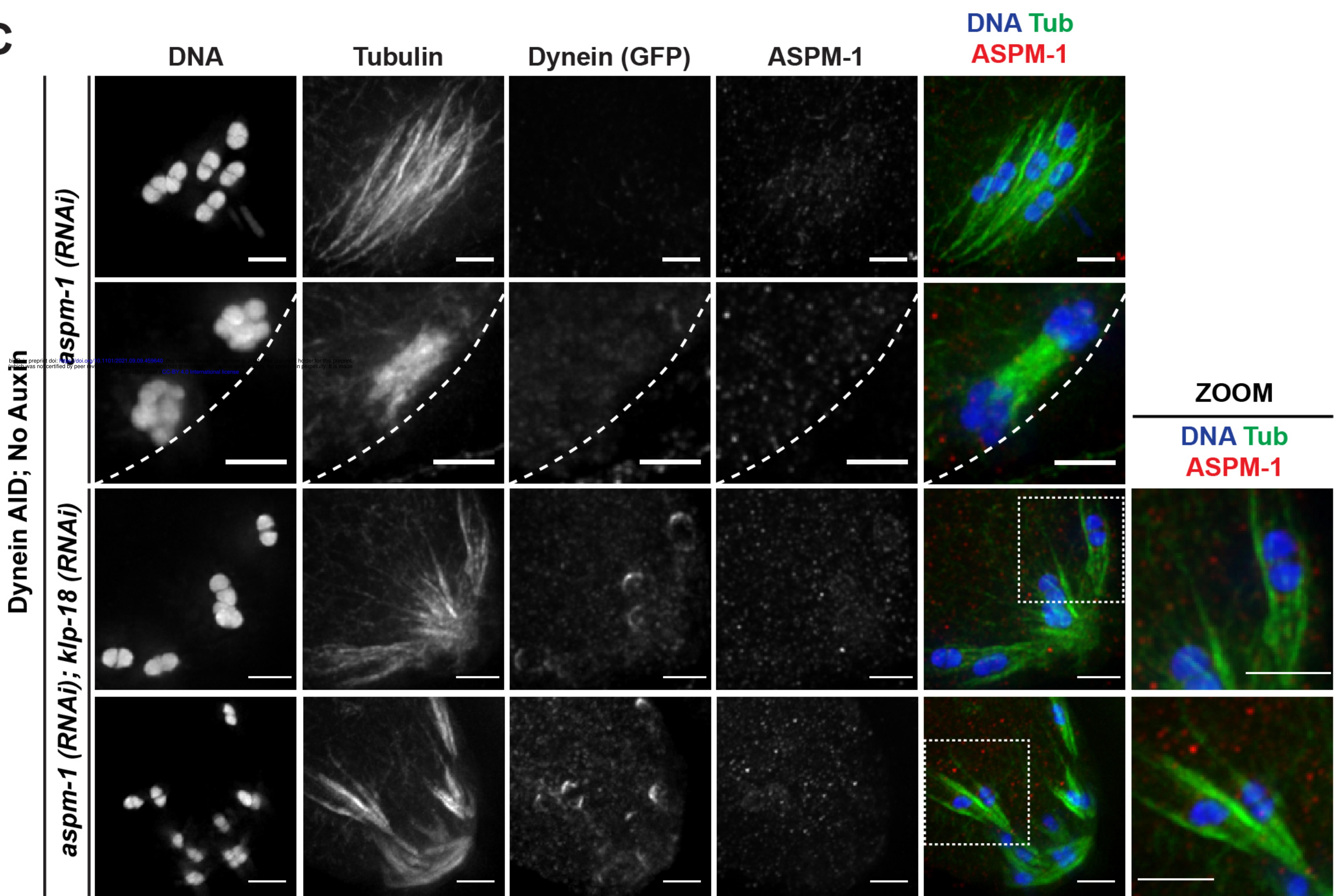


A

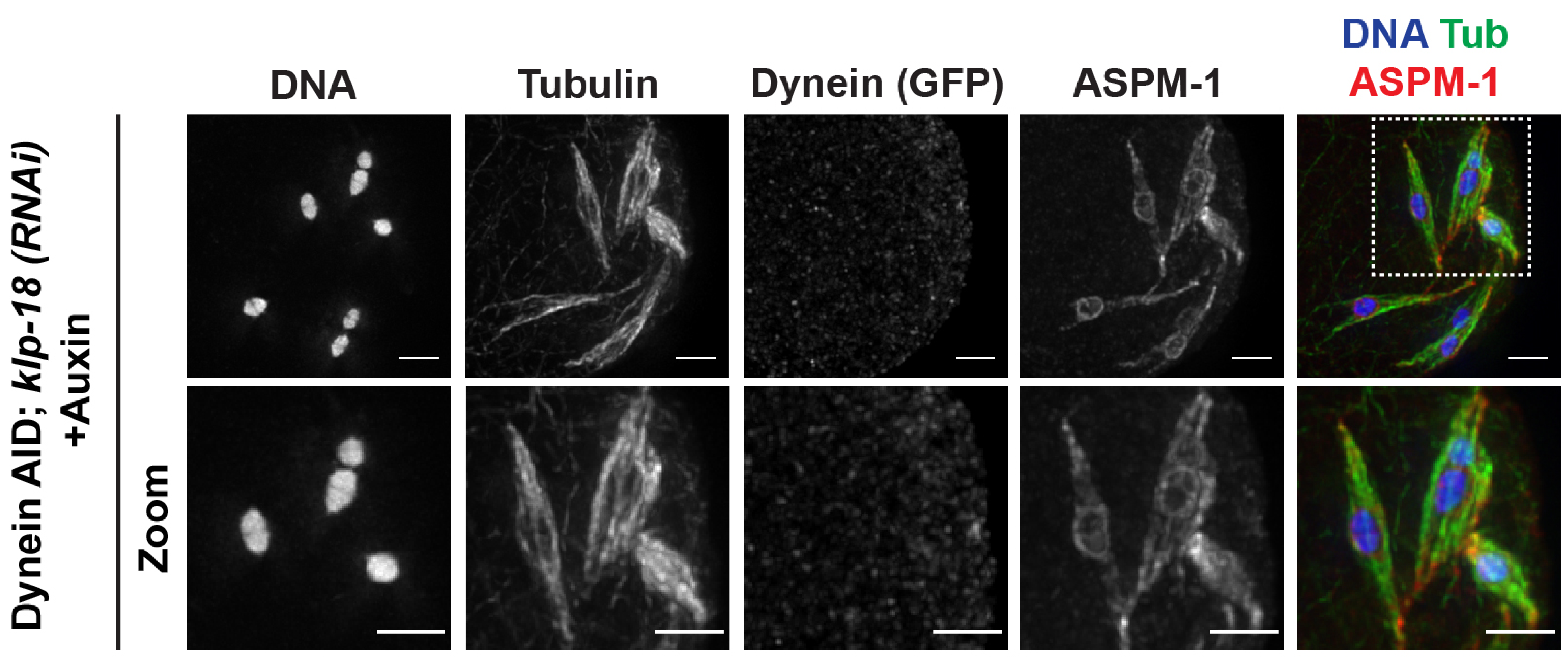

B

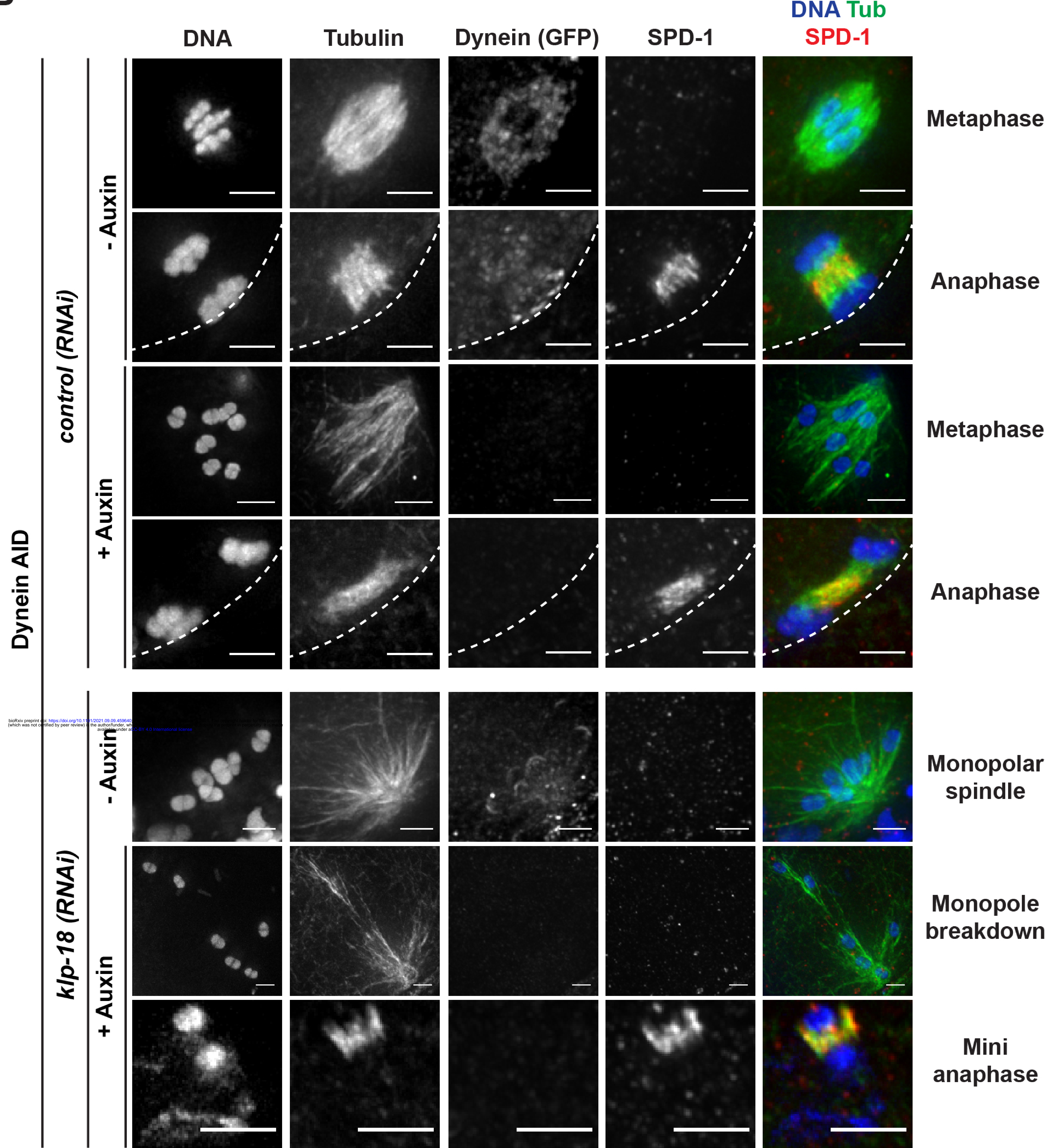


A

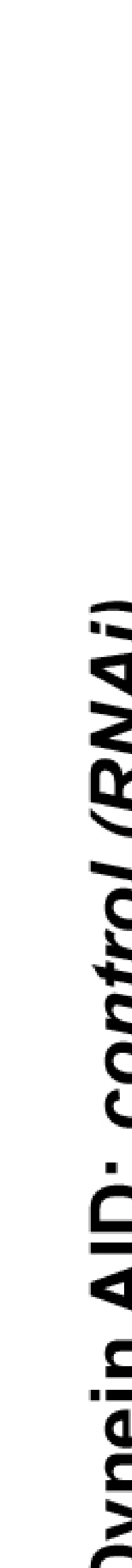



DNA

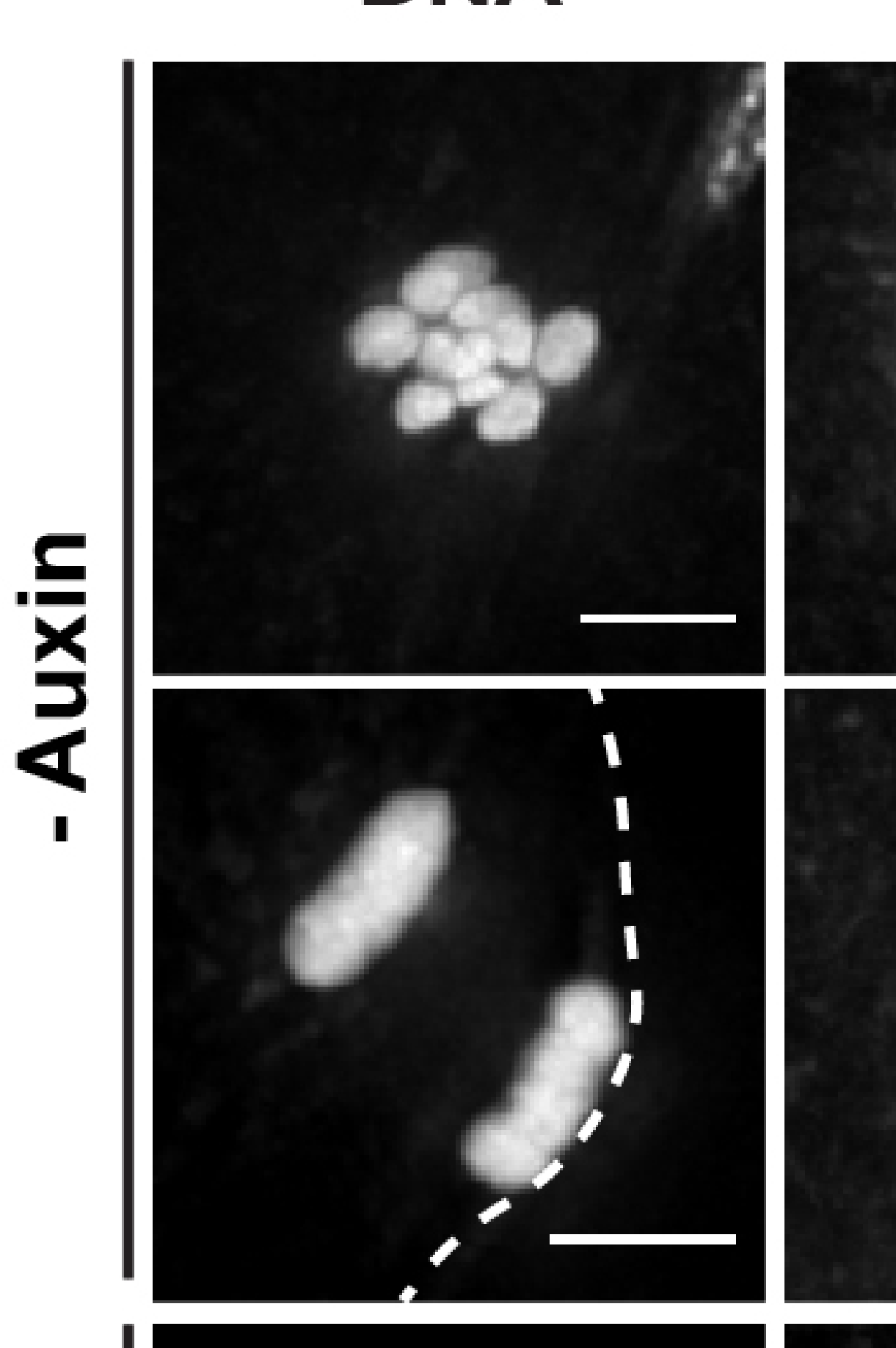

Tubulin

Dynein (GFP)

\section{BMK-1}

BMK-1

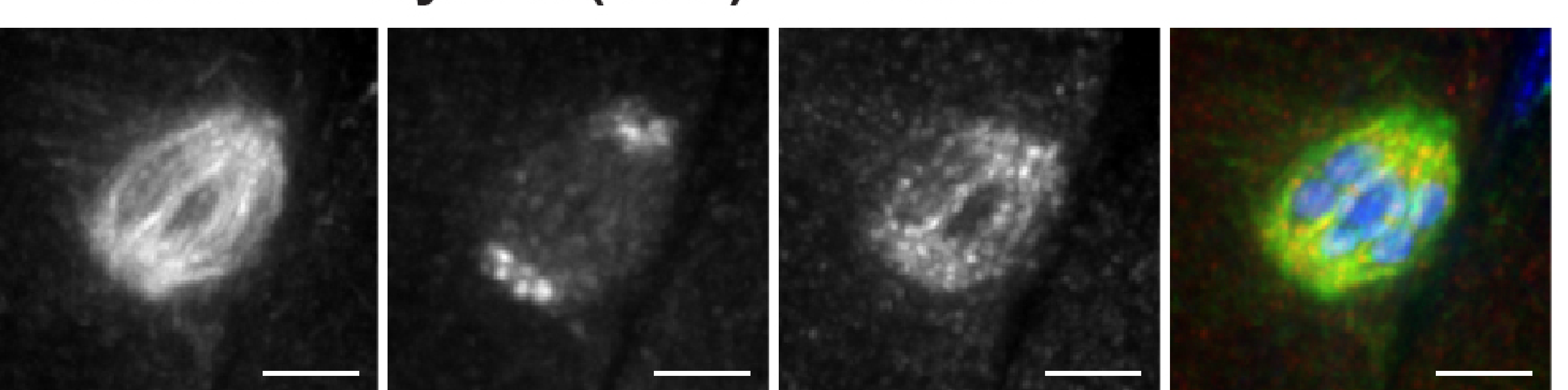

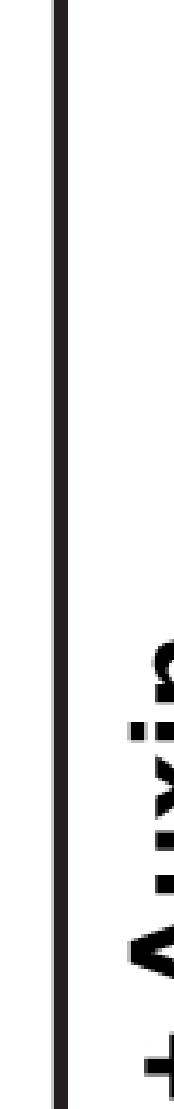
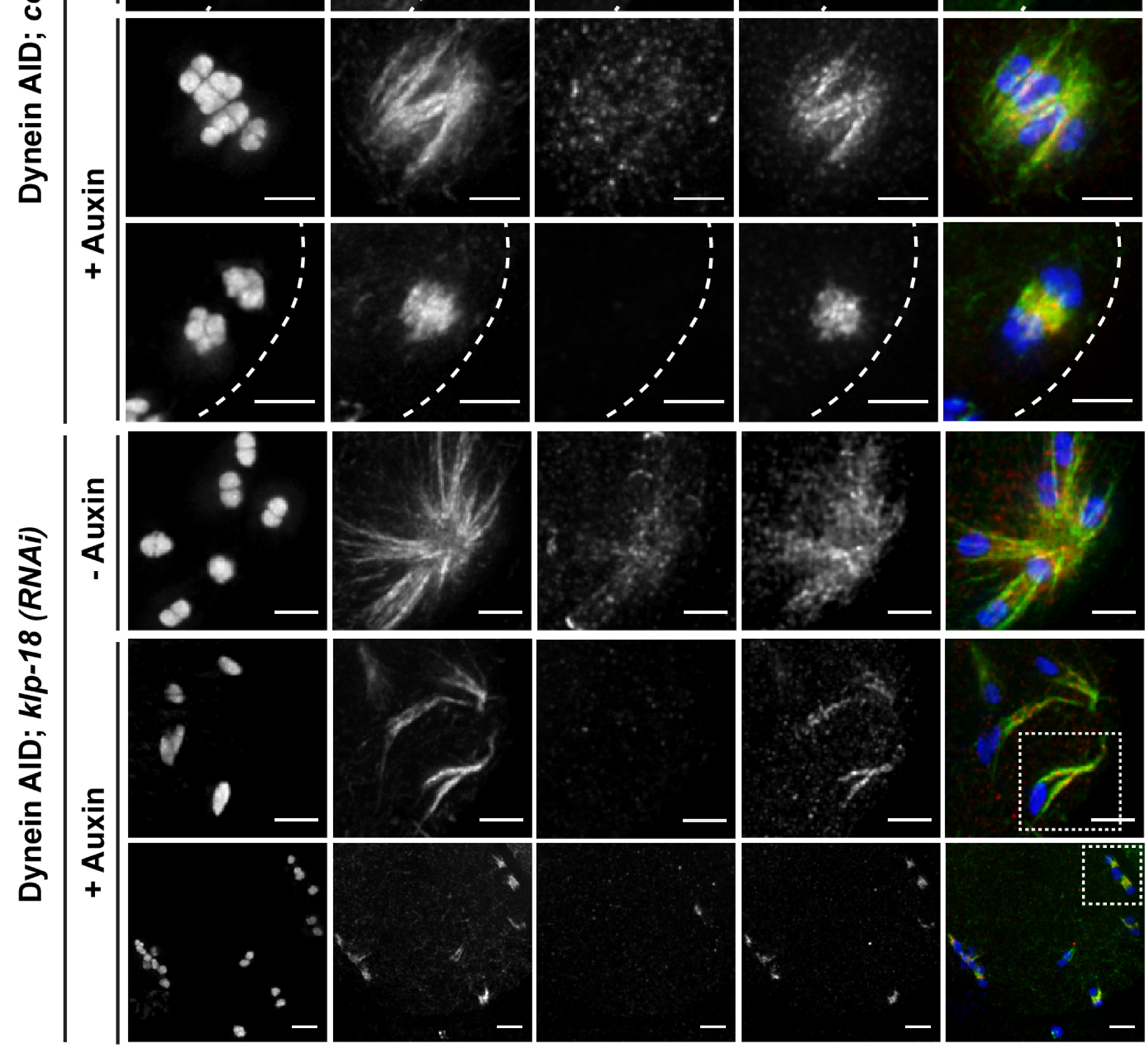

B
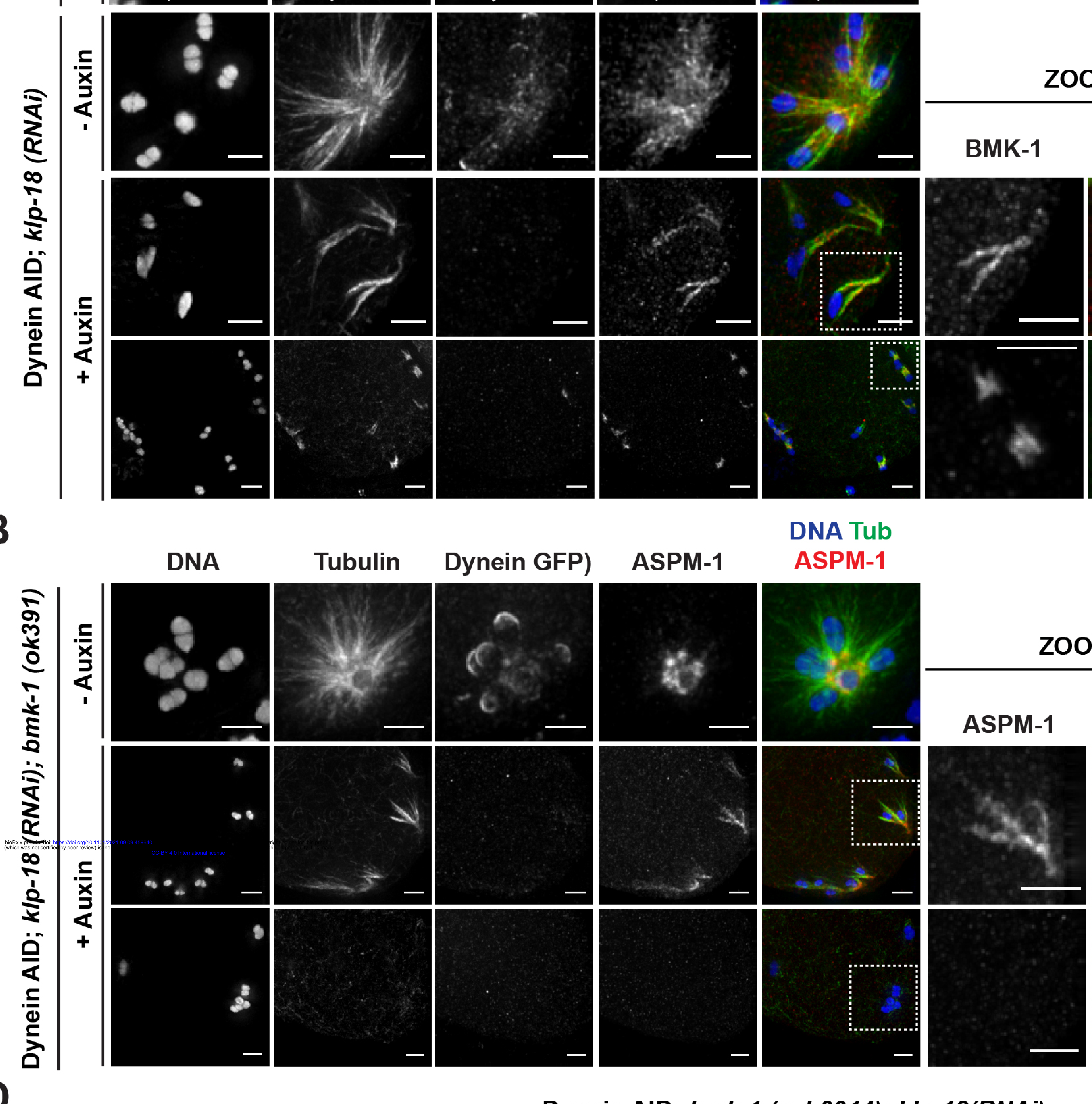

DNA Tub

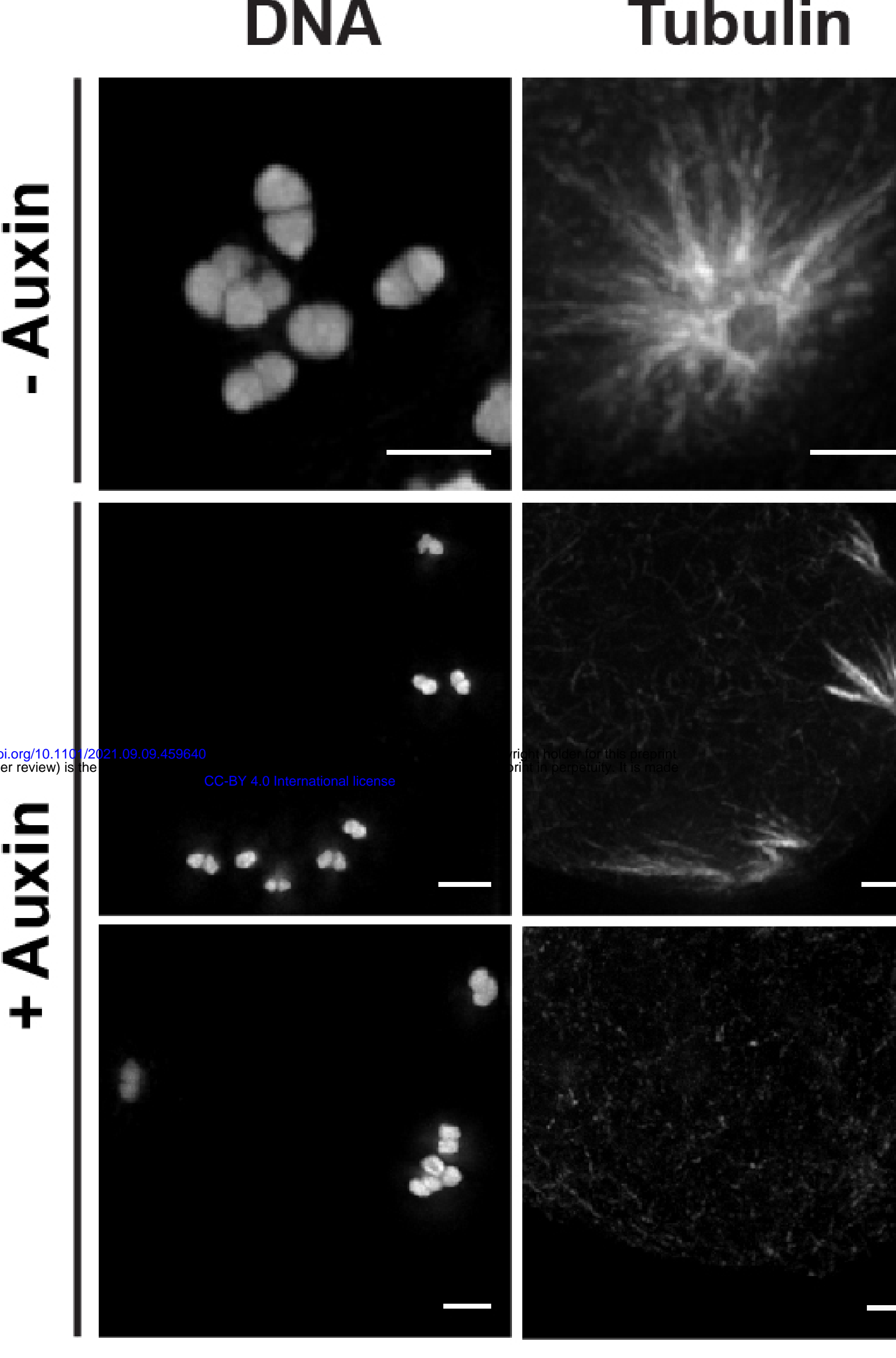

Dynein GFP) ASPM-1
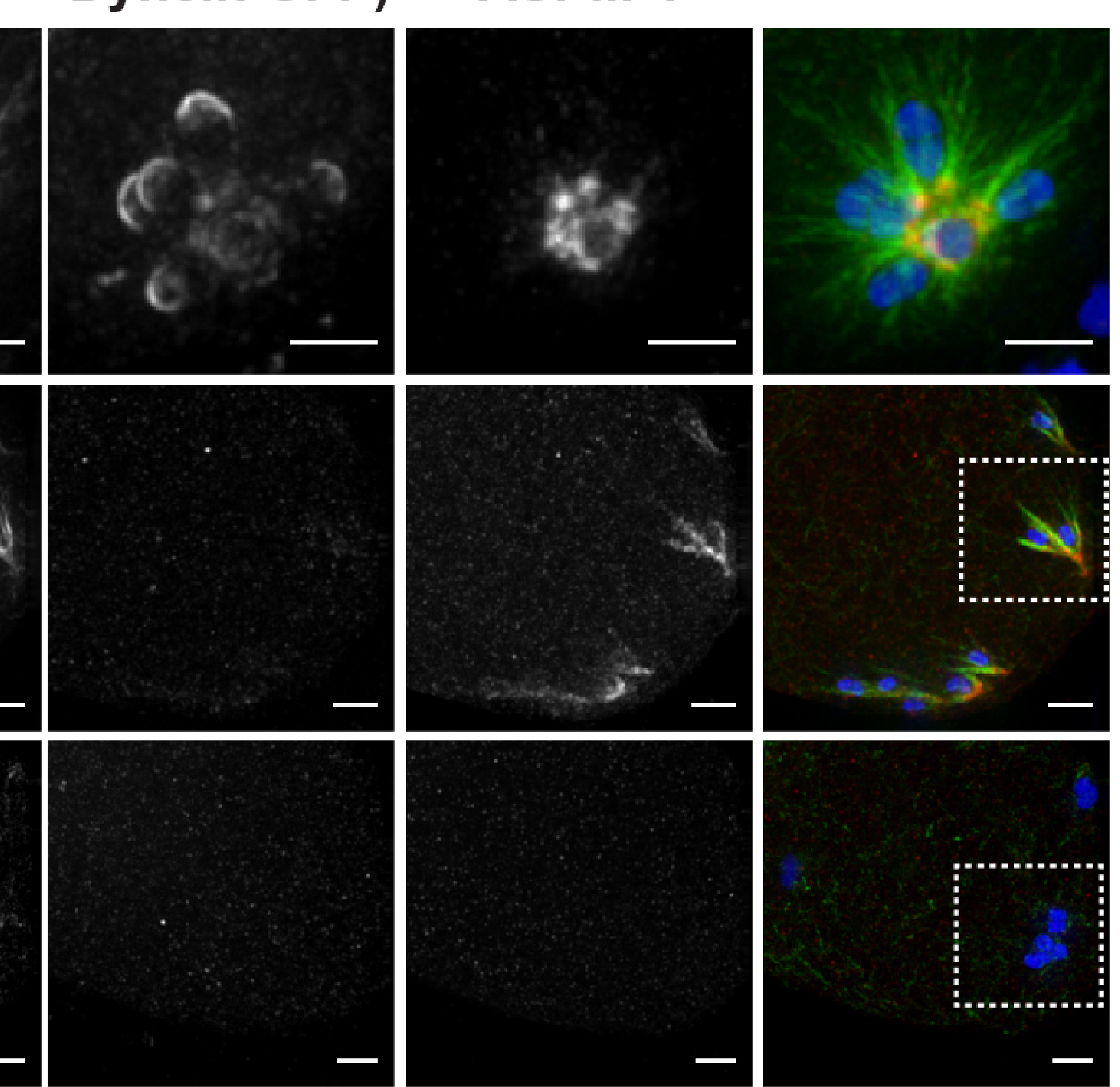

Dynein AID; bmk-1 (syb3914); klp-18(RNAi)

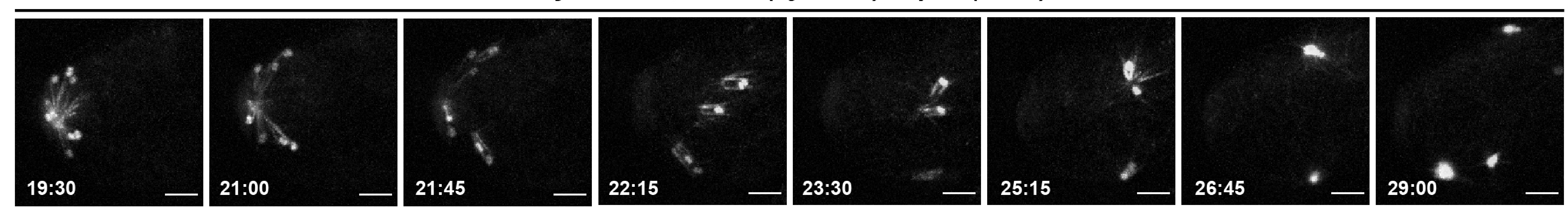

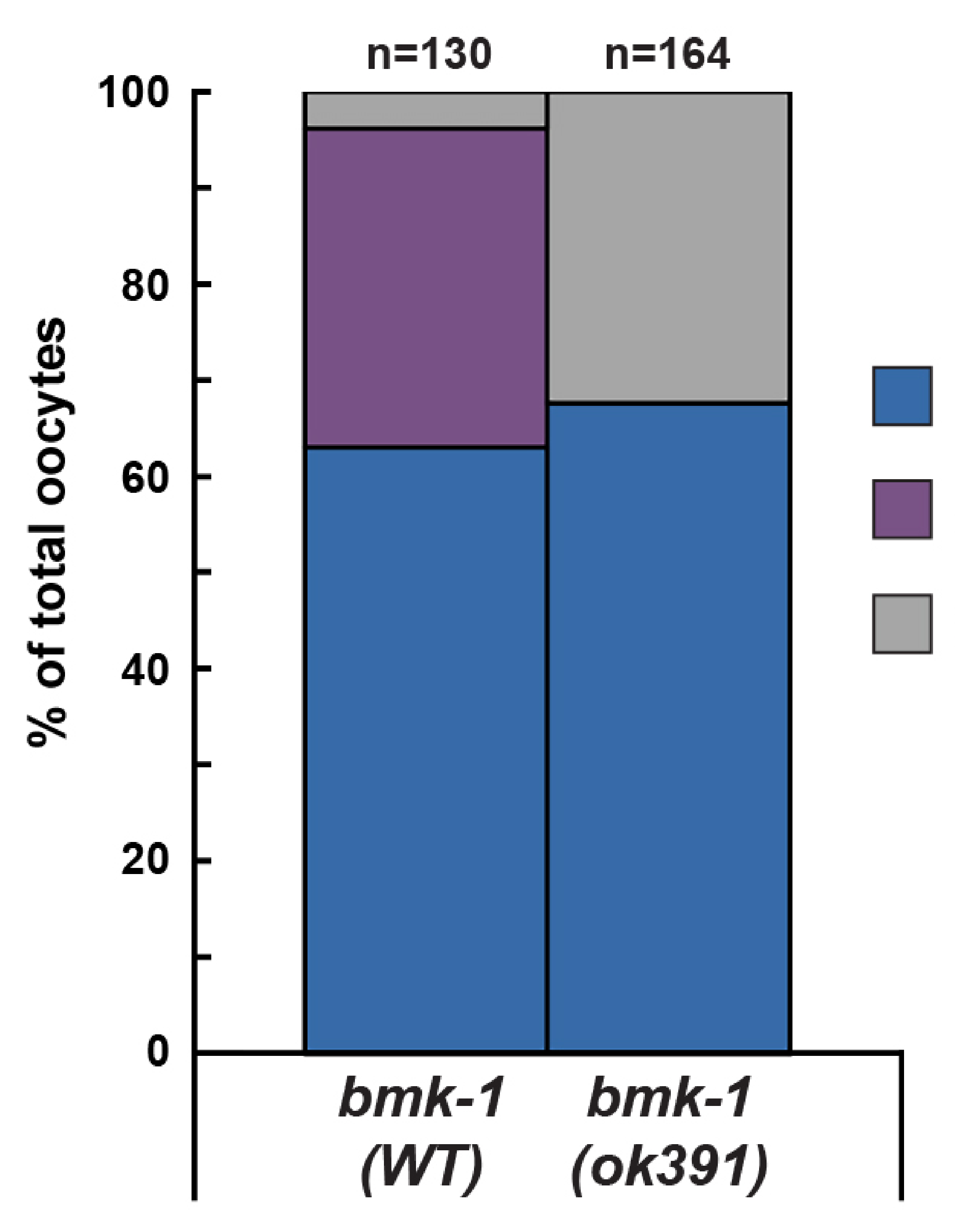

$\square$ No Segregation
k-1
391)
Monopole
breakdown
Mini
anaphase

\begin{tabular}{l} 
No Segregation \\
\hline k391) \\
Monopole \\
breakdown \\
Mini \\
anaphase
\end{tabular}

\begin{tabular}{l}
\hline No Segregation \\
\hline a1) \\
\hline Monopole \\
Meakdown
\end{tabular}

\begin{tabular}{l} 
No Segregation \\
\hline k-1 \\
391) \\
Monopole \\
breakdown \\
anaphase \\
Mini
\end{tabular}

breakdown

No

segregation
Monopole

GFP::tubulin, GFP::histone 
FIGURE 2 - figure supplement 1

A
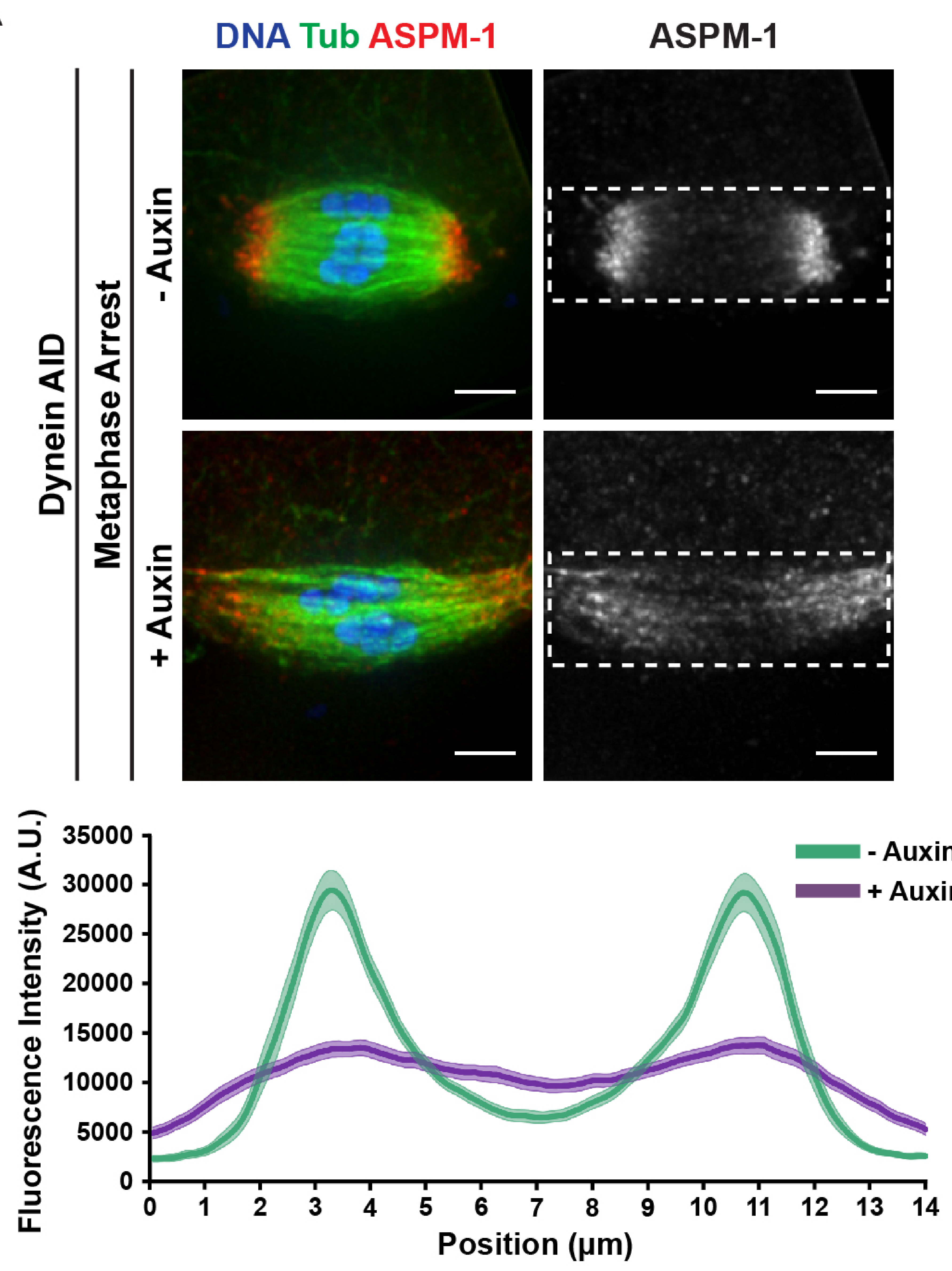

Arrested Spindle Length After-Auxin Treatment

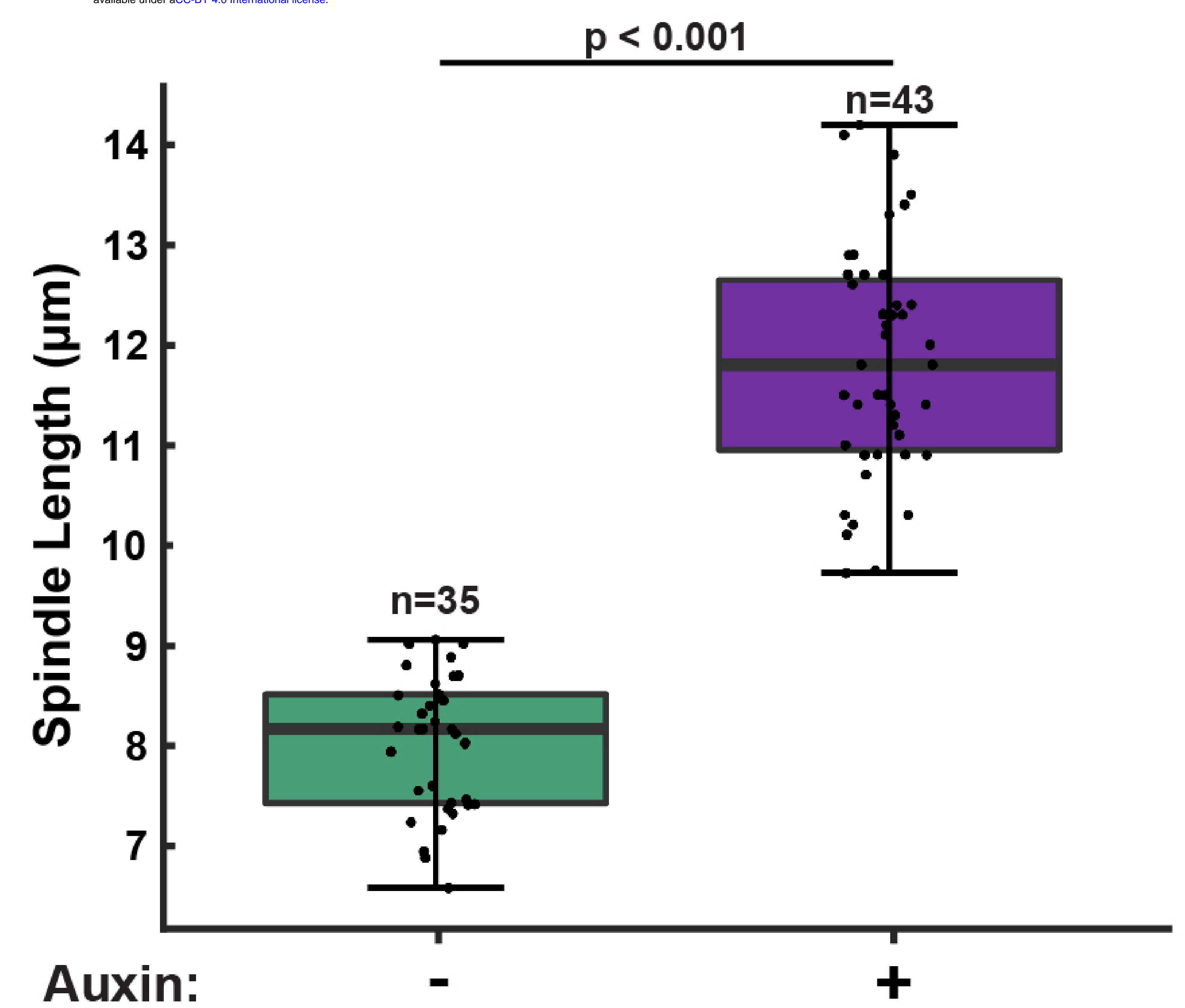

B
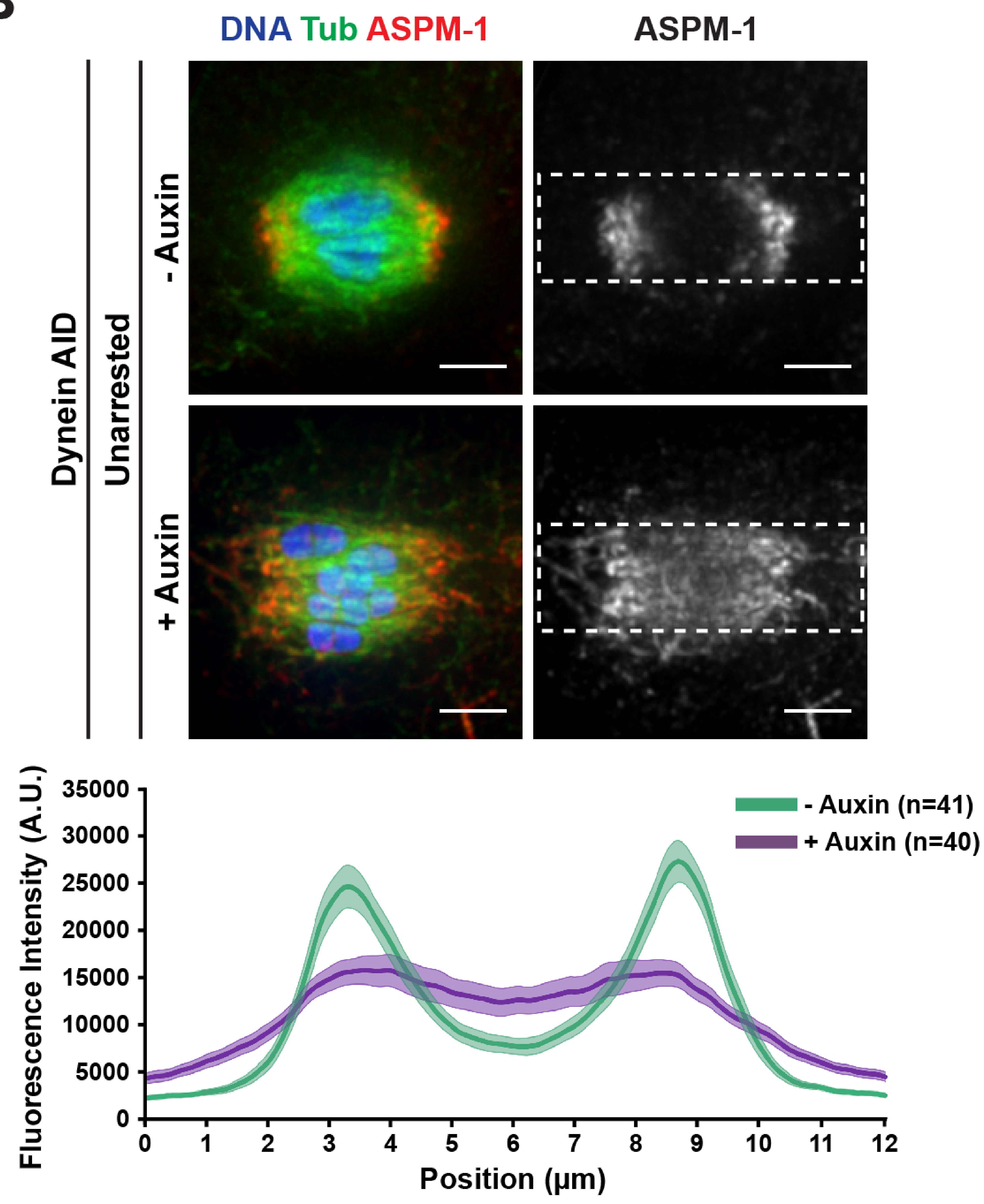

Unarrested Spindle Length After Auxin Treatment

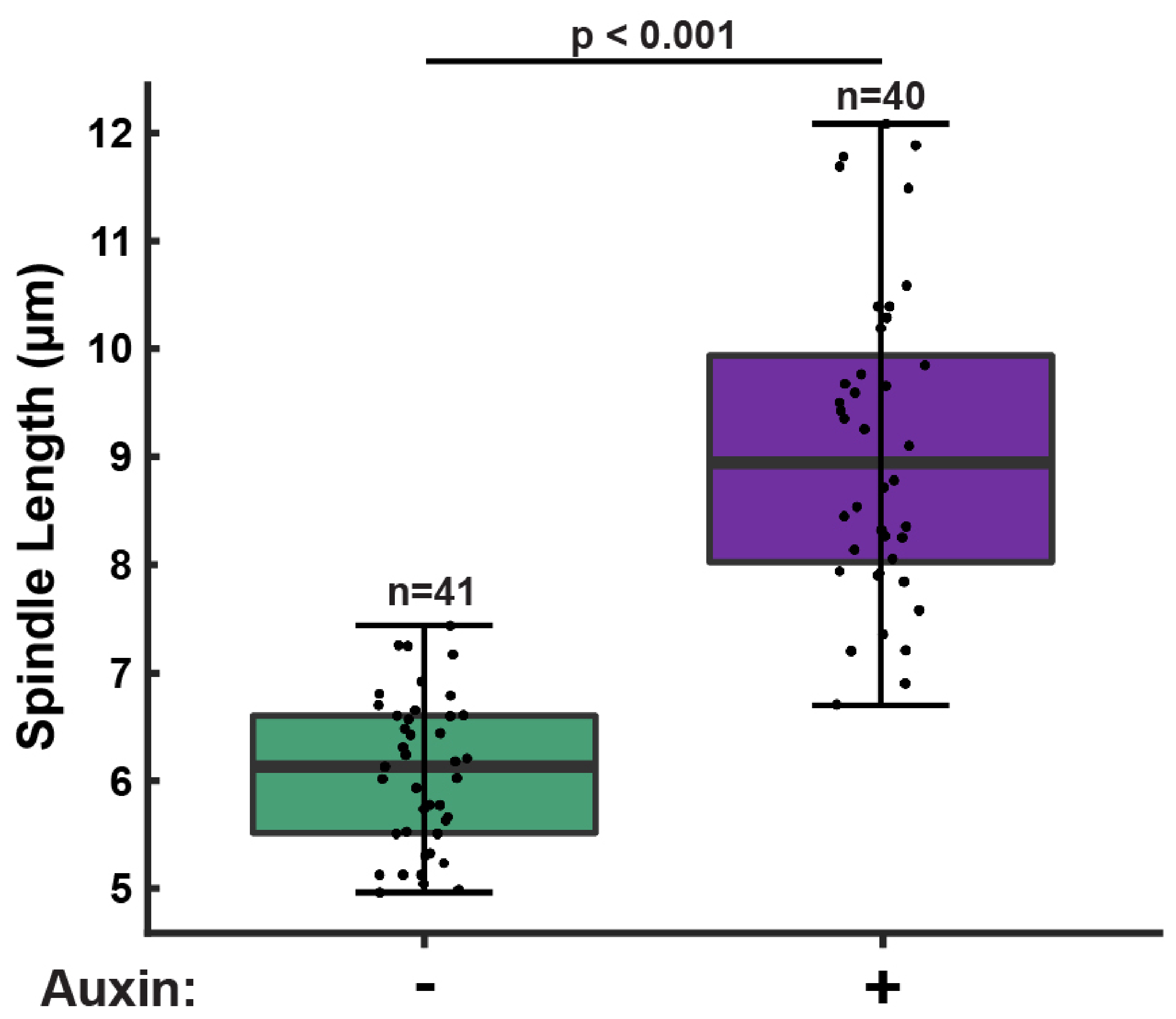


FIGURE 2 - figure supplement 2

GFP::tubulin; GFP::histone

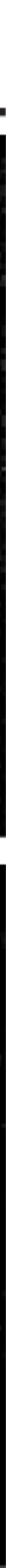


FIGURE 3 - figure supplement 1

A

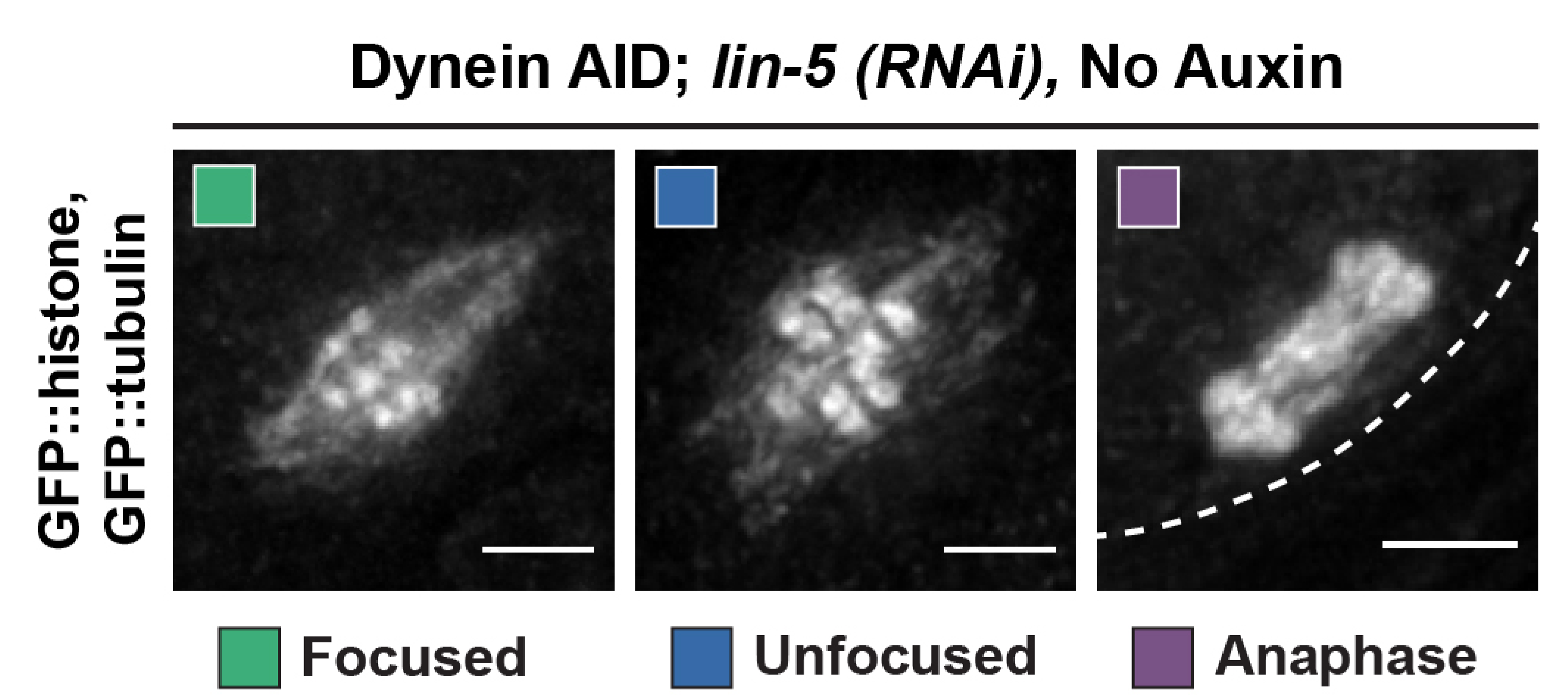

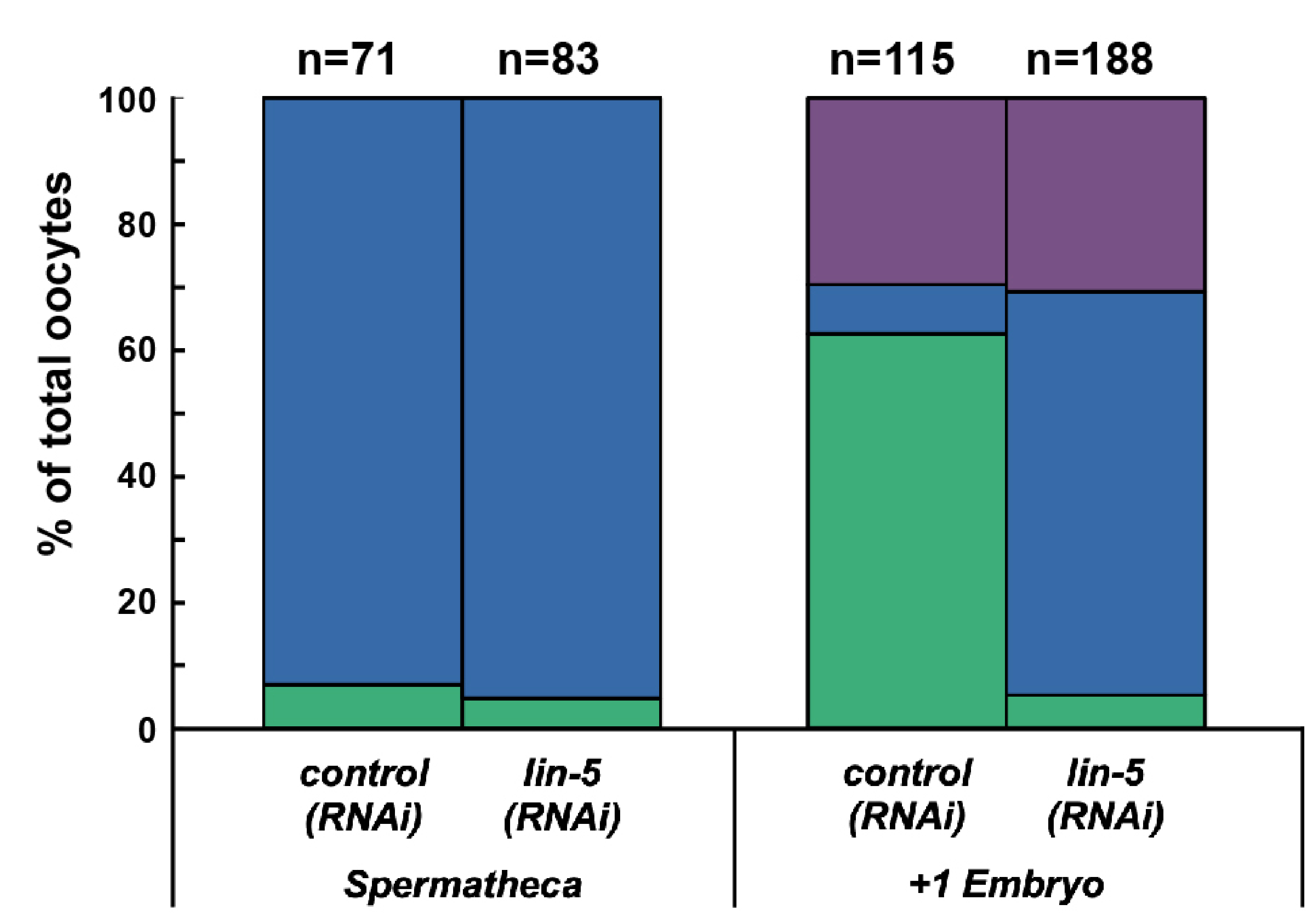

B

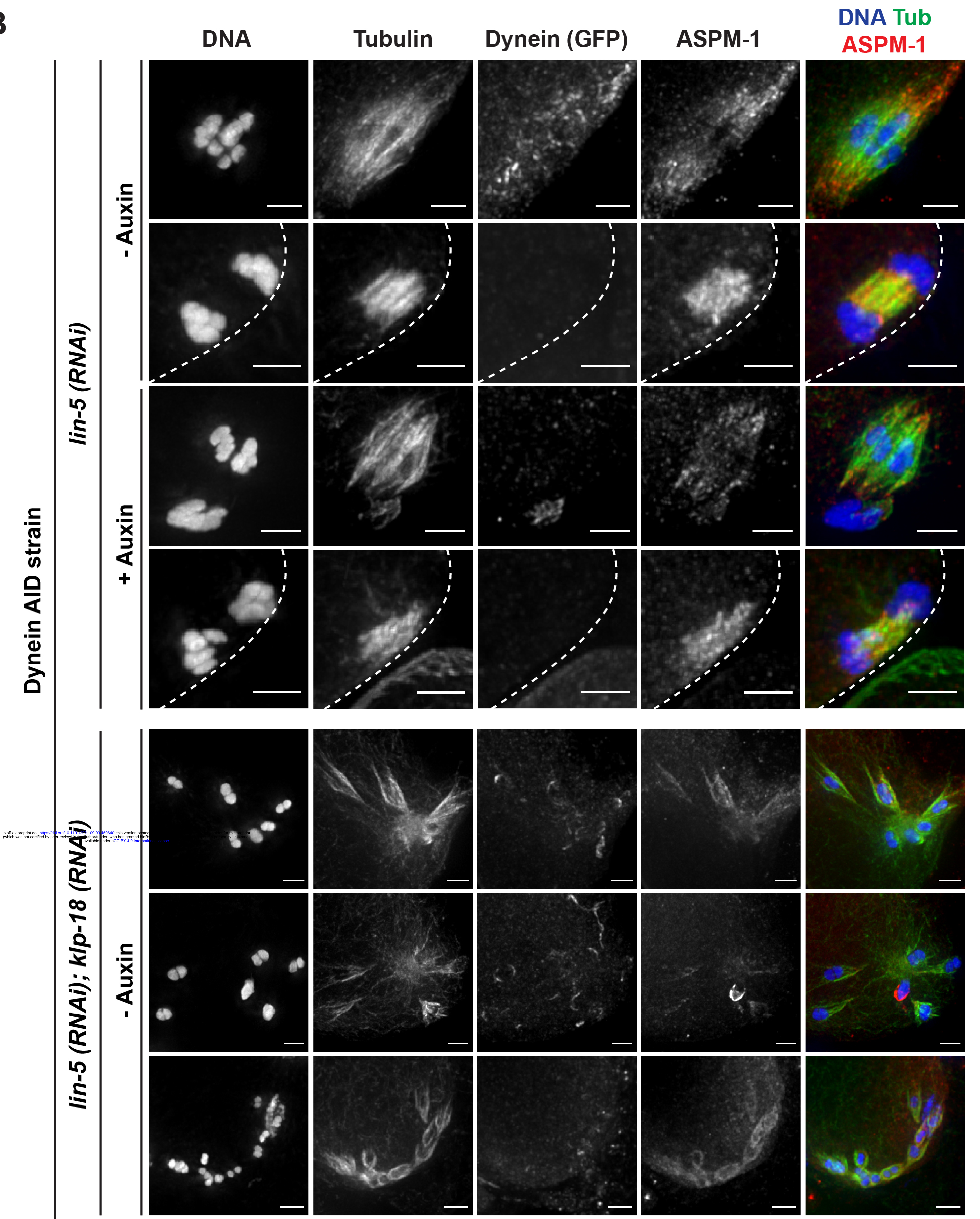




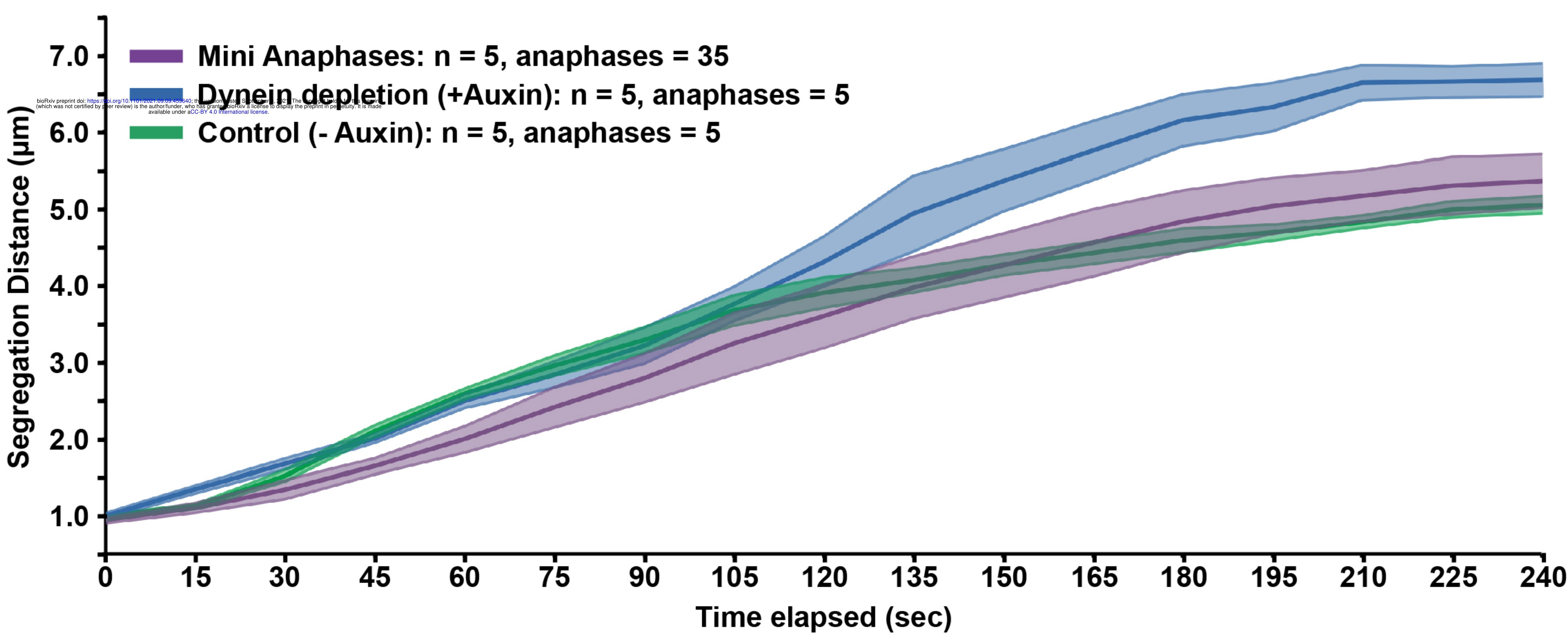


FIGURE 5 - figure supplement 1

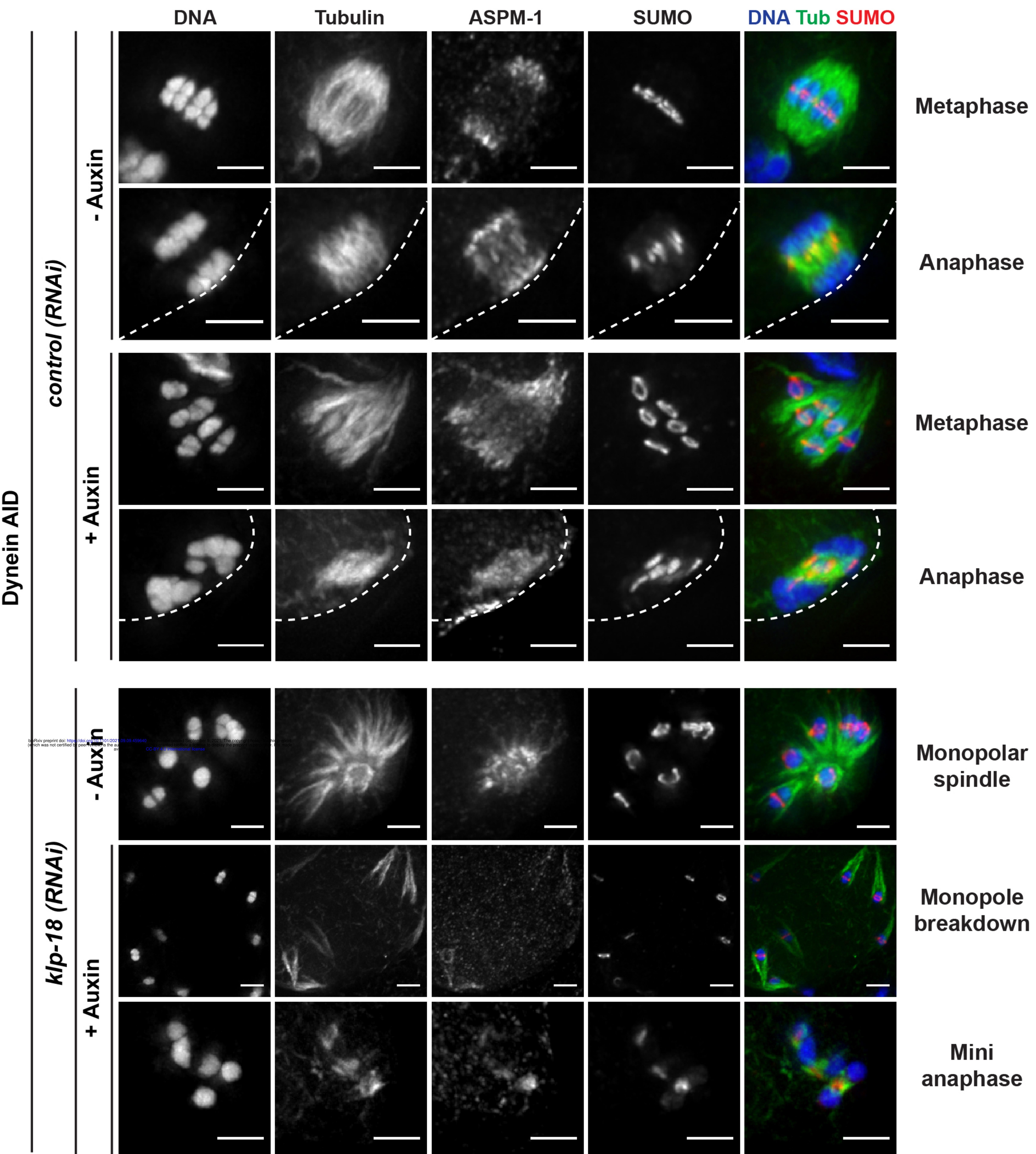


FIGURE 6 - figure supplement 1

A

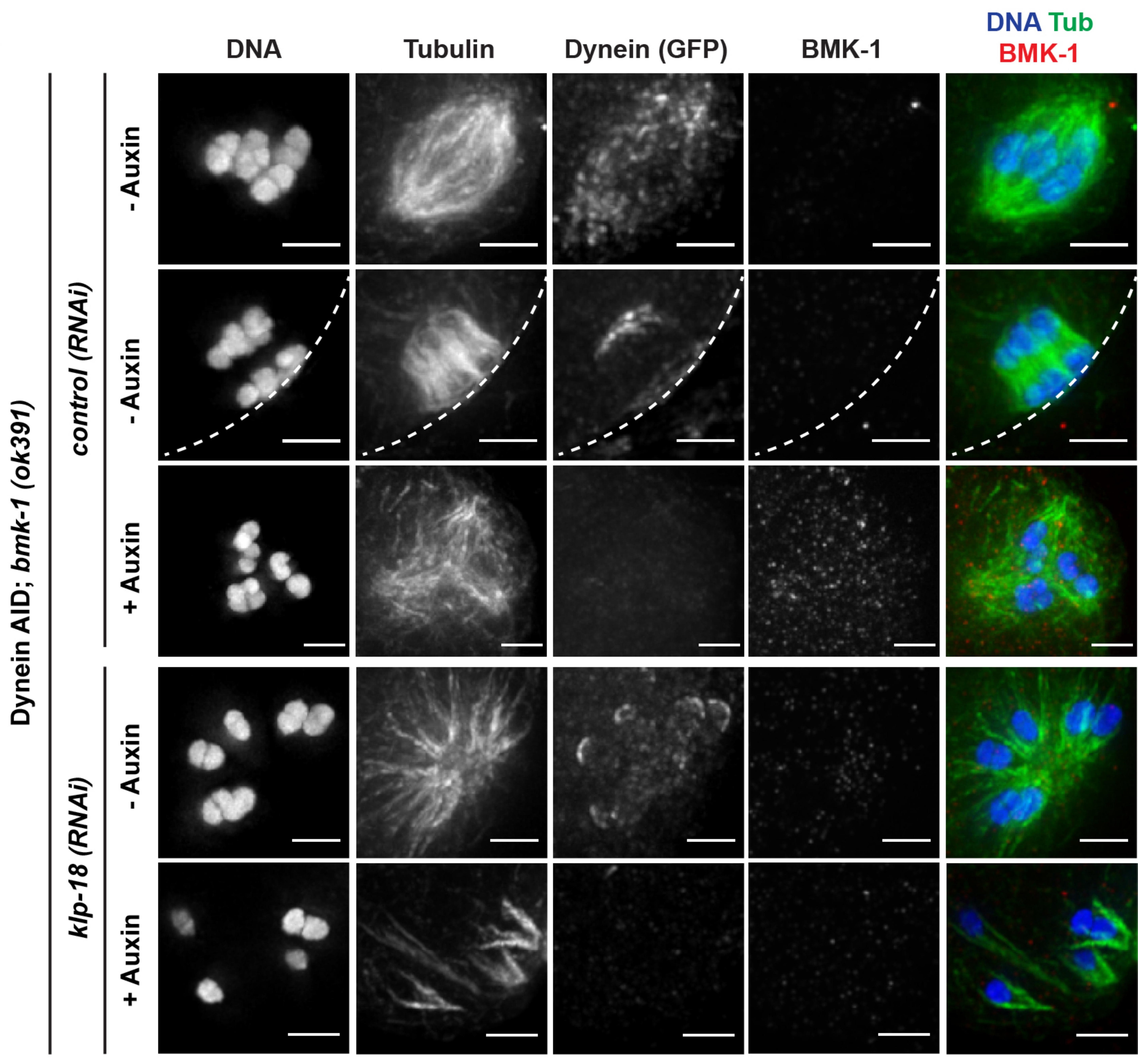

B

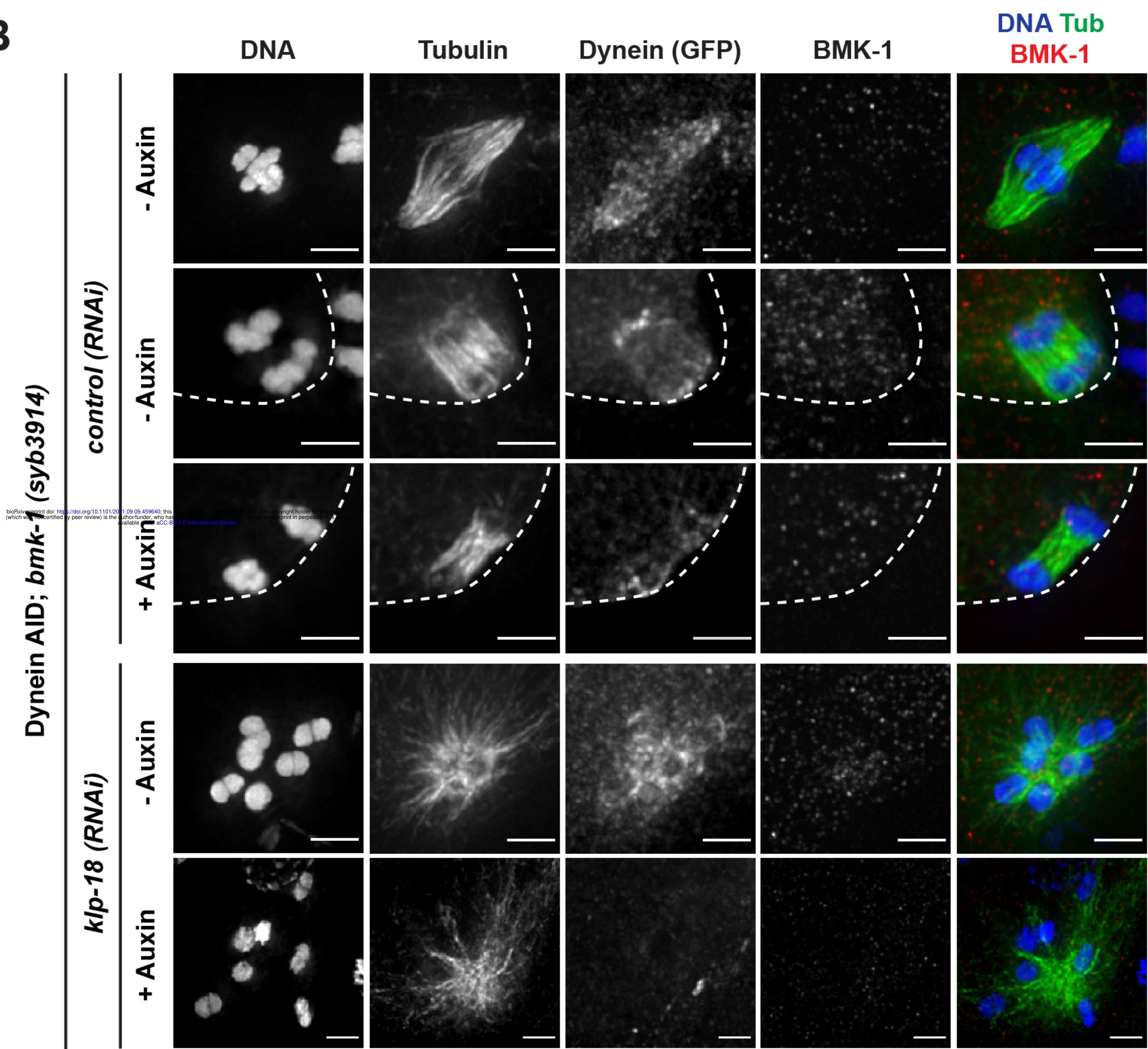

